\title{
PARTS OF THE BODY IN THE LATER GERMANIC DIALECTS
}

\author{
A DISSERTATION \\ SUBMITTED TO THE FACULTY \\ OF THE GRADUATE SCHOOL OF ARTS AND LITERATURE \\ IN CANDIDACY FOR THE DEGREE OF \\ DOCTOR OF PHILOSOPHY
}

DEPARTMENT OF GERMANIC LANGUAGES AND LITERATURES

BY

WILLIAM DENNY BASKETT

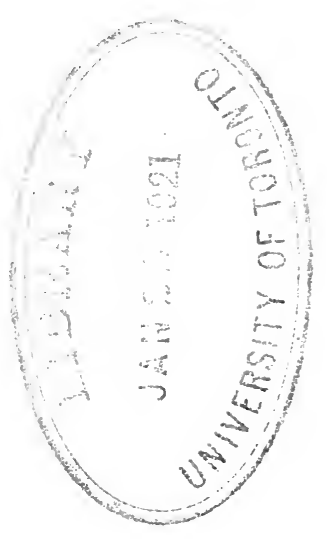

Linguistic Studies in Germanic, No. 5 THE UNIVERSITY OF CHICAGO PRESS

CHICAGO, ILLINOIS 
THE UNIVERSITY OF CHIOAGO PRESS

CHICAGO, ILLINOIS

THE BAKER \& TAYLOR COMPANY NEW YORE

THE CAMBRIDGE UNIVERSITY PRESS

LONDON

THE MARUZEN-KABUSHIKI-KAISHA

TOKYO, OBAKA, KYOTO, FUEUOKA, BENDAY

THE MISSION BOOK COMPANY

BHANGBA! 


\title{
Tbe Wniversity of Cbicago
}

\section{PARTS OF THE BODY IN THE LATER GERMANIC DIALECTS}

\author{
A DISSERTATION \\ SUBMITTED TO THE FACULTY \\ OF THE GRADUATE SCHOOL OF ARTS AND LITERATURE \\ IN CANDIDACY FOR THE DEGREE OF \\ DOCTOR OF PHILOSOPHY
}

DEPARTMENT OF GERMANIC LANGUAGES AND LITERATURES

BY

WILLIAM DENNY BASKETT

Linguistic Studies in Germanic, No. 5

THE UNIVERSITY OF CHICAGO PRESS

CHICAGO, ILLINOIS

I920 
Copyright $1920 \mathrm{BX}$

The University of Chicago

All Rights Reserved

Published February r920

Composed and Printed $\mathrm{By}$

The University of Chicago Press

Chicago, Illinois, U.S.A. 


\section{PREFACE}

This investigation deals with the words for the body and its parts in the later Germanic dialects. Its object is to show how these words came to have their present meaning, rather than to show the original meaning.

The method of procedure is to place beside each word under consideration a group of related words from the same dialect and from other dialects, in order to exhibit the primary meaning from which came the present meanings of the words in the group. Thus is shown the development of the use of a certain word as a name (usually a descriptive term) for a part of the body.

Those words for the body or its parts which were treated by Arnoldson (cf. Sources) have been omitted from this study. Frequent reference is made to his work.

The reader will observe often the abbreviation (W.) in the following pages. This will give some idea, but not a sufficient one, of the large aid given through inspiration and helpful criticism by Professor Francis A. Wood, of the University of Chicago. Quotations are made from his published works and unpublished notes. 



\section{SOURCES AND ABBREVIATIONS}

Arnl. = Arnoldson, Torild W. Parts of the Body in Older Germanic and Scandinavian. Chicago, 1915.

Bauer, Karl. Waldeckisches Wörterbuch. Herausgegeben von $\mathrm{H}$. Collitz. Norden und Leipzig, 1902.

Boekenoogen, G. J. De Zaansche Volkstaal. Leiden, 1897.

The Century Dictionary. New York, $1889 \mathrm{ff}$.

Cornelissen en Vervliet. Idioticon van het Antwerpsch Dialect.

Gent, 1899. Aanhangsel, 1906.

Crecilius, Wilh. Oberhessisches Wörterbuch. Darmstadt, 1897-99.

De Bo, L. L. Westvlaamsch Idioticon. Gent, 1892.

Diefenbach und Wülcker. Hoch- und Niederdeutsches Wörterbuch der mittleren und neueren Zeit. Basel, 1885.

Dijkstra, Waling. Friesch Woordenboek. Leeuwarden, 1900.

Draaijer, W. Woordenboekje van het Deventersch Dialekt. s'Gravenhage, 1896.

F. = Fischer, Hermann. Schwäbisches Wörterbuch. Tübingen, 1904-11.

Foll. $=$ Follmann, M. F. Wörterbuch der deutsch-lothringschen Mundarten. Leipzig, 1909.

Frisch. $=$ Frischbier, H. Preussisches Wörterbuch. Berlin, 1882-83.

Gallee, J. H. Woordenboek van het Geldersch-Overijselsch Dialect. s'Gravenhage, 1895.

Hertel, L. Thüringer Sprachschatz. Weimar, 1895.

Hintner, Val. Beiträge zur Tirolischen Dialektforschung. Der Defregger Dialekt. Wien, 1878.

Hoenig, Fritz. Woerterbuch der Koelner Mundart. Koeln, 1905.

Hyrtl, Joseph. Die alten deutschen Kunstworte der Anatomie. Wien, 1884.

Jam.=Jamieson. Scotch Dialect Dictionary. Edinburgh, 1806; Supplement, 1826.

Jongeneel, J. Een Zuid-Limburgsch Taaleigen. Proeve van Vormenleer en Woordenboek der Dorpspraak van Heerle. Heerlen, 1884.

Kisch, Gustav. Vergleichendes Wörterbuch der Nösner und moselfränkisch-luxemburgischen Mundart. Hermannstadt, 1905. 
Koolman, J. ten Doornkaat. Wörterbuch der Ostfriesischen Sprache. Norden, 1879-84.

Leihener, E. Cronenberger Wörterbuch. Marburg, 1908.

Leuvensche Bijdragen op het Gebied van de Germaansche Philologie, etc. Antwerpen, s'Gravenhage, Leipzig, $1896 \mathrm{ff}$.

Lexer, Matthias. Kärntisches Wörterbuch. Leipzig, 1862.

Lübben, August. Mittelniederdeutsches Handwörterbuch, vollendet von Christoph Walter. Norden und Leipzig, 1888.

Lux. $=$ Wörterbuch der Luxemburgischen Mundart. Luxemburg, 1906. Martin und Lienhart. Wörterbuch der elsässischen Mundarten. Strassburg, 1899-1907.

Meisinger, O. Wörterbuch der Rappenauer Mundart. Dortmund, 1906.

Molema, H. Wörterbuch der Groningschen Mundart im neunzehnten Jahrhundert. Norden und Leipzig, 1888.

Müller-Fraureuth, K. Wörterbuch der Obersächsischen und erzgebirgischen Mundarten. Dresden, 1914.

Neubauer, Joh. Bezeichnungen des menschlichen Körpers und seiner Theile im Egerlande. Bayerns Mundarten, Band II, Heft II. München, 1894.

Regel, Karl. Die Ruhlauer Mundart. Weimar, 1868.

Schm. =Schmeller, J. A. Bayrisches Wörterbuch. München, 1872.

Schmidt-Petersen, J. Wörterbuch und Sprachlehre der nordfriesischen Sprache. Husum, 1912.

Schu.$=$ Schuermanns, L. W. Algemeen Vlaamsch Idioticon . Leuven, 1865-70; Bijvoegsel, Loven, 1883.

Schullerus. Siebenburgisch-sächsisches Wörterbuch. Strassburg, $1908 \mathrm{f}$.

Schultze, M. Idioticon der Nord-Thüringischen Mundart, Nordhausen, 1874.

Schw. = Schweizerisches Idioticon. Wörterbuch der schweizerdeutschen Sprache. Frauenfeld, 1881-.

Sipma, P. Phonology and Grammar of Modern West Frisisn. Oxford University Press, 1913.

Stratmann, F. H. A Middle English Dictionary, revised and enlarged by Henry Bradley. Oxford, 1891.

Teierlinck, Is. Zuid.-Oostvlaandersch Idioticon. Gent, 1908. 
Tuerlinckx, J. F. Bijdrage tot een Hagerlandsch Idioticon. Gent, 1886.

U-K. = Unger-Khull. Steirischer Wortschatz. Graz, 1903.

Van Dale. Groot Woordenboek der Nederlandsche Taal. s'Gravenhage en Leiden, 1898.

Verdam, J. Middelnederlandsch Handwoordenboek. s'Gravenhage, 1911.

Vilmar, A. F. C. Idioticon von Kurhessen. Marburg und Leipzig, 1868. Nachträge, Hermann von Pfister. Marburg, 1886.

Weinhold, Karl. Beiträge zu einem sch́lesischen Wörterbuche. Wien, 1855.

Wg. = Weigand, Fr. L. K. Deutsches Wörterbuch. Giessen, 1909-10.

Wo. $=$ Woeste, Fr. Wörterbuch der Westfälischen Mundart. Norden und Leipzig, 1882.

Wr. $=$ Wright, Joseph. The English Dialect Dictionary. London, 1898-1905. 


\section{Digitized by the Internet Archive in 2007 with funding from Microsoft Corporation}




\section{CONTENTS}

Parts of the Body

1. Body

2. Head-3. Baldhead-4. Forehead-5. Hair-6. Face-7. Mouth8. Lip-9. Nose, Snout, Beak-10. Nostril-13. Chin-14. Double Chin, Dewlap, Wattle-15. Cheek-16. Gill-17. Jaw-18. Gums-19. Tooth20. Enamel-21. Palate-22. Tongue-23. Tonsil-24. Uvula-25. Ear -26. Eye-27. Pupil-28. Eye Socket-29. Eyelash-30. Eyebrow31. Temple-32. Crown-33. Parting of the Hair-34. Crest-35. Beard -36. Mane-37. Skull-38. Fontanelle-39. Brain

40. Neck-40a. Withers-41. Throat-42. Larynx-43. Windpipe44. Gullet

45. Shoulder-46. Shoulder Blade-47. Arm-48. Elbow-49. Wrist -50. Hand, Fist-51. Palm-52. Paw-53. Finger-54. Thumb55. Forefinger-56. Middle Finger-58. Little Finger-59. Nail-60. Claw -61 . Wing-62. Feather-63. Fin-64. Flipper

65. Crotch-66. Groin

67. Limb-68. Thigh-69. Leg-70. Calf-71. Knee-72. Kneepan73. Bend of the Knee-74. Foot-75. Sole-76. Instep-77. Ankle78. Heel-79. Hoof-80. Toe

81. Breast, Udder, Teat-82. Bosom-83. Thorax-84. Waist85. Belly-86. Pit of the Stomach-87. Navel-88. Umbilical Cord

89. Back-90. Buttocks-91. Anus-92. Tail-93. Flank, Side94. Loin-95. Lap

96. Heart-97. Pericardium-98. Valve of the Heart-99. Lung100. Kidney-101. Crop-102. Gall Bladder-103. Gizzard-104. Stomach -105. Omasum-106. Abomasum-107. Peritoneum-108. Pleura109. Gland-110. Pancreas-111. Thymus-112. Diaphragm-113. Intestines-114. Rectum-115. Viscera-116. Appendix-117. Bladder118. Bladder Duct-119. Womb-120. Ovary-121. Oviduct-122. Genitals, Pudenda-123. Penis-124. Prepuce-125. Scrotum-126. Testicle -127. Pudendum Femininum-128. Hymen-129. Afterbirth-130. Caul -131. Membrane

132. Skin-133. Epidermis-134. Scale, Shell-135. Pore-136. Tendon-138. Muscle-139. Blood-140. Flesh, Fat-141. Flesh (to eat)142. Bacon-144. Quick 
xii

CONTENTS

145. Horn-146. Antler-147. Antenna-148. Sting-149. Joint150. Cartilage

151. Bone-152. Frontal Bone-153. Malar Bone-154. Jawbone155. Ethmoid Bone-156. Incus-157. Hyoid Bone-158. Spine159. Spinal Cord-160. Vertebra-161. Breastbone-162. Clavicle163. Long Bones of the Arm-164. Carpus-165. Phalanges-166. Knucklebone-167. Hip Bone-168. Femur-169. Shank Bone-170. Splint Bone-171. Tail Bone-172. Fishbone-173. Periosteum-174. Rib175. Skeleton-176. Quarter

INDEX . . . . . . . . . . . . . 137 


\section{PARTS OF THE BODY}

[An alphabetical index may be found at the close of the volume.]

\section{BoDY}

The body is described (A) as a bulging, big object or a trunk; (B) as a container, such as a sack, basket, cart; (C) as a covering; (D) as the form or shape of persons; (E) as the fleshy part; (F) as something naked; $(G)$ as the podex.

1A1. NFris. bent Rumpf, Körper; Schmiele, OFris. bend, band Band, OE. byndel bundle.

1A2. Bav. bulgen Sack, etc.; der hohle Leib, bulken Leib, MDu. bulge swelling, NE. bulge, bilge protuberant part of a cask or ship. Cf. 113A2.

1A3. Steir. büttling Körper mit Ausnahme des Kopfes; runde, grössere Pflaumengattung, Kärnt. junges Kalb, NHG. bütte Daubengefäss.

1A4. MDu. stronc trunk; trunk of the body, NHG. strunk kurzer, dicker Stengel.

1A5. Bav. garbm Leib, garbe Stück Fleisch, NHG. garbe.

1A6. MLG. krop Rumpf, Leiche; Kropf, runder Auswuchs; Schlund. Compare Arnl. 127:03.

1A7. EDial. croopin body, trunk of a man or an animal, NE. croope, croup rump, Fr. croupe croup, loan-word from Germ. Cf. 1A6.

1A8. Du. raap turnip, rape; body, NE. rape.

1B1. Fris. aeikoer body. Cf. 90A42.

1B2. EDial. applecart the human body.

1B3. Swiss sack Sack; menschl. Leib, madensack Leib. Cf. $85 \mathrm{~A} 46$.

1B4. NFris. sjuarenpös menschl. Körper, sjuaren Scheisse +pös Sack, MHG. pfose Beutel.

1C1. Fris. bast cortex; corpus, properly skin, DuDial. baste skin, body. Cf. 132A1.

1C2. MDu. vacht skin, hide; body, OHG. fahs, OE. feax hair. Cf. Arnl. 162:49. 
1C3. DuDial. hoed, hud body, Du. huid hide. Compare MDu. huut ende haer, NE. hide and hair, the whole person.

1D1. DuDial. leest body, Du. form (of the body), NHG. leisten.

1E1. EDial. carcase human body, trunk, OFr. carcas skeleton, frame.

1E2. MDu. corne body, Lat. caro, carnem.

1F1. Swiss blutz nackter Leib, NHG. blutt bloss. Cf. 3B3.

1G1. Du. gat aarsgat; body. Cf. 91A3.

1G2. DuDial. donder body. Abstracted from donderment body, a blend of Du. donder thunder and fondament foundation; bottom, buttocks.

\section{HEAD}

The head is described (A) as a ball, knob, knot, nut, chunk, block, and the like; (B) as a swelling; (C) as a bushy top, bush, tuft; (D) as a hood or turban; (E) as the highest part, top; (F) as a container, such as a chest, box, chamber; $(G)$ as a covering, such as a roof or lid; $(\mathrm{H})$ as a sherd, in reference to the skull, or as the skull; (I) as scurf; (J) as that which strikes, pushes, butts, nudges; (K) as that which hunts about noisily; (L) as that which sways, nods, bobs; (M) as the brain, which is the seat of the understanding or which is a soft pulpy mass; $(\mathrm{N})$ by a proper name; $(\mathrm{O})$ in terms of contempt; (P) as a hairy slope; (Q) unclassified.

2A1. Swab. aeppel Apfel; Kopf des Menschen.

2A2. EDial. jobber-nowl head; dunce, blockhead, app. from jobbard stupid fellow + noll, OE. hnol head (NED.).

2A3. Fris. bol head, Du. idem.; ball, MDu. bolle idem.; trunk of a tree, NE. boll. Cf. $26 \mathrm{E} 1$.

2A3a. NE. block head.

2A4. Swiss bluwel schwerer Holzschlegel; grosser, dicker Kopf; etwas Schweres, Plumpes, OHG. bluwil Nomen instr. zu bliuwan schlagen.

2A5. Steir. plutzer Krug; Kürbis; Kopf, Kärnt. plutzar grosser Kürbis; grosser Kopf, Schles. plutzer breite Kartoffel, Swab. blutzer Kürbis.

2A6. Swiss potsch Kugel; Baumnuss; Kopf, NHG. butschen stossen, butsch Stoss. 
2A7. DuDial. pruikebol head with hair, Du. idem; wooden head on which wigs are made, pruik wig. Sax. parabel Kopf is probably a corruption of the same word. Cf. $2 \mathrm{~A} 3$.

2A8. MLG., MDu. pol(le), LG. pull Kopf; Wipfel, NE. poll head, NHG. haarpull Kopf, pull Art Knaul, EFris. pol rund, voll, ON. pollr rundliche Meerbucht.

2A9. DuDial. fritus, frietus, friet head, Du. frit molten mass of glass, Fr. fritte molten glass.

2A9a. NE. topknot head. Cf. 5A23.

2A10. EDial. tatie potato; head.

2A12. Als. tschutter Kopf, NHG. schotter einzelne Blöcke, schutt hingeschütteter Überrest von Erde, Steinen.

2A13. EDial. sow sow; head; stack, heap, verb to stack.

2A14. Swiss chabis, Swab. kabes cabbage; head, NE. cabbage idem. Cf. 81B16.

2A15. MLG. kolve Kopf mit kurz gestutztem Haar, NHG. kolbe idem; Kopf, Ruhl. kålwen Kolbe; Kopf, Zwickauer khulwe Kopf, OHG. kolbo Kolben.

2A16. Sax. kohlrabi Kohlart; Kopf.

2A17. EDial. conk head. Cf. 9A15.

2A18. EDial. costard kind of apple; head. Cf. 2 A19.

2A19. EDial. costrel keg, barrel; head, OFr. costerel winecask (Wr.). Cf. preceding.

2A20. EDial. chump block, stump; head, NE. chunk; thick, blunt end. Cf. 40A7.

2A21. Swiss chrübis Kopf; Knirps. Blend of chr(ips)+ (ch) $\ddot{u}(r) b i s . \quad$ Cf. 2A22, 2 A23.

2A22. Swiss chürbs Kürbis; Kopf. Cf. preceding.

2A23. Swiss chnips Kopf. Blend of Swab. kn(ilps) fish with large head (Swiss chnolpen chunk, lump) $+($ chr $)$ ips larynx. Cf. $2 \mathrm{~A} 21$.

2A24. Lux. klatz Kugel; Apfelkrapfen; Kopf, Schädel, NHG. klotz fest zusammenhangende unförmliche Masse, MHG. kloz klumpige Masse.

2A25. Als. krabutz Kopf; $p l$. kleine Kinder, from $k r a b(b e)$ regsames muntres kleines Tier+Als. (kr)utze Kerngehäuse des Obstes; Knirps. Wood, JEGPh., XI, 310. 
2A26. EDial. knaup hillock; head; lump, swelling, NHG. knauf.

2A27. EDial. knob lump, bump; low roundish hill; head. Cf. 9A10, 80A1.

2A28. EDial. knoll small hill; head; lump, OE. cnoll hilltop, cop, etc.

2A29. EDial. knot lump; bud of a plant; top of the head. Cf. $166 \mathrm{~A} 1,40 \mathrm{~A} 8$.

2A30. EDial. knurr knot of wood; ball; head, NHG. knorren.

2A31. Kärnt. laus-kugel Kopf.

2A32. Sax., Als., Swiss rübe Rübe; Kopf, OHG. ruoba Rübe. Cf. 92B5a.

2A33. Swab. marbel Marmor; Steinkügelchen; Kopf.

2A34. Swab. maser knorriger Auswuchs an Bäumen; Pfeifenkopf; Kopf, OHG. masar knorriger Auswuchs an Bäumen.

2A35. DuDial. mörk head, MLG. morke Rübe. Cf. 7H18.

2A36. Swiss mutsch Brotlaibchen; dicker Kopf, Als. mutschel idem, Swiss mutsch stumpf; grob, dick, MHG. mutsche Brotlaibchen. Cf. $126 \mathrm{~A} 17$.

2A37. Swiss näggel unentwickelte Kirsche, Erdbeere; Saugzapfen; Kopf. Cf. 2 A40.

2A38. EDial. nap, nappy vessel, kettle; head, OE. hnaep cup, bowl.

2A39. Sax. nips pea, bean, apple seed, plum pit, small ball; head.

2A40. Swiss niggel Holzklotz; Knicker; Kopf, Prus. nickel kleines Pferd.

2A41. NE. noggin wooden vessel; head, Gael. noigean wooden cup, cnag knob, etc. (CD).

2A42. Swiss nuss, NE. nut, coco-nut head. Cf. 127A30, $126 \mathrm{~A} 18$.

2B1. Als. barpel Blase auf der Haut; Impfpustel; dicker Kopf. MHG. barpel variola.

2B2. Als. bibele, Swiss bibeli, bibi Hitzbläschen; grosser Kopf. Cf. 6E1, 85A5, $101 \mathrm{~A} 1$.

2B3. EDial. pimple head, NE. pimple.

2B4. Als. bulti Spielkugel; dicker Kopf. Cf. 85A16, 89A2, $104 \mathrm{~A} 2$.

2B5. Als. burzel Kopf. Cf. 90A19. 
2B6. NHG. dusel Beule; Kopf, duseln schlagen, stossen.

2B7. Thur. tössel Kopf, MLG. dössen blähen, bauschen.

2B8. EDial. crop head. Cf. 104A6.

2B9. EDial. crummy head, NE. adj. plump, full-figured, crumb inner part of a loaf; plumpness.

2B10. Swiss gutsch kleiner, rundlicher Hügel; Felskopf; Kopf.

2B11. Bav. hübel Hügel; Beule; Kopf, MHG. hübel Hügel.

2B12. Swïss mauschen grosser Kopf, Als. mäusche Kopf; Prügel, Swiss muscheli etwas Volles, Rundes, Weiches. Cf. 113A5.

2C1. Sax. pinsel Kopf, NHG. Haarbüschel am Stiel, OFr. pincel Pinsel. Cf. 123C10.

2C2. Swab. dolder Sprossende einer Pflanze; Wipfel eines Baumes; Kopf, OHG. toldo Wipfel eines Baumes. Cf. 5A17. $5 \mathrm{~A} 18$.

2C3. Als. döller grosser Ast; Gipfel des Baumes; Kopf. Cf.

2C4. Wald. dülts Kopf. Cf. 2C2.

2C5. Als. tupé in die Höhe gestelltes Stirnhaar; Kopf, Fr. toupet tuft of hair, etc.

2C6. Sax. schiebe Kopf; Dickkopf, MHG. schoup, NHG. schaub Strohbund, -wisch. Cf. 5A24.

2C7. Swiss grotzen buschiges Tännchen; Wipfel; Kopf, Swab. Sprosse.

2C8. DuDial. kopstubber mop; head with long bristly hair, EFris. kopstübber head-shaped broom.

2C9. Swiss hürsel Kopf. Für *hürschel zu hürschen verwirren, gehürsch Wirrwarr, ungeordnetes Haar (Schw.).

2C10. Als. hurst Busch; Kopf, MHG., OHG. hurst Gesträuch, Dickicht.

2C11. Als. nest Nest; Hinterkopf. Cf. 5A42.

2D1. Swiss beginen Häubchen; Kopf, Fr. beguin Nonnenhäubchen (Schw.).

2D2. Als. domino Kopf; eine Art Haube.

2D3. Sax. tulpe Kopf, Du. tulband turban, Turkish tulbend idem (Franck), Kilian tulpe tjara.

2D4. Kärnt. guffe Hut, Kopf, OHG. kuppa mitra, MHG. gupfe idem.

2D5. Als. gugard Kopf, Fr. cocarde cockade; hat (M and L). 
2D6. Swiss hübis Kopf, hube Haube+verächtl. -iss (Schw.).

2E1. Lux. fischt Dachfirste; Kopf; Vorderteil, OHG. first Gipfel, Dachgiebel.

2E2. EDial. tip head; peak of a cap.

2E3. MLG. top Zopf; Spitze; Kopf, MDu. top of the head, NFris. toop, top Stirn, Scheitel; Zopf, NE. top tuft, crest on the apex of anything; hair of the head; crown of the head, sinciput, EDial. topping head, OE. topp top, summit; lock of hair, OHG. zopf Haarflechte; Ende eines Dinges.

2E4. Swiss, Swab. girbel head. From $g(i p f e l)+(w)$ irbel. Cf. $32 \mathrm{~A} 5$.

2E5. EDial. capper head, cap master, chief, verb put a covering over a sheaf of corn, crown a wall with mortar.

2E6. Als. kipp Gipfel des Baumes; Dachfirst, Kopf, Obersächs. kippe Höhe; Spitze, NHG. kippe.

2E7. Fris. kopstik head, kop head, stik piece, part.

2E8. NE. coxcomb comb of a cock; top of the head, head.

$2 \mathrm{~F} 1$. Steir. pavesenkammer Schädel, Kopf, pavesen sandwich with brains.

2F2. Als. tabernakel Heiligtum; Kopf.

2F3. Cologne gedankenschaaf Kopf, schaaf Schrank.

2F4. Prus. grützkasten Kopf, grütz Verstand, NHG. grütze brains.

2F5. NE. knowledge-box head.

2F6. MDu. mole mold; head, brain pan, OFr. mole matrix, mold.

2G1. Cologne daach Dach; Kopf, Prus., Oberh. dach idem. Cf. 89B1.

2G2. Lothr., Als. deckel Deckel; Kopf, Sax. strohdeckel idem.

2G3. EDial. dicky head, skull, NE. a bib, dick leather apron, Du. dek a covering (NED.).

2G4. EDial. scalp head, skull, ME. top of the head, scalp, Du., schelp shell, ON. skalpr, Swed. skalp sheath, DanDial. skalp hull. Compare Wood, Hesperia, Ergänzungsreihe, I, $62 \mathrm{f}$.

2G5. Fris. holle head, DuDial. hul woman's cap; head, Hess. holle bird's crest, MDu. hulle cap; hull, shell; covering, MLG. Kopfbedeckung, OHG. helan conceal. Cf. 5A38, 34B2.

2H1. Westf. scherwe, Lux. schirbel Scherbe; Kopf, testa, OHG. scirbi Scherbe. 
2H2. Lothr. schirmel Scherbe, Schale; Kopf, schirme Scherbe, Als. schirmen idem; irdnes Gefäss. Cf. preceding.

2H3. Bav. schädel for head.

2I1. Als. micker head, mickes scurvy head; scurf, Swiss miggiss Gemenge; Unrat, Kehricht; scurf. Compare similar use of OHG. grind, Arnl. 1:51.

2I2. Als. miges Kopf. Cf. $2 \mathrm{I} 1$.

2J1. EDial. bash front of the head of a bull or pig; dint caused by a blow, verb beat, crush.

2J2. Fris. peut head, peuter a blow, DuDial. poot head, poten to set in the ground, plant, OE. potian push, thrust, butt.

2J3. EDial. nudger head, nudge a slight push, verb move.

2K1. Swiss, Sax. nüschel Kopf, Schles., Sax. nischel idem, Swiss nuschen mit einem raschelnden Geräusch in etwas herumwühlen, Thur. nüscheln schnüffeln, lecken, schmatzen. Cf. 5E12, 9L11, $7 \mathrm{C} 9$.

2L1. Prus. dämel Kopf, dämeln dumm tun, tändeln, Livland schaukeln, NHG. schlaftrunken sein; herumschlendern. Wg. compares Skt. tamyati betäubt werden.

2L2. Sax. gimms Kopf, Swiss gimslen sich hin und her bewegen.

2L3. EDial. niddick back of the head; head; skull, noddle head, ME. nodile head, nape of the neck, EDial. noddle make light and frequent nods, NE. nod. Wood, Hesperia, Ergänzungsreihe, I, 33. Cf. $40 \mathrm{G} 1$.

2M1. Steir. hiren brain; brow; head, Als. hirni idem. Cf. 4D1.

2M2. EDial. pash brains; head; rotten or pulpy mass; mud and slush, pashhead idiot, soft-headed person. Cf. 39A1.

2N1. EDial. nancy effeminate man; head; the pudding in a pig which is next in size to the paunch.

201. EDial. two-penny cheap; contemptible, subst. weak ale, etc.; head.

202. Swab. laüs-markt Kopf.

203. Sax. rotz-batterie Kopf.

2P1. Swiss g'hor halde (haar-halde) Kopf.

2Q1. Sax. dammich einer der viel flucht; Feldwebel; Hinterkopf: Kürzung aus Gott verdamme mich. Er hat den Hut auf dem Dammich =Der Hut sitzt schief. Cf. 2Q2. 
2Q2. Als. scharnippel Hinterkopf, scharnippeldekoton leichter Fluch. Er hat den Hut scharniple auf dem Ohre sitzen. Cf. 2 Q1.

2Q3. NE. pate head, more particularly applied to the part usually covered with hair; skin of a calf's head. Origin unknown (NED.).

\section{BALDHEAD}

The bald head is described (A) as something flat; (B) as a gleaming object.

3A1. Steir. tanzboden Tanzplatz; Glatze, kahler Kopf.

3A2. Swab. tatsch Glatze; etwas Breitgefallenes, -gedrucktes; sitzen gebliebenes Backwerk. Cf. 74B18.

3B1. Swiss blösch Kuh mit Blässe; Kahlkopf, Swab. blass weisser Fleck auf der Stirn; Kahlkopf, Fris. bles idem, DuDial. blesse idem, Du., Fris. blier idem. Cf. 4A1; Arnl. 9:21.

3B2. Sax. vollmond Glatze.

3B3. GerDial. blutti Glatze. Cf. 1F1.

\section{Forehead}

The forehead is described (A) as that which gleams, shines; (B) as something flat; (C) as a dome or peak; (D) as the brain.

4A1. Swiss blässe Stirnfläche; Pferd mit Blässlein, Thur., Lothr. bläss idem, Als. bless idem, Swab. blasse idem, NE. blaze. Cf. 3B1.

4A2. Flem. star, ster forehead, MLG. sterne Stern, sternartiger Fleck, Du. ster, star star; white spot on a horse's forehead, NE. star idem, OHG. sterro Stern.

4A3. Hess. schnippe forehead; upper nose of animals, LG. schnipp geflecktes Tier; Pferd mit Blässe; Taube mit Flecken.

4B1. Westf. platte Stirn; Scheitel; Kopf.

4C1. Swiss guppel Wölbung; gewölbte Stirn; Buckel, Ital. cupola dome. Cf. 89A4.

4C2. Swab. gibel Giebel; Gipfel; Stirn.

4D1. Swab. hirn Hirn; Stirn. Cf. 2M1.

\section{HAIR}

The hair is described (A) as a tuft, bunch, bundle, knob, knot, and the like; (B) as a curl, twist, roll; (C) as something woolly; 
(D) as a tousle, tangle; (E) as something hanging, dangling, flapping; (F) as something cut off, shorn, or plucked out; (G) as a strip, something elongated; $(\mathrm{H})$ as something bristly, like stubble; $(\mathrm{K})$ as a point, spout, horn, peak, and the like; (L) as a covering; (M) as a fringe; (N) as spectacles (of hair about the eyes); (O) as something sleek; (P) as a cowlick; (Q) as a collection of things belonging together; $(\mathrm{R})$ as an evening flower.

5A1. Bav. bibel Haarschopf. Cf. 2B2.

5A2. Bav. borzen, Swab. burz Büschel Kopfhaar, Zopf. Cf. $90 \mathrm{~A} 19$.

5A3. Swab. busch Gebüsch; Haarbüschel am Fessel des Pferdes; struppiges Haar, NE. bush.

5A4. Swab. butsch etwas Zusammengebundenes; Federschopf; Haarschopf; aufgewickelter Zopf, Swiss putsch Busch; Büschel Haare, Federn; Gebund, Thur. idem; aufgetürmtes Haar der Mädchen, Haarwulst, Lothr. bitsch Zopf; Haarbüschel. Cf. 34B1.

5A6. Thur. kauz Eule; die am Hinterkopf kranzartig gewundenen Haarflechten, Wald. kauts zusammengeflochtene Zöpfe auf dem Kopf, Swiss chuz Kauz; borstiges, struppiges Haar; Abfall vom Spinnen; Werg an der Kunkel.

5A7. Swiss üwel, üel Eule; Person mit verworrenen Haaren; struppiges, ungekämmtes Haar; aufgelöstes Haar der Frauen, Sax. bu-eule unordenliches Kopfhaar.

5A8. EDial. bump woman's hair tied into a knot behind. Cf. 90A17.

5A9. Als. buppe pine cone; Haarwulst, Swab. poppel Knauel Faden, Wolle; kugelförmige Früchte, Swiss puppe Auswuchs an Holz, am Brotlaib. Cf. 81B8.

5A10. Swiss buz kleines, unansehnliches Ding; Knötchen; dichter, voller, etwas ungeordneter Haarschopf, NHG. butzen Klumpen; Schnuppe am Licht; Kerngehäuse im Obst, MHG. butze Klumpen, LG. butt stumpf, kurz und dick. Cf. 81B5.

5A11. Swiss pfuz wirrer Haarbusch, Swab. pfauzig zerrauft, struppig vom Haar. F. connects with pfausig aufgeblasen, aufgedunsen.

5A12. Swiss fotze Quaste; Flöckchen von Fädchen; Baumwolle; Zotte; Haarlocke, Swab. Abwerg von Hanf, Flachs. Cf. 92C4, 127A15, 74B4; Arnl. 143:43. 
5A13. NE. fluke lock of hair; waste cotton, lw. from Norw. floke a tangle, matted mass, ON. flókna become matted.

5A14. EDial. fuke lock of hair, $p l$. hair. From the preceding?

5A15. Sax. wisch Wisch; Haarschopf, NHG. gebundener Strohbüschel.

5A16. EDial. wizzie thick covering, wrapper; shock of hair, wizers tops of vegetables.

5A17. Thur. dolle grosse Locke, hochgekämmte Haarfrisur, dollen kräuseln, Obersächs. dolle grosse, wulstige Locke, Swab. Quaste, OHG. tola Weintraubenkamm. Cf. $2 \mathrm{C} 2$.

5A18. Neumärk. tole Locke, Haarbüschel, Fris. tol kleine Quaste, Haarlocke, MLG. tul Büschel Haare, Flocke, Fris. tulln kräuseln.

5A19. Lothr. dotsch, dutsch Kuchen; Haarwulst; aufgebundene Haare der Frauen, Als. dotsch verdorbene Teigmasse, dotschen Kuhkot; missratenes formlos zusammengedrucktes Backwerk.

5A20. Lothr. kadutsch Schopf, blend of ka(rtause) Genick, Schopf +dutsch. Cf. 5A19, 40A10.

5A21. LG. dunck abgeschorene Wolle, dunk Wergwickel, docke Puppe (Kuhn Z., IX, 393).

5A22. EDial. toor, tour var. form of tower small heap; knot of hair.

5A23. NE. topknot tuft of hair on the top of the head; knob of hair on the crown of the head. Cf. 2A9a.

5A24. Swiss schubel derbes Stück; Mundvoll; Erdscholle; Haarbüschel; Haufen, MHG., NHG. schober hoch und spitzig zulaufend aufgeschichteter Haufen; Bündel; Büschel, Sax. schiebel kleinere Anzahl, Menge; Büschel Haare, Bav. schubel Haufen; Büschel Haare. Cf. $2 \mathrm{C} 6$.

5A25. Prus. schubs, schups Schopf. Grimm (IX, 2029): zu schopf, schups, schubs Stoss.

5A26. NHG. schock 60 Stück; Haufen von Garben; Schopf, NE. shock group of sheaves; thick, disordered mass of hair.

5A27. Sax. sturzel hervorstehender Stumpf; verstümmelter Finger; $M z$. kurz abgeschnittene und in die Höhe gerichtete Haare, NHG. sturzel stumpfes Ende; Baumstumpf, MHG. sturzel idem. 
5A28. Swiss gogg hohe Frisur, in die Höhe gestrichenes Haupthaar, goggel grosse Haube. Perhaps from Fr. coq as the hair thus combed was compared with a cock's comb (Schw.).

5A29. Swiss gügi Kranz der Haare hinten am Kopfe; aufgerollte Haarzöpfe. Cf. 81B12.

5A30. EDial. cob top, summit; tuft or bunch of hair on the forehead; lump, small round mass; nut, WFlem. kobbe tuft, crest; thick curly hair, Flem. kobbejager upstanding hair. Cf. 34B5, $126 \mathrm{~A} 12$.

5A31. Lothr. kowel Schopf; Knäuel, Als. kobel struppige Stelle des Hinterkopfes; langes Haar zw. den Hörnern der Rinder, Swiss kobel Haube; überhängender Fels. Cf. 34B6.

5A32. Sax. kolbe Kolben; kurzes Haar am Vorderkopf, NHG. kolben Stiel, Stab mit dickem Ende. Cf. 123C20, 68A4.

5A33. Prus. klatte verwirrtes Haar; angetrocknetes Klümpchen, klatter Klette; zusammenhangende Masse, Swiss chlette idem.

5A34. DuDial. klis burr; braid of hair, MDu. clisse burr, Du. klis burr, entangled knot (of hair).

5A35. Swiss chrübis Schopf. Cf. 2A21.

5A36. Sax. quaste Haarschopf, MHG. Büschel, Pinsel, Troddel.

5A37. EDial. hock shock of hair. Cf. 89A7.

5A38. NHG. holle Haarschopf, Swiss hulle grosses, ungeordnetes Haar der Weiber; woman's bustle. Cf. 2 G5.

5A39. Swiss huppe buschige Quaste; kugelförmiger, knollenförmiger Auswuchs; aufgerichteter Haarbüschel auf dem Kopf; das stumpfe Ende des Eies, hup convex, Swab. huppe Art Haube; kleines Hautgeschwür. Cf. 34B3.

5A40. EDial. rose tuft of bristles on a pig; division of the hair on horses, oxen.

5A41. Swiss ruggele Locke; kleines Ding, Tier, Als. grugele kleine Kugel; Knäuel Wolle; kleines dickes Kind.

5A42. Thur., Sax. nest Nest; aufgebundenes Haar, Wald. zusammengewundene Zöpfe, Swab. idem, webernest fehlerhaft gewobene Stelle, wo eine Masche fallen gelassen ist. Cf. 82A3, 85A37, 90A51, 96, 127A20.

5A43. Swiss nubel üppiger Haarwuchs, Schopf, LG. knubbe knot; knob, lump, NE. knub small lump; swelling, boil. 
5B1. NE. frizzle a curl; lock of hair crisped, verb curl, frizz.

5B2. Swab. driller drehende Bewegung; Haarwickel rechts und links vom Scheitel, drillen kreisend herumbewegen, MHG. gedrollen rund gedreht.

5B3. MLG. dwerl, dwarl Wirbel; Locke, OHG. thwiril Quirl. 5B3a. Als. troller Haargeflecht. Cf. 26E6.

5B4. Bav. schnecke Schnecke; geringelte Haarlocke. Cf. $123 \mathrm{E} 6,127 \mathrm{~L} 4$.

5B5. MDu. crulle, crolle, Du. krul Locke, MLG. krul Haarschopf, gekräuseltes Haar, MHG. krolle Locke, krūs kraus, NE. curl. Cf. 5B6, $123 \mathrm{Q} 1$.

5B6. Lothr. krausel Haarlocke, Prus. kruschel, Lothr. krusel, Als. krusele, Swiss chruselen idem, Als. kruse langes, gekräuseltes Haar, NHG. kraus. Cf. 5B5.

5B7. Lothr., Als. kruwel Locke, Kraushaar, Als. kruwelen kräuseln.

5B8. NE. crook bend; bent or curved part; lock of hair, verb bend; curl.

5B9. EDial. link lock of hair. Cf. 149C1.

5B10. Swiss ribel zugedrehter Wisch; kreisförmig gewundene Haare, Als. Strohwisch, Steir. rippel idem.

5B11. Swiss rigel Ringellocke, Bav. kleines Bund Stroh, etc., Strohwisch, ON. riga Krümmung, Biegung. Cf. 92D2.

5B12. Swiss rolle walzenförmig Zusammengerolltes; Haarlocke, Krulle, NE. roll.

5B13. Thur. wickel buschiges lockiges Haar, Als. wick unordentliches, verwickeltes Haar; dicke Haarsträhnen, Cologne weckel Schopf, NHG. wickel Flachs, Wolle um den Rockenstock; etwas Zusammengerolltes; Haarschopf, wickeln umwinden.

5C1. Als. belz Tierpelz; dickes Haar, Swiss Pelzähnliches; dichter Haarwuchs, Swab. pelz idem, OHG. pelliz, belliz Pelz. Cf. 127G1.

$5 \mathrm{C} 2$. Swiss bänzeni gekräuselte Haarlocken, benz Kosename des Schafes, eines plumpen dichtwolligen Schafes.

5C3. Swiss bausel etwas Wolliges, sich weich Anfühlendes; Troddel; Kosename der Katze; Haarbüschel am Schwanzende des Eichhörnchens, bausi Flocke Wolle, bausle Zotte, zottiges Ende des Haarzopfes. Cf. 127G5. 
5C4. Swiss budel Pudel-Hund; Kalb mit langen struppigen Haaren; Mensch mit langen krausen Haaren; Staubwischer mit langen Fäden; langes wolliges Haar, ungekämmtes Haar; crines genit. fem. Cf. 90A15.

5C5. EDial. flick fur of a hare or cat; fluff, dust collected into a light down, verb shoot so as to scatter the fur or feathers, var. of fix fur of various animals. Of unknown origin, possibly connected with $f l y$, verb (NED.), or NE. flock.

5C6. Swab. mull Katze; Pelz; wollige, federartige Blüten; Wollgras; weibl. Schamhaare, moll weibl. Schamhaare.

5C7. Swab. musel Katze; Pelz; Weidenblüte; das Weiche im Brot. Cf. 5 C6.

5C8. Oberh. wolle dickes, krauses Haar, wulle verworrene Haare, NHG. wolle sheep's wool; down, wool of plants.

5D1. EDial. jersey wool which has been combed out but not spun; yarn; contemptuous for a head of hair.

5D2. EDial. powse a curly head of hair, verb pull the hair, subst. peas, beans, pulse; fragments of pods after threshing pulse or clover when the seed has been milled out.

5D3. Sax. fitzer Haar, Obersax. fitz Wirrwarr von Fäden, OHG. fizza die Fadenenden des alten Aufzuges zum Anknüpfen des neuen, Norw. fit foot, the end of a woven piece. Compare Arnl. 84:01.

5D4. EDial. flax hair of an animal when detached from the skin; material resembling the fibers of the flax plant; a variety of asbestos.

5D5. EDial. tat tuft of hair, wool; matted mass, tatty tangled, matted, unkempt, tat to mat, entangle.

5D6. EDial. tangle the long fiber of a root; lock of hair; anything hanging, as a torn piece of a dress.

5D7. EDial. tussock tuft of coarse grass; tuft of hair, tooskie tuft of hair, NIcel. tuska rag, tatter, MHG, züsach Gestrüpp; Haarlocke, zerzūsen zerzausen, NE. touse pull about roughly.

5D8. EDial. tow flax, hemp for spinning; hair, tow out unravel.

5D9. Wald. tralj Gitter; verwirte Haarlocke, Lothr. tralljen Eisenstäbe vor den Fenstern; Gitterwerk.

5D10. Kärnt. tschouder, tschuder, tschoderle zerzaustes, buschichtes Haar, tschoudern bei den Haaren reissen; schütteln; 
schaudern. This word probably has LG. $d$ as does schaudern. Cf. 5D11, 12.

5D11. Swiss schudel wirres, zerzaustes, ungekämmtes Haupt, haar; starker Haarwuchs, schuderen die Federn emporrichten, schuderig zerzaust, struppig.

5D12. Kärnt. tschutte dichtes, zerzaustes Haar, tschuttern beben, schlottern, NHG. schütteln shake, schütten haufend ausgiessenschutt Geschüttetes. Cf. 5D10, $2 \mathrm{~A} 12$.

5D13. NHG. strobel Schopf mit starren, wirren Haaren, Lothr. struwel idem, struwatz Zottel, Haarbüschel, Als. strubel zerzaustes Haar. Cf. 32A3.

5D14. Swiss genisch dichtes in einander verschlungenes struppiges, verworrenes Haar, nische Schopf, verworrenes Haupthaar, nischen kämmen, genischig filzig, dicht verwachsen, vom Gras.

5D15. Kärnt. glotte ungekämmtes Haar, eine einzelne zerzauste Locke, glottat mit zerzausten Haaren, Swiss gelottert locker, lose, schlaff.

5D16. Swab. hanf Hanf; Haar.

5D17. EDial. harigalds, haricles, etc., viscera of an animal; locks of hair, probably from Fr. haricot a dish of boiled livers, hash (Jam.).

5D18. Swiss hurreli wirres Haupthaar, hurri einer mit wirrem Haar, hurrschen verwirren, hurrli wer sich überschnell bewegt, MHG. hurren sich schnell bewegen, NorwDial. hurra whir, whirl, NE. hurry.

5D19. EDial. raploch coarse woolen cloth; skin of a hare littered in March and killed at the end of the year, adj. homespun, rough.

5D20. EDial. roosk luxuriant growth of hair, rooskie unkempt, towsy, roosk to frizzle up.

5D21. Lothr. meissel Zopf geflochtener Haare, NHG. Scharpie, MHG. meizel Abgerupftes, Scharpie.

$5 \mathrm{E} 1$. Swiss umhang was an etwas umhängt wird; in Zöpfchen geflochtenes Haar, das an den Schläfen herabhängt.

5E2. Wald. bise kleiner, ungefügiger Zopf, bisen rennen, OHG. bisen in Brunst hin- und herrennen.

5E3. Swiss bluetsüger Blutegel; lange, freihangende, schlanke Locke, lit. bloodsucker.

5E4. EDial. wattle lock of hair. Cf. 14A5. 
5E5. EDial. daberlick kind of long seaweed; hair of the head when hanging in lank, tangled, and separate locks; any wet, dirty strap of cloth or leather.

5E6. Sax. zolk Büschel verwirrter Haare, zulkerch etwas Herabhängendes, Bav. zulcher herabhängender Fetzen, Als. zolge Ablaufröhre an einem grossen Fasstrichter; herunterhängender Rotz. Cf. 9A18.

5E7. Swiss halftere Halfter; Strick; Haarflechte, NHG. halfter.

5E8. Swiss hatsch ungekämmtes Haupthaar; ein hinter den andern gebliebener Schnitter; wer sich langsam und schwerfällig fortbewegt, Swab. hätsch schleppender Gang; Mensch der schleppend geht, Swiss hätschen schleppend gehen; sich nachlässig kleiden.

5E9. MLG. hovet-loppe Haupthaar, hovet Haupt, Swab. lopper locker, schlaff, Als. lopperig locker, klappernd.

5E10. Lothr. kartusen Schopf, Als. kartus Karthäuserkloster, Kapuze; Schopf. Cf. 40F2.

5E11. Swab. kengel Schwengel, Kloppel der Glocke; herabhängender Nasenschleim; Abteilung Haare, wie man sie beim Flechten macht, kengelen baumeln.

5E12. Swiss, Thur., Swab., Sax. nüschel Schopf. Cf. 2K1.

5E13. NE. list division of a head of hair, of the beard. Cf. $25 \mathrm{D} 13$.

5F1. Bav. schier, scher, schir Haare am Kamm des Pferdes, Mähne; Haarbüschel am Kopf des Stiers, Lothr. schur lange Reihe gemähten Grases, Bav. schur das Abmähen, Scheren; was abgemäht wird.

5F2. Oberh. strupf Büschel Haare, NHG. strupfen, strüpfen aufstreifen, abstreifen, NE. strip.

5F3. DuDial. roof the wool shorn annually from a sheep, Du. robbery, NHG. raub booty, OE. rēofan rend, rive.

5G1. Bav. schlick Zopf Haares; Büschel Flachses, NHG. ein der Länge nach ausgezogenes Flachsbündel; langer Haarzopf; Schlinge, Masche, Strick, Steir. Schafwollabfall, beim Kämmen.

5G2. EDial. strumel loose, long head of hair, MLG. stremel langer, schmaler Streifen.

5G3. EDial. swathe a baby's binder; wreath of mist; lock of hair, NE. bandage, OE. swapu bandage, fillet. 
5H1. Swiss, Swab., etc. bürste wie nhd.; struppiges Kopfhaar, OHG. borst, burst ein starres Haar. Cf. Arnl. 162:11.

$5 \mathrm{H} 2$. Als. widerburst langes, struppiges Haar; Haarwirbel, Lothr. widderbuscht gesträubter Haarbüschel, in die Höhe gekämmtes Haar über der Stirne. Cf. $5 \mathrm{H} 1$.

5H3. EDial. pen feather, quill; $p l$. long hairs of a deer; spigot, Lat. penna feather, pen. Cf. $174 \mathrm{C} 1,127 \mathrm{~S} 1$.

5H4. Swab. (Rotwelsch) hälmle Haare, NHG. halm.

5H5. Als. kambes Schopf, Swiss chamb Haarkamm; Mähne; Scheitel des Menschen; Kamm der Hühner; Bartlappen derselben; Halsrücken des Pferdes; Bergrücken.

5H6. Lothr. stopple Stoppeln; kurzes Haar beim Menschen; Flaum bei Vögeln.

5K1. Swab. bockhörner vier Haarzöpfe einer Frau, Swiss horn Haarbüschel, der auf dem Kopf hervorragt. Cf. 53C1.

5K2. DuDial. pijl a thin shoot; a hair, Du. dart; shaft of an arrow; stalk, OE. pīl something pointed, thorn.

5K3. Du. tuit point, sharp end; pointed braid of hair; spigot.

5K4. EDial. widower's peak point of hair growing in the middle of a man's forehead.

5L1. NE. coat outer garment; natural covering of animals, as hair, wool, etc.

5L2. EDial. thatch thatch; head of hair, OE. poec thatch.

5L3. Sax. schaubdach Strohdach; das verschnittene Haupthaar, Bav. schaupen Schopf; dichter Busch von Haaren, etc., NHG. schaub, MHG. schoub Strohbund, -büschel+dach Dach, NE. thatch.

5M1. Sax. simpelfranse in die Stirn hängendes Haar, simpel Dummkopf + franse fringe.

5M2. NE. fringe portion of the hair brushed forward and cut off.

5M3. EDial. skirt short wool on the forehead, belly and legs of sheep, NE. border, rim, outer portion. Cf. 112D1.

5 N1. Swiss brille Brille; grauer Haarkranz um die Augen (of old horses).

501. Oberh. schnatz das glatt gekämmte und zierl. gewundene Haar der Mädchen. Grimm: schnatz Schnitz; das geflochtene und um die Nadel gewickelte Haar der Frauen, schnatzen schnitzen; prächtig putzen, Kurhess. schnetzer reinlich, ordentlich. 
502. EDial. slick down of rabbits, adj. sleek, smooth; polished, shining, NE. sleek.

5P1. NE. cowlick a tuft of hair which presents the appearance of hair that has been licked by a cow out of its proper position, also called calflick.

5Q1. EDial. laughter sitting of eggs; brood of chickens; litter of pigs; layer, stratum; lock of hair or wool, ON. lātr Lagerstätte.

5R1. Swiss chilt-blueme Abendlichtnelke; graue Haare, chilt Abendtätigkeit, OHG. chilti-werch Abendwerk.

\section{FACE}

The face is described (A) as the appearance, looks, manner; (B) as something which exhibits the feelings or character, as a weeper, cloudy or gloomy thing, a pouter, etc.; (C) as that which eats, "slorps," snuffles, hunts about and chooses out; (D) as that which bellows, mumbles, gabbles; (E) as a rounding object, swelling, something puffed up, soft mass, etc.; (F) as something contracted, shriveled; (G) as something protruding, projecting, such as a point, spout, etc.; (H) as a flap; (K) as something gaping; (L) as something gleaming; (M) as that which grins; $(\mathrm{N})$ as something ridiculous; (O) as a mask; (P) as a wig; (Q) as a grating (crossbars); (R) as a songbook; (S) as a battery; (T) as a bakery; (U) unclassified.

6A1. MDu. aenschijn that which is seen of a person; the face as mirror of character and as part of the body, schinen shine, be visible, MLG. anschin Angesicht; Gegenwart, Du. aanschijn face, MHG. anschīn Erscheinung; Deutlichkeit.

6A2. Du. gelaat manner, bearing; face; facial expression, EFris. gelät face, MDu. ghelaet manner, bearing, MHG. gelāzen Gebahren.

6B1. Swiss boffele unfreundliches, hässliches Gesicht, MDu. boffe, buffe thick face; clap, smack, Swiss boffen vom Rindvieh, finster heraufschauen und durch die Nase blasen; schmollen; schnarchen, NHG. büffen bauschen machen.

6B2. Swiss briegg zum Weinen verzogenes Gesicht, briegge verzerrtes Gesicht, brieggen den Mund verziehen, Swab. briegen weinen, MHG. brieke Flenngesicht, OHG. prieken machondo ora torquendo. 
6B3. Als. flenne Gesicht, Sax. flunsch verzogener Mund, Schles., Thur. verzogenes Gesicht, OHG. flannen den Mund verziehen. Compare Arnl. 26:17.

6B4. Swiss gränne Fratze; verzerrtes, hässlich weinerliches Gesicht, Swab. idem, MHG. grannen weinen, flennen.

6B5. Swiss chumber Not, Bedrängniss; bekümmertes Gesicht MHG. kummer Unrat; Belastung, Bedrängnis, Not.

6B6. Als. lentsche verzogenes Gesicht, Swab. luntsch idem. Cf. $7 \mathrm{~K} 9$.

6B7. Swiss nibel umwölkte Miene, finsteres Gesicht; Hängemaul, genibel Nebelwetter, Swab. nibel weinerl. Gesicht, genibeltes angesicht nebulosa facies, NHG. nebel.

6C1. DuDial. azent face, MDu. āsen eat, feed, Du. azen prey; feed upon.

6C2. Kärnt. fresse Gesicht, gefriss, Bav. gefriss, Nos. gefrīs, Swab., Steir. fresse Habkern, Swab. gefräss Gesicht. Cf. 7A3.

6C3. Sax. laffe, Swiss läff Gesicht. Cf. 7C11.

6C4. Als. lafete Lafette; Gesicht, Sax. lafette idem. Blend of Sax. la $(f f e)$ face+(la)ffette gun carriage. Cf. the preceding and 90V1, 25D10, 7C12.

6C5. DuDial. snoefel face, snoefelen smell, rummage. Cf. 9L16.

6C6. Als. schneik abgemagertes, blasses Gesicht. Cf. 7C6.

6D1. Swiss brieschele saures Gesicht, gebriesch Gebrüll, brieschen brüllen. Cf. 7D12.

6D2. Sax. plappe Gesicht. Cf. 7D6.

6D2a. Sax. plarpe trotziges Gesicht. Cf. 7D8.

6D3. Swiss müffi saures Gesicht. Cf. 7D27.

6D4. Swiss mügge saures Gesicht; mürrisches Weib; Murmeltier, mauggere, mäuggel saures, finsteres Gesicht, müggen muhen, brüllen; stöhnen; vor sich hin brummen. Cf. 7D28.

6E1. Als. bibele feistes Gesicht. Cf. 2B2.

6E2. Swiss bläster mürrisches Gesicht. Cf. 40A3.

6E3. Swiss bröggel verdriessliches Gesicht, brögge Grimasse, Swab. broglen prahlen, gross tun, MHG. brogen sich erheben; gross tun.

6E4. Sax. parabel Gesicht. Cf. $2 \mathrm{~A} 7$.

6E5. Kärnt. pappn Gesicht. Cf. 7A2. 
6E6. Tirol. pfouse finsteres Gesicht, Swab. pfauskopf beleidigtes Gesicht, pfaus dick, aufgedunsen, early NHG. pawsen strotzen.

6E7. DuDial. dotterd face. Cf. 114A1.

6E7a. Thur. metsche böses weinerliches Gesicht. Cf. 7H17.

6E8. Flem. moel face; mouth, ON. müli muzzle; mountain peak; abgerundeter Felsen.

6E9. Swiss mol Weichtier; unförmlich Grosses; Mensch mit Pausbacken; pausbackiges Gesicht; dicker Kopf, Swab. molle dicker, aufgedunsener Mensch; Schmerbauch, NHG. moll überreif (Obst), OHG. molawēn melt.

6E10. EDial. mug face. Cf. $7 \mathrm{H} 19$.

6E11. Swiss müwel verdriessliches Gesicht. Cf. 7H16.

6F1. Swiss nīffi verzogenes Gesicht, Grimasse, nīffen das Gesicht verzerren, verziehen, verniffen zusammengeschrumpft, verkümmert; nicht ausgewachsen; unansehnlich, nifeli etwas Winziges, Verkümmertes.

6G1. Du. toot face; kind of cap; kind of horn, MDu. tote apex. Cf. $7 \mathrm{~J} 5$.

6G2. Swab. gosche Gesicht, Lothr. gosch idem. Cf. 7J2.

6G3. Steir. grantze Gesicht; Schweinsrüssel, Swiss granse Schiffsschnabel; Schweinsrüssel; schnabelförmige vorspringende Bergeshöhe, gränse Maul, MHG. grans Schnabel; Rüssel; Maul; Schiffsschnabel. Compare Arnl. 23:18.

6H1. Steir. lesche Gesicht. Cf. 8C10.

6H2. Swiss litzi Gesicht. Cf. 7K7.

6K1. Swab. blärre, blarre vom Gaffen verzerrtes Gesicht; grosse Fläche Ackerfeldes; das in die Augen fallen, blarren alles sehen wollen, gaffen, Bav. blerrer offener, freier Platz, MHG. plarren gaffen.

6K2. Als. flarr saures Gesicht. Cf. 7K2.

6K3. Swiss gläff verzogenes Gesicht; heiterer Himmel, gläffen gaffen.

6L1. Als. mond Mond; rotglänzendes, feistes Gesicht.

6L2. Swiss blassi Gesicht mit Flecken; schmutziges Gesicht. Cf. $4 \mathrm{~A} 1$.

6M1. Als. zänn verzogenes, grimmiges Gesicht, zännen zornig oder zum Lachen das Gesicht verziehen, OHG. zannen idem. 
6N1. NHG. fratze ins Lächerliche verzogenes entstelltes Gesicht, Ital. frasche Possen, Albernheiten.

601. Sax., Swab. larve Gesicht, NHG. mask. Cf. 701.

602. EDial. mask face, without idea of disguise; hunting term for a fox's head.

6P1. EDial. giz, gizzy, jeezy wig; face, NE. gizz wig, origin unknown (NED.).

6Q1. Swiss gufi Wagenkasten aus Brettern, Leitern oder Flechtwerk; Sehrotleiter aus 2 durch Querleisten verbundenen Balken zum Transport von Fässern; Kasten am Milchkarren in welchen die Milchtansen gestellt werden; Holzgestell zum Tragen auf dem Rücken; verzogenes Gesicht.

6R1. Sax. gesangbuch Gesicht.

6S1. Sax. batterie Gesicht.

6T1. Flem. bakkes face. Cf. 7M1.

6U1. Sax. täte Gesicht; Nase, Fr. tête?

6U2. Lux. fons Gesicht.

\section{Mouth}

The mouth is described (A) as that which eats, tastes, gobbles up; (B) as that which breaks, crushes, bites, gnaws, nibbles; (C) as that which "slorps," slobbers, snuffles, licks, acts in a sloppy fashion; (D) as that which talks, prattles, gabbles, barks, bellows, mumbles, snores, buzzes, rasps, blows, and the like; (E) as that which snaps, snatches; (F) as a receptacle, such as a basket, drawer, sack, hole, crevice, trap; $(\mathrm{G})$ as that which gapes; $(\mathrm{H})$ as a swelling, something full, soft mass, lump, chunk, and the like; $(J)$ as something projecting, protruding, such as a point, spout, snout, and the like; (K) as something loose, lolling, hanging, a flap; (L) as a brim, border; (M) as a bakery; (N) as a shop; (O) as a mask; (P) as a privy; (Q) unclassified.

7A1. Westf. brōdeske Mund. From brod+es(sen)+dimin. ending.

7A2. Steir. pape, papn, Thur., Kärnt. pappe, Egerl. pappm Mund, Thur. pappen essen, NHG. pappe Mehlbrei, MDu. Brei. Cf. 6 E5. 
7A3. NHG. fresse, Dial. gefräss, LG., Fris. fret mouth. Cf. $6 \mathrm{C} 2,9 \mathrm{~N} 1$.

7A4. EDial. gobbler mouth, gobble eat greedily.

7A5. Swiss lafuttere mouth. From la(fete)+futteren. Cf. 6 C4.

7A6. Sax. schmecker Mund, NHG. schmecken.

7A7. Thur. schlunte Maul, OHG. slintan verschlingen. Compare Arnl. 43:31.

7B1. Steir. brechel wie Hd.; Mund, brechen break.

7B2. Westf. mappe Mund, Thur. mappeln kauen, EDial. map nibble.

7B3. Kärnt., Tirol. mulle Mund, Cologne mul idem, Swiss mullen kauen, mit übervollem Munde und mit sichtbaren Kaubewegungen, OHG. mullen zermalmen.

7B4. Bav. rützen Mund, Swiss verb nagen.

7C1. EDial. flouch awkward mouth. From $f($ orch $)$ to spread the mouth from affectation $+(p)$ ouch to swallow, eat greedily.

7C1a. Swiss gätsche Maul, gätschen mit klatschendem schmatzendem Tone essen; platschern, gatscher Fresser.

7C2. Lux. sabbel Geifer; Maul, sabbelen geifern.

7C3. Nos. schlabber, Sax. schlabberloch mouth, Wald. schläw, Flem. slabbaris idem, NHG. schlabbern wie ein Hund schlürfend essen; schwatzen, plappern. Cf. 22E6, $104 \mathrm{G} 1$.

7C4. Thur. schlatte, Hess. schlätte, Thur. schlitte, schlutte mouth, Sax. schlattern schluckweise trinken, EDial. slatter spill, splash, slop; upset; waste.

7C5. Bav. schler Mund, schliren naschen, lecken, Oberh. schlerr Mund, LG. sliren schlürfen, schlecken.

7C6. Als. schneik Maul, Mund; Person die im Essen wählerisch ist, schneiken neugierig suchen; wählerisch sein, beschneiken beschnüffeln, MHG. snöuken heimlich gehen, schnobern. Cf. 6C6.

7C7. Als. schnuffel Mund. Cf. 9L16.

7C7a. DuDial. snoevel mouth, MDu. snoeven heftig atmen, schneutzen. Cf. 6 C5.

7C8. Cologne schnüss, Lothr. schniss, Lux. schnöss Maul. Cf. 9L21.

7C9. Swiss, Thur., Sax. nüschel Maul, nieschen weit aufgesperrtes Maul. Cf. 2K1. 
7C10. Kärnt., Tirol. labratsche Mund. From la(bern) leckend trinken+bratsche mouth. Cf. $7 \mathrm{H} 3$.

7C10a. Bav. trenschen Mund, Swab. trenzen tropfenweise fallen lassen; langsam machen, trenzer wer trenzt; herabfallender Tropfen.

7C11. Als. läff Mund; Mensch der beim Gehen wackelt, Swiss läff, Sax. lawwe, labbe, Thur. lewe, labbe mouth. Cf. 8 F2 and following.

7C12. Swiss, Als. lafete, Sax. laffeet, laffette, Swab. lafette Mund (derb). Cf. 6C4.

7C13. Bav. lefern Hängemaul, Swab. leffe Maul, laffen lecken, schlürfen, Schles. loppe Mund, Maul, NE. to lap. Cf. 7C11.

7C14. Egerl. lapprn Mund, Swab. lappe, lappel mouth, lappen schlürfend auflecken, läpperig dünnflüssig, lapper Kuhfladen.

7C15. Swab. läutsch wüster, hässlicher Mund, lutschen saugen, schlotzen, Swiss lutsch Saugbeutel.

7D1. DuDial. babbel mouth; gills; babbling; chewing, babbelen chew, tattle, gabble, Cologne bäbbel, Thur. bäppe, babrich Mund, bappeln prattle, NE. babble.

7D2. Kärnt. paffe, Aach. bäff Maul, paffeln, Swiss bafflen chatter, prattle, gabble.

7D3. Fris. blaeijer mouth, babbler, blaeije to pant, lick; cry, OHG. blāen blasen. Cf. 22E1.

7D4. Kärnt. plase Mund, NHG. blasen.

7D5. Cologne blaffet mouth, NHG., MHG. blaffen bellen.

7D6. Sax. plappe, pleppe, Prus. plapper, Steir. plepper Mund, NHG. plappern. Cf. 6D2.

7D7. EFris. pläter grobes Maul, plätern plappern, plaudern, MLG. pleteren schwatzen, plaudern.

7D8. Sax. plarpe Mund. Blend of plar(re) (7D9) and (plap)pe (7D6).

7D9. Prus. plarr, plärr, plärre Maul, Sax. plärre Mund, plärren weinen, schlecht singen; lärmen, Kärnt. plerre Maul, Swiss plarren muhen, brüllen.

7D10. Sax. plauze Mund, plauzen krachen; geräuschvoll, schwer auffallen, plauz heftiger Knall.

7D11. Swiss bräll breiter Mund mit dicken Lippen; wüstes Maul, brällen brüllen; laut weinen; prahlen, NHG. brüllen.

7D12. Swiss brietscheli verzogener Mund, brietschen weinen. Cf. $6 \mathrm{D} 1$. 
7D12a. Kärnt. pratsche Maul; wer viel redet, Swab. bratschen plaudern.

7D13. Wald. proüt verzerrter Mund, Du. preutelen grumble, mumble.

7D13a. EFris. fäter grobes Maul, flätern schwatzen, plaudern, LG. fläter Plaudermaul, Schwätzer.

7D13b. Kärnt. fratsche, Bav. frotschen Mund, Kärnt. fratschn plaudern; wiederholt fragen, Bav. frötscheln idem.

7D14. Lux. gabber Mundwerk, NE. gab mouth; idle talk, gabble, MDu. gabben prattle, jabber, Lothr. gabber Mundwerk; Kinn, Prus. jabbel Mund, Schnauze, jabbeln viel und unnutz reden.

7D15. Prus. gibbel Mund, gibbeln schwatzen, plappern.

7D16. Swab. gir Mund, girren keifend schreien, gurren girren, knarren; zanken.

7D17. Tirol. güre Mund, gurren einen gurrenden, knurrenden Ton von sich geben. Cf. 7D16.

7D18. Fris. haffel mouth, haffelje gnaw; mumble, EFris. haffeln rasch und gierig essen und schlucken, WFris. haffelje kauen wie alte zahnlose Leute; viel und unaufhörlich schwatzen.

7D19. Prus. kautsche mouth, kautschen viel und anhaltend bellen; zanken, Swiss chautschen nagen, lange kauen; schwatzen; kläffen. Cf. 9L2.

7D20. Prus. keite mouth, keitern viel reden, schwatzen, keiter Schwätzer; kläffender Hund.

7D21. Prus. kōse Mund, kōsen reden, plaudern, plappern, OHG. chōse, MHG. kose Rede, Gespräch.

7D22. EFris. käkel Maul; Zank, Prus. Mund; Schwätzer, LG. kakel Plaudermaul, EFris. kakeln gackern, schreien. Cf. 9L4.

7D23. Thur., Sax. klappe, Swiss chlappe, DuDial. klep mouth, Swiss chlappen schlagen, prügeln; klingen; schwatzen, chlappere klapperndes Spielzeug; Plappermaul, Sax. zündnadelklappe grosser Mund. Cf. 22E3.

7D24. Flem. knebbel, knibbel mouth, knebbelen, knibbelen haggle, quarrel. Cf. 22E5.

7D25. Fris. knappert mouth, knap snap, clap, knappe to crack, snap.

7D26. Du. kweb mouth that is never still; person always talking, kwebbelen babble, chatter. Cf. $7 \mathrm{H} 15$. 
7D27. Swiss moffle, Swab. muffel Maul, mufflen murren, mäffelein kleiner Mund, Swiss mofflen langsam mit Mühe, Behagen kauen; undeutlich sprechen, Als. mäf Maul, Swiss möff Maul, möffi kleiner spitzer Mund, muffen schmollen, muffle grosser, vorstehender Mund, müffi verzogener Mund. Cf. 6D3, $13 \mathrm{~B} 2$.

7D28. Swiss müggel verzogener Mund. Cf. $6 \mathrm{D} 4$.

7D29. Steir. mummel mouth, Swiss idem; Mensch, der unverständlich vor sich spricht; Insekt, das beim Fliegen einen surrenden Ton hören lässt, mummelen murmeln, murren. Cf. 17A5.

7D30. Swiss mumpfel Mundvoll; unwillig verzogener Mund, Swab. das Weiche im Brot; Maul, grosser Mund, NE. mump mumble, mutter, Du. mompelen mumble.

7D31. Kärnt. mearn Mund, māren etwas weitlaufig erzählen, Swab. mären sagen, offenbaren.

7D32. DuDial. mörf $(t)$, murf mouth; grumbler; head, Du. murf mouth, beak, Bav. murfel kauendes Weib; Gesicht, Maul, Schnauze, Tirol. murfe mouth, NHG. murfeln mit geschlossenen Lippen kauen, MHG. murfen nagen.

7D33. Kärnt., Swiss murre mouth, Swab. verdriessliches Gesicht, murren brummen, knurren, Swiss murrlen dumpf tönen.

7D34. Als. raffele Klapper, Ratsche; geschwatziger Mund, Rap. rafl Raffel; Mund, zahnraffel Mund, Swiss rafflen Reibeisen; Gebiss; unermüdliches Maul, rafflen am Reibeisen zerreiben; an Holz nagen; klappern.

7D35. Prus. schabber mouth, schabbern viel schwatzen, reden, plappern, geschabber Geschwatz.

7D35a. Bav. schnauppen Mund. Cf. 9L17.

7D35b. Swiss sage, Prus. segg mouth, seggen sagen, NHG. sagen.

7D36. Fris. snater, snetter mouth, EFris. snater Plappermaul, Schwatzmaul, Du. mouth, snateren babble, gabble, Westf. sneter Mund, Prus. schnatter idem, NHG. schnatter. Cf. 9L12.

7D37. DuDial. snassel mouth, snasselen gabble, babble.

7D38. NHG. schnurre Schnarre, Knarre; Maul und Nase, schnurren hart und zitternd dumpfig tönen. Cf. 9L20, 35F2.

7D39. Prus. schnorgel, schnörgel, schnurgel Mund, schnorgeln durch die Nase laut atmen; schnarchen. Cf. 9L15.

7D40. Sax. schmatzmachine Mund, schmatz Kuss, Swiss schmatzen geräuschvoll essen, NE. smatter defile; prate; chatter. 
7D41. Sax. schwadder, schwade Mundwerk, schwadern viel schwatzen, suade Redegabe, MHG. swateren rauschen, klappern. Cf. 7D42, 43.

7D42. Sax. schwudel Mundwerk. Cf. 7D41.

7D43. Sax. schwarte Mundwerk. From schwade (7D41) (Wg.).

7D44. DuDial. tater mouth, EFris. tater, totter Plappermaul, Schwatzmaul, tatern, tottern plappern, schnattern, Du. tateren stammer, babble.

7D45. Als., Bav., Du. waffel mouth, Als. wafflen schwatzen, plaudern, gewaffels Klatscherei.

7D46. MLG. wasche Mund, Plappermaul, Bav. waschen Mund; Ohrfeige, MLG. waschen waschen; schwatzen, Als. wäschen idem.

7E1. Fris. happert, DuDial. hapsnap mouth, Du. happen, Fris. happe snap, snatch.

7E2. Fris. snappert mouth, snappe fassen, greifen, NHG. schnappen.

7E3. Bav. düschel Mund, Kuss, Swab. tusch schnelle Handbewegung, rascher Griff nach etwas, tuschen hastig nach etwas greifen.

7E4. Prus. husche Ohrfeige; Mund, huschen schnell, leicht, unbemerkt im Husch sich fortbewegen, NHG. husch grosse Eile.

7F1. Swab. brot-fotze Mund, fotze anus, cunnus.

7F2. Sax. futter-spalte Mund.

7F3. Schles. tasche Mund. Cf. 127B5.

7F4. Sax. kartoffelfalle Mund, falle trap.

7F5. EDial. trap trapdoor, hatch; mouth.

7F6. Prus. scheide mouth, NHG. längliche hohle Aussenbekleidung der Waffenklinge; dem Ähnliches. Cf. 127B6.

7F7. Cologne schören-döör Scheunen-tür; grosser Mund, schör Scheune, döör Tür.

7F8. Lothr. schublad, Sax. brotladen, Kärnt. tischlade, Swab. lade mouth.

7F9. Kärnt. gūfl Mund, Bav. gufel Höhlung in einer Felsenwand, Swiss gauf, Swab. gaufe, MHG. goufe hohle Hand. Compare Arnl. 58:01.

7F10. Kärnt. gēfl Mund, Bav. gaiffen auseinanderstehen. Cf. 7F9.

7F11. Westf. kaügatt Kauloch, Mund, gatt Loch. 
7F12. Swiss chämin Kamin; Mundöffnung, MHG. kamīn, kemin Feuerstätte, Ofen. Cf."10A3, 91A5.

7F13. Swiss chorb Korb; Hängemaul, Swab. brot-korb Mund. Cf. 83A3.

7F14. Swiss chlack Spalte, Riss; grosser Mund, suppen-chlack grosser Mund mit schmalen Lippen, Swab. klack Spalte, Ritze.

7F15. EFris. holster Holfter; grosses weites Maul, NE. holster. Cf. 85A25.

7F16. Swab., Swiss loch Mundöffnung, Sax. futter-luke, Kärnt. vaterunser-loch, Westf. -lok Mund.

7F17. EFris. rachen Rachen, aufgesperrtes weites Maul, bz. Maul,-Schlund, Kehle, Gurgel, NHG. Anfang der Halshöhle; aufgesperrtes Maul, MHG. rache Rachen, Gaumen. Compare Arnl. 43:41.

7G1. DuDial. gaapard person always gaping; mouth.

7G2. Thur. giffel, Swab. gaffel mouth, DuDial., Fris. gaffel wideopen mouth; two-tined fork, NHG. gaffen.

7G3. Kärnt., Swab., Steir. fletsche mouth, NHG. fletschen die zähne zeigen; ins Breite dehnen; flach, breit schlagen, MHG. vletzen ausbreiten, OHG. flaz flach. Cf. 61A2a.

7H1. EDial. buss clump, tussock; pouting mouth, NE. subst. and verb kiss, Swab. Hügel; Beule.

7H2. Swab. blätsche breiter, herabhängender Mund. Cf. 8D1.

$7 \mathrm{H} 3$. Thur. bratsche breitlippiges Maul, Kärnt. pratsche Maul. Cf. 50A1.

7H4. Kärnt. labratsche mouth. Cf. 7H3, 7C10.

7H5. Thur. britsch Pritsche; holzernes Lager, etc.; verzogener Mund, NHG. pritsche board seat, etc.; bat, mace. Cf. 50B12, 90I1, 92A2, 127D2.

7H6. Swab., Oberh. brutsche big protruding, wry mouth, Bav. brotschet breit gequetscht; aufgedunsen, dick.

7H7. Kärnt. pock Bock; Maul, NHG. bock buck; Balken, Klotz zum Stossen.

7H8. Swab. pfanne verzogener Mund, Swiss ein pfändlein machen den Mund zum Weinen verziehen. Cf. 90E8.

7H9. EDial. fuz-mun full mouth with protruding lips, fluz to blunt, turn up or jag at the edge; mun mouth. Compare Arnl. 26:01. 
7H10. Swiss flarz, flartsch breites, schwer wiegendes, schwer bewegl. Stück Erde; breit zum Weinen verzogener Mund; breite Masse oder Gestalt. Cf. 7K2.

7H11. Thur. dünschel schiefer Mund, Sax. dunsch idem; Kuss, dunsen aufgedunsen, MHG. sich dinsen anschwellen.

7H12. Swab. dutte verzogener Mund. Cf. 81B10.

7H13. Lux. schöpp, Bav., Neumark., Thur. schippe Schmollmund; Schaufel; Stülpe der Mütze, NHG. schüppe shovel.

7H14. EDial. gob, gub mouth, beak, gobbed full-lipped gob mass, lump; bunch.

7H15. Eichstatt. kwaaf Maul, Du. kwab quivering lobe, lump of flesh, kwabbig round and full; having lobes, NE. quave. Cf. 14A13.

7H16. Swiss mäuwi das Wiederkäuern; wiedergekäute Masse; Maul, müwel hässlich verzogener Mund, mauwen wiederkäuen, Bav. maue pulpa, das Fleisch an Früchten und Tieren, das nicht beinicht ist. Cf. 6E11, 113B9, 123C22, $138 \mathrm{~B} 1$.

7H17. Thur. metsche verzogener Mund, Swiss matsch breiartige, weiche Masse, Swab. idem; matsche dicke plumpe Frau. Cf. 6E7a.

7H18. DuDial. mörk head. Cf. 2 A35.

7H19. EDial. mug mouth; drinking vessel, jug, Norw. mugga cup, jug. Cf. 6E10, 90A49, 104B9.

7H20. Swiss mutti Hängemaul; Ziegenbock ohne Hörner; Huhn ohne Schwanz; Dickkopf; kurzer, dicker Gegenstand, mutt stumpf, grob und dick. Cf. 85A36, 127A29.

7J1. Swiss beffel, biffel Mund. Cf. 8A2.

7J2. NHG. gosche, gúsche Maul, Swiss gösche, götsch kurze Ausgussöffnung am Milchkruge; Mund und Kinn; verzogener Mund, Swab. gosche grosser Mund, Prus. gusch, Sax., Schles., Hess. gusche mouth, Swiss giesch grosses, weites Maul; grosses unförmliches Ding, Rap. kosche Maul, EDial. gush waterspout. Cf. 6G2, $8 \mathrm{~A} 5,127 \mathrm{~A} 22$.

7J3. Sax. galusche Mund. From gal(osche) Schuh (cf. Fr. menton de galoche protruding chin) $+($ g) usche (7J2).

7J4. Sax. karpfen-schnute "carp-snout"; mouth.

7J4a. Oberh. dutte Mund. Cf. 9F2.

7J5. DuDial. toot, teut spout; pointed end; mouth with protruding lips, mouth. Cf. 6G1. 
7J6. Tirol. zange Mund mit sichtbaren Zähnen, NHG. zange.

7J7. Prus. zinken Mund, NHG. tine, prong. Cf. 9B17.

7J8. Prus. schnibbe Schnabel, Mund; Nase; schnabelartig auslaufende Taillenspitze an Frauenkleidern, Du. sneb Schnabel. Cf. 123C13, Arnl. 23:40.

7K1. NHG. flabbe, flappe herabhängende Unterlippe; offenstehender Mund, MLG. vlabbe, Du. flab, fleb, Prus. flab, Westf. flabbe idem, Westf. flapps Mund, Lippe, NFris. flap Unterlippe, Sax., Thur. flappe hangende Schnauze oder Lippe, Mund, EDial. flep, fleb, flip lip, Icel. flipi untere Pferdelippe, NE. flabby. Compare Arnl. 26:18.

7K2. Swab. flärre breites Maul; dummes, grossmäuliges Gesicht, flärr Fetzen; herabhängender kleiner Hautteil; breites abgerissenes Stück Fleisch; breite, unförmliche, klaffende Wunde, Bav. flarre Lippe, Maul, Oberh. flerre etwas das klaffend voneinander steht; breites grosses Maul, von dem die Unterlippe herabhängt, MHG. vlarre, vlerre breite, unförmliche Wunde. Cf. 6K2, 7H10.

7K3. Eichstätt. slepern verzogener Mund, NHG. schleppen drag, trail.

7K4. Als. lattere Mund, Swiss lätter träger Mensch, strumpflätter Herunterrutschen der losen Strümpfe, lotteren wackeln, schlotterig hangen.

7K5. Als., Lothr., Bav. lätsch Mund, Swab. Masche, Schleife, grosses Endglied einer Kette; Mund mit herunterhängender Unterlippe, Lothr. lätschig locker, faul; läppisch, Swiss lätsch Hängemaul, Sax. litsche schiefer Mund, Flunsch. Cf. 8C9.

7K6. Steir. lesche Mund, Swiss lasche Hängemaul, Swab. läsch Maul. Cf. 7K5, 8C10.

7K7. Swiss litz Umgebogenes; Falte; Lappen am Kleid, Eselohr in einem Buch; Hängemaul. Cf. 7K5, 6H2.

7K8. 'Rap. lele, Swiss lälle Mund. Cf. 22A2.

7K9. Als. lentsche breites unförmliches Maul, Swab. luntsch Hängemaul, herumlenschen herumrutschen, lüntschen sich auf einem Stuhl nachlässig hin und her bewegen, Lothr. lintsch matt. Cf. 6B6, 26C1, $113 \mathrm{C} 4$.

7K10. LG. labbe Mund. Cf. 8C6. 
7L1. Thur., Swiss rand Rand; Mund, Westf. rant Lippe; Maul, Thur. ranft Brotende; Mund, NHG. rand Grenze einer Fläche; erhöhtes Ufer, MHG. rant Schildrand; Einfassung; Rand. Cf. 8E1.

7M1. Flem. bakkes mouth. A facetious compound of MDu. $b a c(k e)$ and huis under the influence of MDu. bac-huus bakery. Cf. 6T1. Franck: older forms are backhuys, bakkis, bakkus, from which came bakkes just as Westf. backes "kleines Haus, worin der Bauer seinen Backofen hat" came from MLG. backhüs bakery.

$7 \mathrm{~N} 1$. EDial. shop place where business is done; mouth.

701. Swab. larve mouth. Cf. 601.

7P1. Swab. laube Laube; Abort am Ende eines Ganges; Mund, Swiss idem. Similar comparisons occur in English.

7Q1. EDial. corp mouth, lips. Unexplained (Wr.).

\section{LIP}

The lip is described (A) as a swelling, something protruding, projecting; (B) as a hook, crook; (C) as something hanging, dangling, a flap; (D) as a blade, leaf; (E) as a border; (F) as that which mumbles, snorts, "slorps."

8A1. EDial. babber underlip, Du. babbe bib; swelling on the throat, NE. babber-lipped having thick projecting lips.

8A2. Bav. beffel, biffel zusammengedrückte vorstehende Lippen, Swiss befze lip, NHG. beffchen die zwei länglichen weissen herabhangenden Läppchen unter dem Kinne des Geistlichen, MLG. beffe Chorkappe, EFris. beffe, bef über die Fensterrahmen vorstehendes keilförmiges Gesims. Cf. 7J1, 13A2.

8A3. Oberh. brull dicke aufgeworfene Lippe, MG. brulle, prull dicker Mund, LG. prull boil, swelling, pral aus Fülle straff, NHG. prall. Cf. 100A3, 126A5.

8A3a. EDial. pough lower lip, NE. bag; swelling of the skin, verb hang loose, bag out, EFris. puche a boil (NED.).

8A4. EDial. gagger, gegger large ugly liquid or semi-liquid mass; underlip; large rugged cloud, verb cause to project, NE. gag to project.

8A5. Swab. gosche Lippe. Cf. 7J2.

8A6. Swab. gutsche-bock Kutschersitz, "Kutsche-bock"; hervorstehende Unterlippe. 
8A7. EDial. jib underlip, mouth, face, verb draw down the mouth, NE. jib foresail. Compare "the cut of one's jib" personal appearance; countenance.

8A8. Swiss röre vorgestülpte Lippen. Cf. 151B1.

8A9. Bav. schippe Schüppe; aufgeworfene Unterlippe. Cf.7H13.

8B1. EDial. gib upper lip of a fish, beak of a male salmon; hook; bent end of a stick; bend in a stream, OFr. gibe sorte de serpe. Cf. 8A7.

8B2. EDial. gam lips. Cf. 19B1a.

8C1. Swiss pampel grosse geschwollene Lippe, Hängelippe, bambel etwas Baumelndes; Hängemaul, NHG. bammeln, pampeln herabhangend hin und her schwanken. Cf. 127F1.

8C2. EDial. choller hanging lip of a dog. Cf. 14A11.

8C3. EDial. fipple underlip; pouting lip; wry face; anything loose, flaccid, hanging from the nose; crest of a turkey when elated; projecting, hanging part of a sheaf. Cf. Icel. flipi underlip of a horse (NED.). Compare Arnl. 33:10.

8C4. Bav. flarre Lippe. Cf. $7 \mathrm{~K} 2$.

8C5. Swiss lämpe herabhängende Unterlippe. Cf. 140A8.

8C6. EFris. leb, lebbe thick, hanging lip, MLG. lebbe underlip, LG. labbe Hängelippe, Swiss laps, lappelen, lippel hanging lip, NHG. lappen niederhängendes weiches Stück wovon. Cf. 7K10, 14A17, $22 \mathrm{~A} 1,85 \mathrm{~B} 5$.

8C7. MLG. lobbe, lubbe dicke hängende Lippe, Als. luppe grosse aufgeschwollene Lippe. Cf. 15A3.

8C8. EDial. lolly, loll lip. 22A4.

8C9. Lothr., Swab. lätsch big, hanging underlip. Cf. 7K5.

8C10. Steir. lesche Lippe, Unterlippe; plumper Mensch ohne Stärke und Mut, Swiss lasche Lappen, Streifen. Cf. 6H1, 7K6.

8C11. Swiss latz Ausschnitt am Kleide, der sich wenden lässt; klappenartiger Kleidungsteil; etwas Vorragendes, Vorstehendes; Unterlippe als Hängemaul, OFr. laz Schnürband (Wg.). Cf. $7 \mathrm{~K} 7$.

8D1. Swab. blätsche grosses, ganzrandiges Blatt; überhaupt eine bedeutende Fläche; grosse Lippe, Swiss blatsche grosses breites Blatt. Cf. 7H2.

8D2. Swiss lammele Messerklinge; Schoten der Hülsenfrüchte; verzogene Lippen, Fr. lamelle metal plate (Schw.). 
8E1. Als. ranft, ramft blaue Lippen eines Kranken. Cf. 7L1.

8F1. Steir. paper Lippe, Swiss pappen Papp machen; essen, Sax. pappelen mit zahnlosem Munde sprechen, undeutlich sprechen. Cf. 7A2.

8F2. Swiss läff Lippe, Bav. leffel idem, Swab. laffen schlürfen, lecken, MHG. idem. Cf. 7C11.

8F3. Als. schnubel grosse Lippe, schnuben schnauben. Cf. $9 \mathrm{~L} 17$.

\section{Nose, Snout, Beak}

The nose, snout, and beak are described (A) as a swelling, lump, chunk, knob, stump, bunch, and the like; (B) as a point, dagger, peak, horn, spout, angle, handle, edge; (C) as something elongated and round, as a tube, snail; (D) as a flat object; (Dd) as a hasp; (E) as a hook, crook; (F) as a funnel; (G) as an opening; (H) as that which is in front, such as a foresail, head; $(J)$ as a sheath; (Jj) as the external contour; (K) as the source of mucus; (L) as that which snorts, sniffles, "slorps," snores, gurgles, grunts, growls, gabbles, chirps; (M) as that which smells; (N) as that which eats; (O) by a proper name; (P) unexplained.

9A1. Swiss ber Beere; dicke Nasenkuppe. Cf. 51D2.

9A2. Swab. blunze Blutwurst; dicker kurzer Mensch; unförmlich dicke Nase; schwerer, fetter Körper, NE. blunt thick, etc., OE. Blunta man's name. Wood, Mod. Phil., XI, 330. Cf. 85A39, 117A1.

9A3. Swiss bölle dicke Nase. Cf. 26E1.

9A4. DuDial. poen fat person; fat, plump child; thick nose, poensjel large vat.

9A5. Als. pfumpf Stoss; knorriger Auswuchs; dicker kleiner Kerl; kleine, kugelige Nase, gepfumpft gedrängt voll, SwedDial. pampen aufgedunsen. Cf. 85A38, Arnl. 129:26.

9A6. Swab. fink Vogel; einen fink haben eine rote, erfrorene Nase; aus Tuchenden gemachte Schuhe, OHG. finko Fink. Cf. $123 \mathrm{~L} 1$.

9A7. Egerland gimpl rote Nase, Nase, NHG. gimpel Blutfink. Cf. 9A6.

9A8. Als. kide Schossling des Knoblauchs; Kiel eines kleinen Schiffes; Nase, Swiss chīd Keim, Schoss, Stengel; Bläschen auf der Haut; kleines Mass. Cf. $123 \mathrm{C} 19$. 
9A9. Swiss chlobe, Cologne klovve nose. Cf. 50A10.

9A10. EDial. knob nose. Cf. 2 A27.

9A11. Prus. knagge Pflock; hölzerner Wirbel an Tür und Fenster; Stück von ziemlicher Grösse; Nase.

9A12. Als. kübel grosse Nase, NHG. kübel tub; hod.

9A13. Swab., Als., Eichstätt. kumpf Gefäss in der Mühle; dicke grosse Nase, kumpf stumpf, Eichstätt. kumpl grosse, etwas gebogene Nase; hoher Hut.

9A14. EFris. kulpe, kulp dicke Tonkugel; dickes rundliches kolbiges grobes ungeschlachtes oder knolligtes, klumpiges Etwas; ungeschlachte und unförmliche Nase, Thur. kulpe dicke Nase. Cf. 26E2.

9A15. NE. conk nose, conch shell; domed roof of a semicircular apse, Fr. conque shell. Cf. 2A17, $25 \mathrm{D} 8$.

9A16. EDial. doup nose. Cf. 90A27.

9A17. Thur. trumpe Nase, Swab. traubenartiges Büschel; ein Ganzes von mehreren Dingen. Cf. 127A25.

9A18. Als. zolge grosse Nase. Cf. 5E6.

9A19. Kärnt. rienggl Schnauze, Rüssel des Schweines; grosses langes Stück, Als. ranke grosses, unförmliches Stück, Swiss runggel Rübe, NHG. runkelrübe.

9B1. Als. beck lange Nase, bick Schnabel; grosse Nase, Fr. bec bill; mouth; objects like a bill.

9B2. Swiss pfal Pfahl; dicke, unförmliche Nase.

9B3. Als. pfeif Pfeife; Nase. Cf. 123D1.

9B4. EDial. pecker bird's bill; human nose.

9B5. EDial. chep tip of the nose, NE., ONF. piece which holds the plowshare.

9B6. EDial. coulter nose, cooter-snoot long nose, NE. coulter iron blade fixed in front of the share in a plow; knife, OE. culter coulter.

9B7. Sax. gieke Messer; Säbel; Nase, gieks dolchartiges Messer, Als. gix altes stumpfes Messer.

9B8. Steir. giel Rüssel; Mund; Ausgussrohr; Schlund, gielen die Nase überall hineinstecken, MHG. giel Maul. Compare Arnl. $26: 27$.

9B9. EFris. gäfel Giebel; Nase, Fris. gevel idem, Prus. giebel idem, NHG. giebel. 
9B10. DuDial. gaffel nose; fork, NHG. gabel.

9B11. Lothr. gogel Nase, Swiss gugel spitzige Mütze; Hügel; runder Gipfel.

9B12. Sax. gurke Nase, Prus. grosse Nase, NHG. gurke.

9B13. Schles. heft, Egerland hiaft Handhabe; Nase. Cf. $123 \mathrm{~B} 10$.

9B14. EDial. horn nose; spout on vessels, MLG. damp-horn Löschnapf; Habichtsnase (dampen ersticken), Thur. lösch-horn Löschhorn; grosse Nase.

9B15. Prus. dulk, Thur. dulke Nase, LG. dulk Dolch; Mensch mit langer spitzer Nase.

9B16. Schles. titte Zitze; Schnabel, NE. tit. Compare Arnl. $101: 24$.

9B17. Prus., Sax., Eichstatt. zinken Nase, NHG. hervorstehende Spitze; lange Nase. Cf. 7 J7.

9B18. NE. sword the prolonged snout of a swordfish or sawfish.

9B19. Cologne nöll, Thur., Hess. nülle Nase, Swab. nolle rundlichter, harter Körper, dicker Mensch. Cf. 123C24.

9B20. Prus. nui Nase, from $n(i f f)+(h) u i$ both nose, OE. nebb. Compare Arnl. 23:02 and the following words.

9B21. LG. nuge nose, nugen schwanken.

- 9B22. Prus. hui nose, huien spotten, huijanen gähnen.

9C1. Bav. schlauch Rüssel des Elephanten. Cf. 123E5.

9C2. Bav. schnickel Rüssel; Schnauze des Hundes, NHG. schnecke. Cf. 123E7.

9D1. EDial. fluke a duck's bill; flat fish, OE. flóc flat fish, OHG. flah flach.

9Dd1. Thur. haspen Nase, NHG. haspe.

9E1. Swab. hap gekrümmtes Messer; gekrümmte Nase, Adlernase, häpe gekrümmtes Messer, MHG. hepe idem.

9E2. Steir. breinsabel Vogelschnabel; Nase, brein Brei+säbel.

9E3. Als. pandurensabel Pandurensabel; krumme, gebogene Nase, pandur ungarischer Soldat.

9F1. DuDial. domper conical extinguisher; thick nose with wide nostrils, dompen to extinguish.

9F2. Oberh. dutte Schnauze, Nase; Düte, NHG. düte trichterförmig gerolltes Papier. Cf. 7J4a. 
9G1. EDial. goner pig's snout, gone, gane gape, gan mouth, throat, jaws, ON. gana gape. Compare Arnl. 43:27.

9H1. DuDial. fok nose, Du. fok Fock, Focke.

9H2. Sax. täte Nase; Gesicht, Fr. tête head; top part; front part.

9J1. Lux. schek Hülse, Schote; Scheide; grosse Nase, Fr. jaque jacket, NE. jacket garment; natural covering of animals; skin of potatoes.

9Jj1. EDial. shape nose; corn without kernel, NE. external form, contour; mold. For different meanings compare Arnl. 140:01.

9K1. Cologne rotzpatrie Nase.

9K2. Sax. rotzstute Nase. Cf. 9F2.

9K3. EDial. snotter nasal mucus; nose, DuDial. snotje nose, snotterik snout of a turkey. Cf. 10D1.

9L1. DuDial. gorre large nose, MDu. gorren grunt. Cf. 9L8.

9L2. Prus. kautsche Schnauze. Cf. 7D19.

9L3. Westf. kijack Schnabel. Cf. 41D3.

9L4. EFris. käkel Schnauze. Cf. 7D22.

9L5. Swab. kipp Schnabel; Wortgezänk; Hund, Sax. kippeln keifen, zanken, Du. kibbelen zanken.

9L6. Thur. kulke fliessende Nase, kulken unter gurgelndem Geräusch trinken, auch vom Geräusch der Eingeweide, Sax. gulken vom Geräusch beim Auslaufen des Wassers aus einer Flasche.

9L7. Swiss läff Schnauze. Cf. 7C11.

9L8. MDu. morre, Swiss, Kärnt. murre snout, MDu. morren grunt, NHG. murren.

9L9. Swab. mufti Nase, komische Bez. statt muffer Maulhänger, muffen murren, knurren (F.).

9L10. Westf. nüesel Lichtschnuppe; Nasenschleim; Nase. Cf. $10 \mathrm{~B} 1$.

9L11. Swiss, Thur., Sax. nüschel Schnauze. Cf. 2K1.

9L12. Du. snater, Westf. sneter beak. Cf. 7D36.

9L13. Flem. snierk cow's nose, Du. snorken snore.

9L14. EDial. snirl nose, verb snarl, NE. snurl nostril, WFlem. snorrelen snuffle, snort. Cf. 10B2.

9L15. Prus. schnorgel Nase, Schnauze. Cf. 7D39.

9L16. Als. schnuffel Schnauze, Rüssel, NE. snuffle. Cf. 6C5, $7 \mathrm{C} 7$. 
9L17. Als. schnuber Nase. Cf. 8F3.

9L18. Bav. schnauppen Schnabel, Schnauze, Mund, MHG. snüben schnauben, schnarchen. Cf. 7D35a, 8F3.

9L19. Kärnt. schnüder Nase, schnudern den Atem durch die verstoppte Nase ziehen, Lux. schnudelsack Rotznase, MHG. snüden schnarchen, schnauben. Cf. $123 \mathrm{E} 8$.

9L20. Als. schnurre Schnauze, Rüssel. Cf. 7D38.

9L21. Ruhl. schnüss Nase, Lux. schnöss Schnauze, LG. snuss Schnauze, Nase, Rüssel, Westf. snüssel Rüssel, Wald. snüsel idem, LG. snūsen schnauben, schnüffeln. Cf. 7C8.

9L22. Du. slurf, Fris. sloarf elephant's snout, NHG. schlürfen.

9L23. DuDial. tjibber nose, EDial. chipper chirp, subst. the crossbill.

9L24. DuDial. plat-sjirp flat nose, sjirpen chirp, NE. chirp.

9M1. Lux. richel nose, Sax. riecher, riechhorn, riechkolben, Ruhl. richer, Prus. riecher, LG. rīker, EFris. ruker nose, NHG. riechen.

9M2. Als., Steir., Lothr., Bav. schmecker nose, schmecken riechen.

9M3. NE. smeller nose.

9N1. Lothr. fross Frass; Rüssel, Lux. frōss idem. Cf. 7A3.

901. Swiss kölliker eine unförmlich grosse Nase, von einem Mann, der jenen Namen trug und durch jene Eigenschaft allgemein bekannt war.

9P1. EDial. gongar large nose.

\section{Nostril}

The nostril is described (A) as a hole; (B) as that which snuffles, smells; (C) as a wind catcher; (D) as a source of mucus.

10A1. NE. blow-hole nostril of a cetacean.

10A2. Du. ademgat nostril. Cf. 42B1.

10A3. Swiss chämin, Swab. kamin nose. Cf. 7F12.

10A4. MDu. loc hole; nostril. Cf. 7F16.

10A5. MDu. snotgat nostril, nose.

10A6. EDial. thirl hole; nostril, OE. pyrel hole, etc. (Wr.).

10B1. Fris. noaster, EFris. nöster, NHG. nüster, MLG. nuster, DuDial. noster, nöster nostril, MLG. nuseln, nüseln leise und undeutlich reden, schnüffelnd wühlen. 
10B2. EDial. snirl nostril. Cf. 9L14.

10C1. Sax. windfang Nase.

10D1. EDial. snotties nostrils. Cf. 9K3.

\section{ChIN}

The chin is described (A) as something protruding; (B) as that which gabbles, mumbles.

13A1. Bav. bart beard; chin (also of women), Als. idem, Nös. idem.

13A2. DuDial. bef sharp, projecting chin. Cf. 8A2.

13A3. EDial. gash chin; projection of the underjaw, adj. protruding, verb protrude.

13A4. Swiss götsch, gösche Kinn. Cf. 7J2.

13A5. EDial. chinkie from chin. Cf. 14A9; Arnl. 35:03.

13A6. DuDial. knep big projecting chin, Flem. club, stick.

13B1. Lothr. gäbber Kinn. Cf. 7D14.

13B2. Swab. maffel Kinn, mäffen murren. Cf. 7D27.

\section{Double Chin, Dewlap, Wattle}

The double chin, dewlap, and wattle are described (A) as something swollen, a flabby mass, flap, sack, etc.

14A1. Swiss ingeli Zäpfchen am Halse ungehörnter Ziegen. Von singele ('s singele $>$ 's ingele) Ohrfeige, Bav., Austr. singese Klingel (Schw.).

14A2. Swab. bummel Halslappen am Rindvieh. Cf. 123C8.

14A3. EDial. fyte-poke double chin, flyte scold + poke bag (Jam).

14A3a. Du. wam, NHG. wamme dewlap. Cf. $17 \mathrm{C} 1$.

14A4. Thur. wappich Doppelkinn, Wamme, Sax. wappig Schmerbauch, MHG. wappen in schwankender, zitternder Bewegung sein.

14A5. NE. wattle bag, wallet; fleshy lobe hanging from the front of the head, OE. watel wattle, interwoven twigs. Cf. 5E4, $25 \mathrm{D} 4$.

14A6. Bav. troller fleischiges Unterkinn, Swab. rundes Holz; kurzer Sägblock, trollen rollen, wälzen.

14A7. Swiss zötteli wattle, NHG. zotte herabhängender aus Haaren oder Faden bestehender Flausch, Du. todde Lumpen, Fetzen.

14A8. Hess. schwulch Geschwulst; Doppelkinn, NHG. schwellen. 
14A9. LG. kinkel Doppelkinn; Fettwürfel; hangendes Stück Fett, NE. kink. Cf. 13A5, 123A16.

14A10. MDu. coder dewlap, MLG. koder double chin, Bav. koder idem, ON. koðre scrotum, OE. codd bag, shell, husk. Cf. 44C1, 101A10.

14A11. EDial. choller dewlap, double chin, wattles of a turkey cock, OE. ceolur throat. Cf. 8C2, 16A1; Arnl. 43:34.

14A12. Du. kossem dewlap, double chin, Norw. kusma mumps, Ger. $k u p-, k w e b-$ in ON. kvipr Bauch, Goth. qipus Bauch, Mutterleib (Franck).

14A13. DuDial. kwabber dewlap, Du. halskwabbe idem. Cf. $15 \mathrm{~A} 1$.

14A14. DuDial. kwats double chin; hanging swelling, Flem. fat, heavy body; swelling.

14A15. Steir. klankerl Zäpfchen am Halse, beim Geissvieh, Swiss orenchlänker idem, chlanker Glockenschwengel. Cf. 24B2.

14A16. Swiss lämpe dewlap, double chin, Als. dewlap. Cf. $140 \mathrm{~A} 8$.

14A17. EFris. leb dewlap, NE. dewlap. Cf. 8C6.

14A18. Du. lel flap under the beak of some fowls. Cf. 22A2.

\section{Cheek}

The cheek is described (A) as a swelling, lump, or rounding object; (B) as that which munches.

15A1. EFris. kwabbe, kwab alles, was aufgeschwollen und weich, elastisch und nachgiebig ist und durch Berührung leicht in Bewegung gerät; Wange; eine weiche sumpfige oder morastige Stelle im Lande, Westf. kwabbel fetter, hervorschwellender Körperteil, DuDial. flabby mass. Cf. 7H15, 14A13, 140A5.

15A2. MDu. lier cheek, Du. idem, MLG. lēr idem. Cf. 85A34.

15A3. EFris. lobbe, lob weiche schwammige oder schlaff niederhängende, dick aufgeschwollene Fleisch- oder Fettmasse; Wange, NE. lob country bumpkin; something pendulous; lump, large piece, MLG. lobbe lump. Cf. 8C7, 74B13.

15A4. Swab. malle dicker, starker Mensch; dicke Backen, molle dicker, aufgedunsener Mensch; Ochs, mallig (of new-mown hay) ballt sich. 
15A5. EFris. kōn cheek, MDu. coon, Du. koon idem, ON. kaun boil, swelling. Cf. $23 \mathrm{~A} 2$.

15B1. Swiss munze, monze Wange; Kuss, munzen mühsam kauen, Swab. muntschen undeutlich reden; essen.

\section{GILL}

The gill is described (A) as a swelling; (B) as a flap; (C) as a slit, cleft.

16A1. EDial. choller gills. Cf. 14A11.

16A2. DuDial. jotje gill. Cf. jottes the curving sides of a ship.

16A3. DuDial. koen gill. Cf. 15A5.

16B1. DuDial. wam gill. Cf. $17 \mathrm{C} 1$.

16C1. NE. gill organ of respiration of water-breathing animals; Swed. gäl, Dan. gjaelle agree in meaning but do not explain the form of the English word (NED.). Compare Arnl. 45:02; Norw. gil cleft, rent in the mountains.

17. JAW

The jaw is described (A) as that which cracks, crunches, munches, mumbles; (B) as a swelling; (C) as a flap; (D) as the under part of the mouth.

17A1. EDial. chanks under part of a pig's head, chank chew, bite, chanks, chankings scraps, bits, fragments, NE. chank apparently var. of champ to munch (NED.).

17A2. EDial. chaps, chops jaw; cheeks; pig's cheek, NE. jaw; part of the bill of a bird, verb crack; strike sharply.

17A3. NFris. kentjāp Kinnbacken, Unterkiefer; Gebiss, ken chin. Probably identical with or related to preceding.

17A4. Westf. knaschen Kinnbacken; Gebiss eines Hundes, knatschen hörbar essen, Norw. gnaske langsam mit knackendem Laute kauen.

17A5. Steir. mummel Kinnlade. Cf. 7D29.

17B1. MDu. coon jaw. Cf. 15A5.

17C1. DuDial. wam jaw, Du. lobe; opened belly of a fish, NHG. wamme belly, womb, etc. Cf. 16B1, 14A3a; Arnl. 103:12.

17D1. Du. onderbek lower jaw, Fr. bec beak.

17D2. Du. ondermuil, Als. undermul lower jaw. 
18. Gums

The gums are described (A) as mumblers; (B) as protectors of the teeth.

18A1. DuDial. barbels toothless gums, NHG. barbelen bappeln, blaterare.

18A2. Du. babbelen toothless gums, NE. babble.

18A3. Du., Fris. kevel gums, MHG. kifen nagen, kauen. Compare Arnl. 40:10.

18A4. Fris. gavels gums, OE. geaflas jaws. Compare Arnl. $40: 13$.

18A5. MDu., MLG., Du., EFris. gagel gums, palate, OE. geagl jowl, jaw. Compare Arnl. 40:14.

18B1. NFris. hul gums, Fris. husk, shell.

18B2. NHG. zahnfleisch gums.

\section{9. Тоотн}

The tooth is described (A) as that which bites, grinds, hacks, cracks, nicks, nips, pulverizes, cuts; (B) as to the shape as something crooked, flat, sharp, pointed, hanging; (C) in reference to the eyetooth, as a hook, the eyetooth, the corner tooth; (D) in reference to the first teeth, as caps, from their position and the fact that they come out, or as milk teeth because they are used during the sucking period; (E) in reference to all the teeth together, as a collection of ivory, of bones, a battery, a honeycomb; $(\mathrm{F})$ in reference to a new tooth, as a swelling, lump; $(\mathrm{G})$ with reference to the root, as having branches, prongs.

19A1. DuDial. bieter tooth, Swiss bīsser idem, bissen bite.

19A2. NE. grinder that which grinds; molar, tooth.

19A3. Swab. hackerle Zahn, hackerlich Zähne, NHG. hacken.

19A4. Swab. krächling Nuss; Zahn, NHG. krachen.

19A5. EDial. neg, neggy a baby's tooth, nag to nick; bite; mark with the teeth, subst. bite; nick.

19A6. NE. nipper one who nips; pincer, tong; incisor tooth, nip to pinch.

19A7. Swiss raffle Reibeisen; grober Kamm; Gebiss, rafflen zerreiben. 
19A8. NE. razor instrument for shaving; tusk of a boar, OFr. rasoir razor (CD).

19B1. EDial. aikle molar tooth. Cf. aigle-tooth sharp, pointed tooth, aigle (<Fr. aiguille needle [Wr.]) icicle.

19B1a. EDial. gam, gamb tooth; mouth, adj. overlapping (of teeth), awry, game, gammy lame, deformed, crooked, Fr. gambi bent (Wr.). Cf. 8B2.

19B2. Swab. lälle Zahn. Cf. 22A2.

19B3. Swiss pale Schaufel; Ruderblatt; Schneidezahn des Rindviehs, Ital. pala shovel, ladle, oarblade (Schw.).

19B4. EDial. peg tooth, esp. of a child, NE. peg. Cf. 69 B5.

19B5. NE. snag sharp protuberance; short projecting stump, etc.; the fang or root of a tooth, Icel, snagi peg (CD).

19B6. EDial. tuck, tuxes tusk, probably abstracted from tux (as if $p l$.), OE. tux, tusc tusk, NE. tuxes $p l$. (Wood). Compare Arnl. 32:01. From Fr. estoc (NED.).

19B7. Als. stupfel Stoppel; zerbröckelter Zahn. Cf. 35D2, $62 \mathrm{~F} 3$.

19C1. NHG. haken hook; eyetooth, Du. haaktand idem.

19C2. Du. oogtand, NE. eyetooth.

19C3. EDial. week-tooth canine tooth, wick corner, angle (Wr.).

19C4. Du. hoektand eyetooth, hoek corner, angle.

19D1. Swiss chappe Mütze; Milchzahn des Rindes.

19D2. NE. milk-tooth, NHG. milchzahn first tooth.

19E1. Swab. klavier wie Nhd.; Gebiss. Cf. 53D1.

19E2. Oberh. geriffel Zahnwerk im Munde. Cf. 175A2.

19E3. Prus. hotzbatterie die beiden Zahnreihen, hotzen wiegen; tanzen, Swab. zittern, schwanken. Cf. 6S1.

19E4. Hess. mārt all the teeth; jaw teeth, EFris. mārt honeycomb.

19F1. Sax. wölflein junger Zahn, Bav. Zahnbeule; Zahn, NHG. wolf lupus; Hautentzündung.

19F2. EDial. tut tooth; hassock, footstool; small pad, NE. tutty tuft or bunch of flowers, adj. hummocky, tutty-nosed snubnosed. Cf. 90A56.

19G1. Tirol. grī $f$ Zahnwurzel; Zweig, ON. greip space between thumb and fingers. Compare Arnl. 56:03. 


\section{Enamel}

The enamel of the teeth is described as something gleaming.

20A1. Swiss gläste Glanz; Glasur, Zahnschmelz, glästen glänzen.

20A2. NHG. zahnschmelz enamel of the teeth, schmelz Glasur der Zähne; weiche Glanzfarbe, ON. smeltr emailliert.

\section{Palate}

The plate is described as a vault. DuDial. hemel sky, heaven; palate, NHG. himmel.

\section{TONGUE}

The tongue is described (A) as something hanging, a flap; (B) as something sharp, pointed; (C) as a blade; (D) as that which licks; (E) as that which puffs, gabbles, clappers, cries, "slorps"; (F) contemptuously, as it hangs from the mouth as a picture, something gleaming, a roof tile.

22A1. DuDial. leeren lappe protruding tongue, Fris. sūchlape Sauglappen; tongue.

22A1a. Oberh. löffel Ohren des Hasen; Zunge des Hirsches, NHG. löffel. Compare Arnl. 20:04.

22A2. Du. lel movable pellicle; tongue; flap, Swiss, Swab. lälle ausgestreckte Zunge; Eiszapfen, Als. lälli Zunge, Swiss lällen die Zunge herausstrecken; die Lippen ablecken; auflodern. Cf. 7K8, 14A18, 19B2, 81C6.

22A3. DuDial. lerp tongue; whip, lash, Du. larp whip, switch.

22A4. EDial. loll, lolly tongue, NE. loll to hang down loosely; droop, dangle. Cf. $8 \mathrm{C} 8$.

22B1. Du. angel snake's tongue, NHG. fishhook; bee sting, etc.

22B2. EDial. stinger snake's tongue. Cf. $92 \mathrm{~K} 1$.

22B3. EDial. spill splinter; wooden peg; tongue.

22C1. MLG. blat Zunge, MDu. leaf; tongue, DuDial. blad, blat, blare (*bladere), bledder tongue, NE. blade.

22D1. Als., Swab. lecker vorderer Teil der Rindzunge, NHG. lecken.

22E1. Fris. blaeijer tongue. Cf. 7D3.

22E2. EDial. clack, clacker sudden recurring sound; woman's tongue, NE. clack sharp, sudden, dry sound. Cf. $42 \mathrm{C} 2$. 
22E3. EDial. clapper wooden rattle; cymbals; talkative person's tongue, Flem. klep, klepel, Antwerp klep tongue. Cf. 7D23.

22E4. Cologne krischting Zungenstück beim Rindvieh, Lux. krischen kreischen, krischer Schreihals.

22E5. Flem. knibbel tongue. Cf. 7D24.

22E6. Flem. slabbaris tongue. Cf. 7C3.

22E7. DuDial. slerf tongue, early Du. slorven sorbere, Lor. schlurben schlürfen.

22E8. DuDial. slerp tongue, slorpen "slorp," EDial. slorp, NHG. schlürfen.

22F1. DuDial. beeldeken, beleken devotional picture; tongue, hij steekt zijne tong uit en zet een beeldeken.

22F2. Swab. blecke Zunge von Tieren, blecken sehen lassen, OHG. blicchen glänzen; blicken.

22F3. Sax. dachziegel Zunge.

\section{TonsiL}

The tonsil is described as a swelling, an almond-shaped, beadlike object.

23A1. Du. amandel tonsil, EFris., NHG. mandel idem, OHG. mandala almond from Lat. amandula.

23A2. DuDial. koen tonsil. Cf. 15A5.

23A3. DuDial. kralen tonsils, OFr. coral, MLat. corallus coral (Franck).

\section{Uvula}

The uvula is described (A) as a blade; (B) as a swelling, lump; (C) as a hook, crook; (D) as that which clucks; (E) as something within.

24A1. NHG. atemblatt uvula, Swab. hauchblatt idem. Compare Arnl. 31:02.

24B1. WFlem. hijf uvula; inflammation of uvula, sometimes mistaken for a tuft of hair growing in the throat and to be cut out, NE. hives any eruption on the skin when the disorder is supposed to come from an internal cause, red gum, chicken pox, nettle rash; inflammation of the larynx (origin uncertain, NED.).

24B2. EDial. clunker uvula. Cf. 123A18. 
24C1. Du. huig, Dial. hoek, MLG. hūk, Prus. huke, EFris. hük, NHG. hauch uvula, Ger. *hüka (Franck), Swiss hauchen Haken; gekrümmter Griff am Sensenstiel.

24D1. EDial. clock uvula. Cf. 101C2.

24E1. DuDial. zieltje uvula; shoe tongue, ziel soul; inmost part. Cf. $117 \mathrm{C} 1$.

\section{EAR}

The ear is described (A) as to its function, as that which listens, hears; (B) as to the interior, as a drum; skin; blade; (C) as a tube, passage; stick; (D) as to its exterior, as something hanging, a flap, end, edge; a swelling; a funnel.

25A1. Thur. horch Ohr, NHG. horchen.

25A2. Steir., Swiss, Swab. loser Horcher; Ohr, losen horchen.

25B1. NE. drum, NHG. trommelfell tympanum.

25B2. Du. oorvlies eardrum.

25B3. Swab. blatt Trommelfell, NE. blade.

25C1. Du. gehoorbuis tubes of the ear, buis tube.

25C2. Du. gehoorgang, -weg Eustachian tube.

25C3. Swab. gehoerstock inner ear.

25D1. Swiss orenlampli Ohrläppchen. Cf. 140B3.

25D2. Sax., Steir. ohrwaschl Ohrläppchen, Bav. waschl idem; Strohriegel zum Reinigen.

25D3. Swab. behenk was hängt; hangende Ohren der Hunde, NHG. hangen. Cf. 36A1, 116B1, 126B3, 81C2, $115 \mathrm{C} 1$.

25D4. EDial. wattle ear. Cf. 14A5.

25D5. EDial. tab narrow or pointed end; tatter; ear, tib ear. Cf. 91B3.

25D6. Kärnt. trichter wie hd.; Ohr.

25D7. Swiss chabisblatt grosse Ohren, chōlblatt idem.

25D8. NE. conch external ear. Cf. 9A15.

25D9. Als. läppel, NHG. ohrläppchen, Fris. earlapke, MLG. ōrlappe ear lobe, DuDial. lap hanging ear of a dog, Swiss geharschlappen herabhängendes $\mathrm{Ohr}$ der Kuh (geharsch stark behaart). Cf. 8C6.

25D10. Als. lafete Ohren: from l(appel) (25D9)+(l)afete. Cf. $6 \mathrm{C} 4$. 
25D11. DuDial. lodje ear lobe, lodsoor hanging ear, lodder flap, tatter.

25D12. EDial. lug ear; handle of a cup or pitcher; knot, tuft; tassel, flap; head of a shepherd's crook, etc. Cf. lur child's word for ear: from $l u(g)+(e a) r$.

25D13. NE. list border, edging, strip; fillet; lobe of the ear; ear, OE. līste border of cloth. Cf. 5E13.

\section{EYE}

The eye is described (A) as that which winks, peeps, looks, glares; (B) as that which gleams; (C) as that which sleeps; (D) as a window, opening; (E) as a sphere, ball; (F) as a disk, wheel; (G) as something protruding; $(\mathrm{H})$ as to the retina, as veined or granular skin; $(\mathrm{J})$ as to the tear glands, as the fleshy part; $(\mathrm{K})$ as to the lid, as that which blinks, a lid, flap.

26A1. Lothr. a-keiken ( $a=e i n)$ Augen, Prus. kicker Auge; Zuschauer; Fernrohr, Fris. kikert eyes, Wald. kīken idem, LG. kīken gucken.

.26A2. NE. winker eye. Cf. 29A3.

26A3. Bav. glorren Augen, Steir. glur Glotzauge, Kärnt. glurre grosses stieres Auge, Prus. glarren Augen, NE. glare.

26A4. Schles. glotzen Augen, Swab. glotzer idem, glotzen starr blicken, NE. gloat.

26A5. Swab. gucke, gucker, Als. guckel, Lothr. guggel, Swiss gugger, Lothr. gickle eye, NHG. gucken.

26A6. Als. lueger, Swiss g'lueg Augen, OHG. luogēn aus einem Versteck hervorsehen, lugen.

26B1. Bav. birkling, berkaug, birgaug Auge welches ganz weissgrau oder weisslicht ist; Auge überhaupt. Etwa zu birke, in Bezug auf das Weiss der Rinde (Schm.).

26B2. EDial. glorys eyes, NE. glory renown; nimbus.

26B3. Swab. leuchte, Swiss liecht Auge, NHG. leuchten.

26C1. Thur. lunze Auge. Cf. $7 \mathrm{~K} 9$.

26D1. EFris., Swiss fenster Auge.

26D2. DuDial. kot eye. Cf. 28A1.

26E1. Swiss böllen grosse Augen; bollen etwas Kugeliges. Cf. 2A3, 9A3, 68A2, 90A8; Arnl. 142:17. 
26E2. Wald. kölpen Glotzaugen, LG. külpen unreifes Obst; grosses Auge, NHG. kolpe kolbiges Ende. Cf. 9A14.

26E3. Swab. knäller grosses, hervorstehendes Auge. Cf. 117A3.

26E4. Sax. schusterleuchtkugeln grosse Augen.

26E5. NE. orb globe, ball; eye, Lat. orbis circle, etc.

26E6. Als. troller, truller Gegenstand zum Rollen; grosse Augen, trolen rollen, kugeln. Cf. 5B3a.

26F1. Als. pfluegsrädle Pflugrädchen; Glotzauge.

26F2. Swiss schībe Scheibe, etc.; Auge.

26G1. Tirol. grūl wide-open, protruding eyes. Cf. 145D1.

26H1. Du. adervlies retina.

26H2. Du. druifvlies third eye membrane, druif Knopf; Traube.

26J1. Swiss augenfleisch Tränendrüse, im innern Winkel.

26K1. Swab. blickel Fensterladen; Augenlid, NHG. blick.

26K2. Lothr. a'endeckel, Swab. augendeckel eyelid.

26K3. Bav. augenhöbel eyelids, hobel Decke.

26K4. Swiss lid, NE. lid cover; eyelid.

26K5. MDu. schedel, Du. oogschedel eyelid, schedel deksel.

26K6. Bav. augengleff eyelid, gleff labium. Cf. 8F2.

26K7. Lothr. lechel eyelid, Lux. lichel idem, lichen to lift.

\section{PUPIL}

The pupil is described (A) from the small image reflected therein, as a little child or bird; (B) as a hole; (C) as that which gleams.

27A1. NHG. kindlein, Zwickau khinl Kindchen; Pupille.

27A2. EDial. bird pupil.

27B1. Prus. augenloch Pupille.

27C1. EDial. shine pupil, verb show a light.

27C2. EDial. star pupil; speck in the eye.

\section{Eye Socket}

The socket of the eye is described as a hole, pit, recess, angle.

28A1. DuDial. kot hole; eye socket. Cf. 26D2.

28A2. MDu. ogeput, -pit (NE. pit), Du. oogkuil (kuil hole), Du. oogholte (holte hole) eye socket.

28A3. Du. winkel corner, angle; eye socket, NHG. winkel. 


\section{Eyelash}

The eyelash is described (A) as a winker; (B) as something flexible; (C) as the hair of the eye or lid; (D) as something woolly, feathery.

29A1. Cologne augeflitter Augenwimper, fittern sich unstät hin- und herbewegen.

29A2. Du. pinkers eyelashes, DuDial. pinkhaar idem, pinken to wink, twinkle.

29A3. NE. winkers, eyewinkers eyelashes.

29A4. NHG. wimper eyelash, OHG. wint-brāwa. Compare Arnl. 12:02.

29B1. Fris. eachteister eyelash: each eye+teister sprout of a plant.

29B2. NE. lash flexible part of a whip; eyelash, NHG. lasche angesetzter Streifen, MLG. las, lasche keil- oder zwickelförmiger Streifen.

29C1. DuDial. scheelhaar eyelash, scheel cover.

29C2. DuDial. ooghaar eyelashes.

29D1. Bav. augenflanl Augenwimper, NE. flannel an open woolen stuff; certain woolly-leaved plants.

29D2. DuDial. vlegger eyelash, vlag grass-grown, heath-grown turf.

29D3. Flem. plomp, plump eyelashes, SLimb. plumpe idem. Probably from pluimpje little plume.

\section{Eyebrow}

The eyebrow is described (A) as a border; (B) as something furry. 30A1. Swiss bram Augenbraue; Rand, Kante, Bav. bräm idem, NHG. brame, bräme Rand, Randbesatz.

30B1. Sax. miezchen Katze; kleine Federn; Fädchen, Fasern; Augenbrauen, NHG. Kätzchen.

\section{Temple}

The temples are called the brain from association therewith.

31A1. WFlem. hersen, hirsen the temples, Du. hersens idem. Compare Arnl. 2:02. 
32. Crown

The crown is described (A) as a whorl; (B) as a top, peak; (C) as a bush, bunch, protuberance, projection.

32A1. MLG. dwerl, twerl Wirbel, Locke, Du. dwarrelen twirl.

32A2. Prus. kreisel der hohle Treibkreisel; Haarwirbel auf dem Kopf; Wirbelwind, Lothr. kres Kreis; Scheitel, NHG. kreisel spinning top.

32A3. Als. strubel Scheitel, Wirbel auf dem Kopf; Verwirrung, Lothr. struwe Haarquirl auf dem Scheitel, MHG. strüben rauh emporstehen. Cf. 5D13.

32A4. NHG. wirbel, EFris. warvel, warpel crown; whirl. Compare Arnl. 3:14.

32A5. Swab., Swiss girbel Kopfwirbel. Cf. 2E4.

32B1. Lothr. kipp Scheitel. Cf. 2E6.

32C1. Als. buschel Büschel; Scheitel.

32C2. Swiss ros Rose; Haarwirbel. Cf. 91B1.

32C3. Als. burzel Scheitel, höchste Stelle des Kopfes; Kopf; Haarwirbel auf dem Rücken des Schweines. Cf. 90A19.

$32 \mathrm{C} 4$. EDial. cantle crown of the head. Cf. $69 \mathrm{C} 3$.

32C5. Swiss chamb Scheitel; Kamm der Hühner; Kamm; Bergrücken, EFris. kamm idem, NHG. kamm. Cf. 36C1; Arnl. 4:01.

32C6. DuDial. moet crown of the head; roughness on printed paper caused by the impression of the type, Du. moet little knob, etc.

\section{Parting of the Hair}

The parting of the hair is described (A) as a groove, cut, split, dividing; (B) as a strip; (C) as something gleaming; (D) as something slippery.

33A1. Lothr. grät Scheitel; Rinne im Boden, NHG. grat Spitze; oberster sich hinziehender scharfer Rand wovon, MHG. Spitze; Bergrücken, etc.

33A2. EFris. harklöfe Haar-Scheitel, klöfe split, notch.

33A3. Schles. krinne Einschnitt, Kerbe; Haarscheitel, MHG. Kerbe, etc.

33A4. Sax. lauseschnitt sorgfältig gezogener Scheitel. 
33A5. Swiss scheid Scheidung, Trennung; Scheitel, NHG. scheiden.

33A6. NFris. sjuarel Scheitel, sjuar Scheere.

33A7. Lothr. spall Spalte; Haarscheitel, spallen spalten.

33B1. DuDial. streep narrow strip; parting of hair, NHG. streifen.

33C1. DuDial. blesse parting of hair. Cf. 4A1.

33D1. Sax. lauseschuscher Scheitel; Glatze, schuschen gleiten.

\section{Crest}

The crest is described (A) as the summit, top; (B) as a pad, bundle, bump, protuberance, tuft.

34A1. NE. cop head or top, esp. of a hill; tuft on the head of a bird, copple bird's crest; little summit, OE. cop top, summit.

34A2. EDial. top tuft, esp. bird's crest, DuDial. topp idem, hen with a crest. Cf. $2 \mathrm{E} 3$.

34B1. Swab. butsch Federschopf. Cf. 5A4.

34B2. NHG. holle Federschopf. Cf. 2 G5.

34B3. Swiss huppe Federbusch. Cf. 5A39.

34B4. NHG. kaupe Federbüschel; Grasbüschel; kleiner Erdhügel, Du. kuif tuft, crest, comb, MG. kuppe Berggipfel.

34B5. Flem. kobbe crest, comb, feather tuft. Cf. 5A30.

34B6. Swiss, Als. kobel aufstehender Federbusch, Lothr. kowel idem, Du. kovel Mönchskappe, Haube. Cf. 5A31.

\section{BeARD}

The beard is described (A) as down; (B) as hair; (C) as a bunch, tuft; (D) as stubble; (E) as a point; (F) as a noisemaker; (G) as gnats.

35A1. Swiss bluest etwas mit Blüten Verglichenes; Bartflaum, MHG. bluost Blüte. Cf. 59D1.

35A2. Swiss flām leichter Anflug, Decke, von Schnee, Barthaaren im Gesicht. Cf. 81CC1.

35A3. NHG. flaum weiche Bauchfedern, erster Federwuchs; erster Bartwuchs; weiche Wolle am Obst, Lat. plūma small, soft feather. 
35A4. Swiss gauch, Swab. gauchhaar Flaumhaare von jungen Vögeln; Haar im Genick; Bartflaum, Als. gauchenhar Bartflaum. Gauch hair, abstracted from gauchhaar, gauch simpleton, fool.

35A5. Sax. meinzel, mäunzigen, meizel wolliges Blütenzäpfchen oder -kätzchen; Bartstoppeln, Swab. maunz Katze. Cf. $127 \mathrm{G7}$.

35B1. DuDial. fas beard, MDu. hair. Compare Arnl. 162:49.

35C1. Du., Fris. knevel beard on the upper lip; cat's beard; anything big and strong, NHG. knebel idem.

35C2. MLG. knōp Knoten, Knopf; Knospe; Knebelbart, OHG. chnopf Knotenartiges, NHG. knopf.

35C3. Sax. wischer Schnurrbart, NHG. wischen.

35D1. Swiss stroffelhaar struppiges Barthaar, stroffeln Stoppeln.

35D2. Als. stupfel Bartstoppel, NE. stubble idem. Cf. $19 \mathrm{~B} 7$.

35D3. DuDial. bakkebies beard, bies rush.

35E1. Als. schnauz, Cologne schnäuzer, Sax., Lothr. schnauzer, Kärnt. schnautz Schnurrbart, NHG. schnauze.

35E2. Flem. sik goat; chin beard, Du. sik idem, from NHG. zicke (Franck).

35E3. NE. goatee chin beard.

35F1. Sax. rasauner Schnurrbart, rasaunen ungestüm rennen, dahinrasen: a blend of ras(en)+(pos)aunen.

35F2. Lux. schnorres, Du. snor, NHG. schnurrbart. Cf. 7D38.

35G1. Rap. muke Mücke, Fliege; Barthaare unter der Unterlippe, Swab. mucke Mücke; Barthaare unter der Oberlippe.

\section{Mane}

The mane is described (A) as something hanging; (B) as prickles; (C) as a comb; (D) as a wreath.

36A1. DuDial. behang horse's mane. Cf. 25D3.

36B1. Swab. grat Mähne des Pferdes; Spitze, etc., MHG. grāt Spitze, Ährenstachel, etc. Compare Arnl. 109:01.

36C1. Swiss chamb Mähne; Halsrücken. Cf. 32C5.

36D1. Swab. kranz Mähne. NHG. kranz.

\section{Skull}

The skull is described (A) as a knob, knot; (B) as a plate, lid; (C) as a container, such as a cup, dish, etc. 
37A1. Thur. kolbe Schädel, NHG. kolben.

37A2. Thur. masel Maser, knorriger Auswuchs an Bäumen; Schädel, NHG. maser.

37B1. Swiss blatte Felsplatte, etc.; Schädelplatte, Ruhl. blatten Platte; Kopf.

37B2. Sax. deckel Hirnschale, NHG. deckel. Cf. $26 \mathrm{~K} 2$.

37C1. Du. bekkeneel skull, MDu. beckineel part of the head covered by the helmet, OHG. becchin, NHG. becken .

37C2. MLG. bregenvat skull.

37C3. MLG. bregenkop skull, OE. copp Becher.

37C4. Steir. pavesen-kammer skull. Cf. $2 \mathrm{~F} 1$.

37C5. Fris. panne pan; tile; skull, Du. pan, MLG. hernepanne, NHG. hirnpfanne, MLG. bregenpanne, Fris. breinpanne skull, NE. pan. Cf. 72A1.

37C6. DuDial. scheutel, schotel skull, MLG. schuttel Schüssel. 37C7. NE. skull cranium. Probably from Scand., ESwed. skulle forehead; originally shell. Compare Arnl. 6:13.

37C8. Westf. küls Schädel, Swiss chulle Grube, kleiner Behälter, Lothr. kul Vertiefung, Grube.

37C9. Swiss hafe Topf, Gefäss; Schädel, MHG. hafen Topf, Gefäss.

37C10. Swab. hirnhäuslein Schädel.

37C11. Swab. hirnkasten Schädel.

\section{Fontanelle}

The fontanelle is described as a fountain, as in the NE. fontanelle. 38A1. Swiss brunnen fontanelle.

\section{Brain}

The brain is described as something soft.

39A1. EDial. pash brains. Cf. 2M2.

\section{NECK}

The neck is described (A) as a swelling, chunk, lump, bump, knot, ridge; (B) as to the nape, as a hollow; (C) as a root; (D) as having spines; (E) as the part pierced (by the butcher); (F) as a result of 
close association, as a tie, hood; $(\mathrm{G})$ as that which nods; $(\mathrm{H})$ as that which gurgles; (K) unclassified.

40A1. MLG. bèr-swel Eberhals: bēr boar+swel Geschwulst.

40A2. EFris. pukkel Buckel; Nacken, NHG. buckel.

40A3. Swiss bläst das Blasen; etwas Aufgeblasenes; dicker Hals, MHG. blāst das Blasen; Blähung. Cf. 6E2, 85A7, $101 \mathrm{~A} 2$.

40A4. Swiss guge Nacken; Hals. Cf. 81B12.

40A5. Bav. hitzel (hützel) Genick, Nacken, Sax. huzel missratenes Obst; verschrumpfte Backware; im Wachsen zurückgebliebenes Geschöpf. Cf. 81B15.

40A6. Neumärk., Wald. huke Nacken. Cf. 89A7.

40A7. EDial. chump inferior end of neck of veal, etc. Cf. $2 \mathrm{~A} 20$.

40A8. EDial. knot nape of the neck. Cf. 2A29.

40A9. EDial. crease ridgetile of a roof; crest of a horse's neck, withers; verb fold up, NE. fold, ridge, wrinkle.

40A10. Lothr. kadutsch Genick. Cf. 5A20.

40A11. NE. scuff, scuft nape of the neck, NFris. skuft withers, EFris. schoft, MLG. schuft idem. Cf. 45A4.

40A12. NE. cuff nape of the neck. From 40A11.

40A13. EDial. scruff nape of the neck; skin or hair at the back of the head, scruft idem: from $s c(u f f)+(s c)$ ruff scurf (NED.). Cf. $40 \mathrm{~A} 11$.

40A14. EDial. scut nape of the neck. Cf. 92B7.

40B1. Lothr. akkaul, Lux. äkaul Nacken: ak neck+kaul, MHG. kūle Grube. Compare Lux. kratz-kaul, läuse-kaul Nacken.

40B2. Lux., Lothr. greiwel (gräbel) Nackengrube, Lothr. grub Grube; Nackengrube, Genick, OHG. gruoba Grube.

40B3. Als. gragel Hals: from kra(gen) + kehle. Wood, JEGPh., $\mathrm{XI}, 310$.

40B4. Lothr. hilsch Hülse; Nackenhöhle, Als. hülsche, hülse idem.

40B5. EDial. sheugh ditch, furrow, trench; hollow of the neck, NE. furrow, trench, etc.

40B6. EDial. scoop scoop; neck and breast of mutton cut together as one joint; narrow gully, etc. Cf. 40B7.

40B7. EDial. scovin neck and breast of a lamb, brisket, skuffin forequarter of a lamb after the shoulder has been taken off, scubbin 
idem, scovel, scovin mop used for cleaning out a baker's oven, scubbin idem. Cf. Fr. escouvelle broom. Cf. 40B6.

40C1. Oberh. wurzel Wurzel; Nacken, NHG. kopfwurzel idem.

40D1. Swab. gräte Hals; hoher Bergrücken. Compare Arnl. 109:01.

40E1. Thur. stich Kehlstück geschlachteter Tiere, NHG. stechen. 40F1. Prus. binde necktie; neck.

40F2. Lothr. kartusen, NHG. cartause Genick, Wald. kardusel, Bav. kartuss Hals. Cf. 5E10.

40G1. EDial. nod, noddle, niddle nape of the neck. Cf. 2 L3.

40H1. Swab. krigel Hals, Nacken, kriglen geräuschvoll räuspern, gurgelnd atmen.

40K1. NE. nape back upper part of the neck.

40K2. EDial. yop nape of the neck.

\section{0a. Withers}

The withers are described as that which resists.

40a. NE. withers ridge between the shoulder blades of a horse, NHG. widerrist idem, OE. wiðer against. Cf. $41 \mathrm{~B} 3$.

\section{Throat}

The throat is described (A) as an opening, channel, pipe; (B) as a swelling, lump; (C) as that which swallows; (D) as a noisemaker; (E) as a lamp (when burning with thirst).

41A1. Lothr. piff Pfeife; Kehle, NE. pipe.

41A2. Bav. flosse Kehle, floss gutter.

41A3. DuDial., EFris. halsgat throat, MDu. kelegat, Du. keelgat idem, gat hole.

41A4. EDial. hozle throat, windpipe. Cf. hoozle socket; eye of an axe; band of paper around a number of papers.

41A5. NE. cannel channel; pipe; throat, Lat. canalis channel (CD.).

41A6. Swiss chrachen Schlucht; Rachen, chrachen krachen; bersten.

41A7. Westf. trechter Trichter; Hals.

41B1. MDu. berle throat; ton; wine-cask.

41B2. EDial. chuck under part of the face; throat, pl. cheeks; lips of a dog, OE. cēake cheek. Compare Arnl. 35:07. 
41B3. EDial. witters throat (to be at a person's witters). Probably for withers. Cf. $40 a$.

41C1. Fris. swolge pharynx, Flem. swelg idem, swolgje swallow, Bav. schwalg Schlund, OHG. swelgan swallow.

41D1. Tirol. gīngger Hals, ginggitzen gicksen, gingger Gickser.

41D2. DuDial. goeber throat, goeberen make a humming sound; gush, goeber plaything made of a board and making a humming sound.

41D3. Westf. kijack Hals, Schnabel; Gans; Luftröhre der Gans, kijacken schnattern. Cf. 9L3.

41D4. DuDial. roeper throat, roepen rufen.

41E1. Prus. lampe Lampe; Kehle. Einen auf die Lampe giessen $=$ take a drink.

\section{LARYNX}

The larynx is described (A) as a lump, round object, swelling; (B) as to the glottis, as a hole; (C) as to the epiglottis as a flap.

42A1. NHG. adamsapfel, NE. adam's apple larynx.

42A2. Swab., Als. adamsbutzen larynx, butzen Klumpen.

42A3. Swiss bätzi Abfalle; Kerngehäuse; Adamsapfel. Cf. $90 \mathrm{~A} 4$.

42A4. Bav. göderlein larynx. Cf. $44 \mathrm{C} 1$.

42A5. Swiss gügi larynx. Cf. 81B12.

42A6. NHG. kehlkopf larynx.

42A7. Du. strottenhoofd larynx: strot throat+hoofd head.

42A8. MDu. cnoppe, Swab. knopf knot, knob; larynx, NHG. knopf.

42B1. Du. ademgat glottis. Cf. 10A2. NHG. atemloch idem.

42B2. Lux. vadronserlächelchen glottis (vaterunser-löchelchen) (Lux.).

42C1. Du. keellel epiglottis: keel throat+lel flap.

42C2. EDial. clack, -er epiglottis. Cf. 22E2.

\section{WINDPIPE}

The windpipe is described (A) as a pipe, tube; (B) as that which is throttled.

43A1. Swiss halsader, NHG. atemröhre, luftröhre, Swiss röhre, Du. luchtader, luchtpijp, NE. pipe, windpipe windpipe.

43B1. NE. thropple windpipe, verb throttle, strangle. 


\section{Gullet}

The gullet is described (A) as a gut, swallower; (B) as a stringlike object; (C) as a lump, swelling; (D) as a noisemaker.

44A1. DuDial. keeldarm gullet.

44A2. Du. slokdarm gullet, slokken schlucken.

44A3. Lux. schlek Schlund, schlecken schlucken.

44B1. Flem. fas gullet, NHG. faser.

44C1. Bav. goder Gurgel, Schlund; fleischige Haut unter dem Kinn; äussere Gegend vorn am Hals, Tirol. gouder fleischige Haut unter dem Kinn. Cf. 42A4, 14A10.

44C2. Bav. kreb Schlund. Cf. 83A2.

44C3. Sax. kuttel Gurgel; Eingeweide; sehniges, häutiges Fleisch; Pferdedünger. Compare Arnl. 135:32.

44C4. Swiss chrosse Gurgel, NHG. krossel Knorpel.

44C5. Sax. parabel Gurgel. Cf. 2 A7.

44C6. EDial. wame gullet; and as OE. wamb belly, stomach, womb. Compare Arnl. 103:12.

44D1. EDial. kecker gullet; windpipe; uvula, kecken make a sound between a choke and a cough.

44D2. Kärnt. kör'l Gurgel, Swab. kurren knurren, girren.

\section{SHOULDER}

The shoulder is described (A) as a lump or bump; (B) as something split off; (C) unclassified.

45A1. Lothr. bloiwel Waschbläuel; Schulterblatt des Schweines, MHG. bliuwel Bläuel, Stampfmühle.

45A1a. Du. bout shoulder, leg; bolt, NE. bolt.

45A2. Steir. lämmerbügel Fuss sammt Schulter eines Lammes.

45A3. Swab. krätze hohe Schulter eines Buckeligen. Cf. $89 \mathrm{~A} 11$.

45A4. EFris. schoft Schulter; Schulterblatt; Vorderbug beim Rind; Widerrist beim Pferd, Du. Schulter, Schenkel, NFris. skuft Widerrist, Rücken, Kamm des Halses, Westf. schuft Schulter, MLG. Widerrist des Pferdes. Cf. 40A11.

45A5. Swiss strauwsack Strohsack; Teil des Buges eines Ochsen. Cf. $1 \mathrm{D} 1$. 
45B1. EDial. spald shoulder; limb, joint, verb partially separate the shoulder; dislocate a joint.

45C1. DuDial. lo shoulder; space between shoulder and body. Cf. $48 \mathrm{C} 1$.

\section{Shoulder Blade}

The shoulder blade is described as something flat, a blade.

46A1. Prus. lapatte Schulterblatt, Schulterstück, Lit. lapatka Schulterblatt, dimin, of lopetà Schaufel (Frisch.).

46A2. Swiss laff Schulterblatt; Ruderblatt, Swab. laffe idem, Grube unter den falschen Rippen. Cf. 90E7.

46A3. EFris. spade-blad spade blade; shoulder blade.

46A4. EDial. spade-bone shoulder blade.

46A5. DuDial. schuiver shoulder blade, schuiven shove.

46A6. NHG. schulterblatt, NE. shoulder-blade, blade-bone.

\section{ARM}

The arm is described (A) in terms of the corresponding members of various animals; (B) as a knob (arm stump).

47A1. EFris. finne fin; arm. Cf. 50F1.

47A2. MDu. vlerc hand. Cf. $61 \mathrm{~A} 7$.

47A3. Lux. flīcht arm. Cf. 61A10.

47A4. EDial. fipper arm. Cf. 50F2.

47A5. EDial. wing arm.

47A6. Als. fienke Arm. Cf. 61A4.

47A7. EFris. flunk arm. Cf. $61 \mathrm{~A} 5$.

47B1. DuDial. knuist stump of an arm or hand, Du. knoest knob, knot.

\section{Elbow}

The elbow is described (A) as a point; (B) as a touchy thing; (C) as that which shuts.

48A1. Fris. earmtakke elbow, takke branch of a horn, etc., NHG zacke.

48B1. NE. funny-bone place at the elbow where the ulnar nerve passes by the internal condyle of the humerus. 
48B2. Cologne verwentsknöchelche funny bone, verwenne verwöhnen.

48B3. Cologne vexeerknöchelche funny bone, NHG. vexieren.

48C1. DuDial. luik bend of the elbow. Cf. $73 \mathrm{~B} 3$.

\section{WRIST}

The wrist is described (A) as the back part of the hand; (B) as a root; (C) as the pulse.

49A1. Du. achterhand wrist.

49B1. NHG. handwurzel wrist, Du. handwortel idem.

49C1. Du. pols pulse; wrist.

\section{HAND, Fist}

The hand is described (A) as a lump, knob, and the like; (B) as that which grasps, snatches, digs, feels, beats, etc.; (C) as something loose, flabby; (D) as a fork; (E) by the numeral five; (F) as the corresponding limb of an animal; (G) as Samson; (H) as a lefthanded person; (J) unexplained.

50A1. Swiss bratschen Werkzeug zum Schlagen; Schorf von Wunden; harter, festgewordener, breitgedrückter Gegenstand; plumpe Hand, Sax. braatsche dickes Weib; Hand, Swab. bratsche etwas Dickes, Plumpes; grosse Hände, Füsse, Sax. pratze Hand, Steir. bratzel Händchen. Cf. 7H3, 74B3, 85A42.

50A2. Swab. pfat Tatze; Hand, NE. paddy hand, pad bundle of straw, etc.; cushion-like part of the body. Cf. 52A2.

50A3. Swab. pflatsch grosse plumpe Hand; unbeholfener Mensch, pflatschig unförmlich fett, pflotze grobe, unreine Hand, Bav. pfloten Hand, Swiss pflütz Kot vom Rindvieh; fettes Weib.

50A4. Du. pol hand; stump. Cf. $2 \mathrm{~A} 8$.

50A5. EDial. daddle hand, daddes hands, dad lump.

50A6. Cologne tootsch Fackel; Hand, Fr. torche torch; bunch of twisted straw, etc.

50A7. Swab., Sax. talpe hand. Cf. 52A4.

50A8. EDial. spud potato; short stumpy thing; hand, spuddy stumpy.

50A9. Lux. hämmelsbichelchen grosse Hand. In NE. a large hand is compared to a ham. 
50A10. Swiss chloben, Lothr. klowen Kloben; Hand, MHG. klobe Kloben; Bündel, Büschel. Cf. 9A9, 74B5.

50A11. Lothr. klutte plumpe Hand, Als. kluttere dicke, plumpe Frau.

50A12. Cologne knävvele grobe Hände, NHG. knobel Knöchel am Finger, Swiss chnubel knolliger, rundlicher, derber Körper; grosses Stück, MHG. knübel knuckle.

50A13. Swiss chnode Hand. Cf. 149A4.

50A14. Du. knuist, Fris. knüst fist, hand; short thick man, Swiss gechnüst Gedränge, MLG. knüst knob, knot.

50A15. Cologne knuppe Hände, Pfoten, Swiss chnuppel Beule; Knorren; Knüttel; Knoten.

50A16. Swiss chnüttel Hand; Knüttel, Cologne knute Hände, MHG. knüttel knotiger Stock, etc. Cf. 53B4.

50A17. EDial. maeg large clumsy hand, verb handle, finger. Perhaps from Gael. mag paw (Jam.).

50A18. Swab. nockel Faust, Bav. nocken Hügelchen, Fels aus dem Wasser emporstehend, Swiss noggel knotiger Mensch.

50B1. Swab. faukele geschlossene Hand, Swiss pfüken stehlen.

50B2. Swiss griffing Hand, NHG. griff Klaue der Raubvögel, greifen.

50B3. Tirol. grampe Haue; Hand, MHG. krampe Spitzhaue.

50B4. Egerl. graubm Hand, Swiss graupen herumtasten, -krabbeln.

50B5. NE. clutch hand, EDial. cleuk hand, claw, paw; clutch, grasp, verb seize, grip; scratch with claws.

50B6. Swab. kralling Hand, krallen kratzen.'

50B7. Sax. krapeln Händchen; Vogelfüsse, Swiss chrapen herumtasten, -krabbeln; kratzen. Cf. 53E1, 60A7.

50B8. Swiss chrōpen die Finger; Hand. Cf. 50B7.

50B9. EDial. mauls, maulers hands, fingers, verb finger; handle roughly.

50B10. NHG. patsche Werkzeug zum Schlagen; (patschende) Hand oder Fuss, patsch schallender Schlag; Klatscherei.

50B11. Prus. prïtze unförmliche Hand, pritze leichtes gespaltenes Brett zum Schlagen; Schlegel. Cf. 92A2.

50C1. Swiss lāpen Finger, Hand, lāpen mit den Fingern plump, ungeschickt greifen; trendeln, lape schwach. 
50D1. Du. adamsvork hand, fingers.

50D2. Kärnt. gab'l Gabel; Hand.

50E1. NE. fives hand, fist.

50E2. Sax. fünfingrige Hand.

50E3. Sax. fünfzinkige Hand.

50F1. EFris. finne fin; hand; arm. Cf. 47A1; Arnl. 73:06.

50F2. NE. flipper hand, etc., flip toss, fillip. Cf. 47A4, 64A2.

50F3. NHG. flosse swimmer; hand, MHG. vlosse Flosse. Compare Arnl. 73:01.

50F4. DuDial. fleek (fneek) hand; claw, fleken scratch, Flem. vleek wing; hand. Cf. 61A8, 60E1.

50F5. Bav. klaber, klaper Hand, Swiss chläpen Klaue; Finger; Hand, MHG. klaber claw. Compare Arnl. 71:01.

50F6. DuDial. kladden hands, MHG. klate claw. Compare Arnl. 71:06.

50F7. NHG. klaue, DuDial. klauw claw; hand. Compare Arnl. 71:03.

50F8. DuDial. klaviren claws; hands; fingers: blend of $k l a(u w)+(c l a) v i e r$ hook, as is shown by the secondary form klauwier, MDu. clauwier, clavier hook (Franck).

50F9. Prus. klarre Hand. For kralle?

50F10. Sax. pranke paw; hand, MLat. branca claw.

50F11. NHG. pratze, bratze Tatze; Hand, Lat. braccium arm; claw; branch.

50F12. NHG. pfote Hand; Fuss, Flem. puutje, pootje hand, MLG. pote paw.

50F13. Swab. tape, Thur. tope, Als. tapen Pfote; Hand; Finger, NHG. tappe breiter, weicher Tierfuss, Rap. tape Fuss der Gans.

50G1. EDial. samson fist.

50H1. EDial. gallack left hand, gallit left-handed person, adj. left-handed.

50J. NE. goll; Sax. klamotten; EDial. lift-hause hand.

\section{Palm}

The palm is described (A) as something flat; (B) as a hollow; (C) as the space between the outstretched fingers; (D) as to the ball of the hand as a round object, lump. 
51A1. Hess. blacke flache Hand, blacken Stück; Flecken; Lappen; Flicken, MHG. blacke Fleck, Gegend; Flicklappen.

51A2. Swiss platti flache Hand, NHG. platt.

51A3. ME. dalle hand, NE. doll palm of the hand, dalle flat slab used for flooring, Fr. same (NED.).

51A4. Steir. ten palm, NHG. tenne threshing floor.

51A5. NHG. handteller palm.

51A6. Swab. handsohle palm, from fusssohle (F).

51B1. EFris. gapse, gepse gaffende, offene Hand; die aus beiden zusammengelegten offenen Händen gebildete schüsselartige hohle Vertiefung, NHG. gepse, gapse eine doppelte hohle Hand voll, gaffen palms, MLG. gepse Höhlung der beiden zusammengelegten Hände, NHG. gaffen, NE. gape.

51C1. NE span hand with fingers outspread, verb stretch.

51C2. EDial. yarkin space between forefinger and thumb; hollow of the foot; grasp of the hand, yark jerk, wrench; stretch to the fullest extent.

51D1. NE. ball, NHG. ballen, Du. bal ball of the hand or foot. Antwerp dial. transfers bal to hollow of hand or foot.

51D2. Swiss ber ball of hand or finger. Cf. 9A1.

51D3. EDial. bumble ball of hand or foot. Cf. 74B2.

51D4. NHG. maus, Du. muis ball of the thumb, MHG. müs muscle, biceps. Cf. 127A26.

51D5. Du. venusberg ball of the hand. From mons veneris.

\section{PAW}

The paw is described (A) as a lump, pad, etc.; (B) as a shovel; (C) as a crown; (D) as a hand; (E) as that which patters, trots; (F) unclassified.

52A1. Cologne knuppe Pfoten. Cf. 50A15.

52A2. Swab. pfate Tatze. Cf. 50A2.

52A3. Swab. pflate Pfote; Kralle; Krebsschere. From pfl(atsch) $+(p f)$ ate. $\quad$ Cf. 50A2, 3.

52A4. Bav. talpen, Obersächs. talpe Pfote, Swab. talpe Pfote; ungeschickter Mensch, talpen stampfen, tappen. Cf. 50A7.

52B1. Als. schaufel (schufle) Maulwurfstatze.

52C1. Swiss chron Pfote der Hunde, Katzen. Cf. 79D1. 
52D1. Swiss hand vordere Tatze des Bären.

52E1. Steir. trappel Pfote, Fuss, NHG. trappeln trot, etc.

52F1. NHG. pfote, Du. poot, MDu., MLG. pote paw.

\section{Finger}

The finger is described (A) as that which catches, grasps, scratches; (B) as a lump, knot, etc.; (C) as a horn; (D) as a piano key; (E) as a hook; (F) as a claw.

53A1. EDial. fang eyetooth; claw; fin; finger. Compare Arnl. 32:13.

53A2. Swab. griffling finger. Cf. 50B2.

53A3. EDial. clamper claws; fangs; pincers; fingers, clamp a vise, mechanical cramp.

53B1. Swiss chlote Finger; Klecks, chlot Klecks; Knollen von Kot an Tieren, NHG. kloder Klumpen Schleim. Cf. 126A13.

53B2. Sax. klunker Troddel; überhaupt Gehänge; Klumpen; Finger, NHG, Kotklümpchen; Samenknollen; Fetzen. Cf. 92F3.

53B3. Swiss chnode Finger. Cf. 149A4.

53B4. Swiss chnüttel Finger. Cf. 50A16.

53C1. Swiss horn ausgestreckter Finger.

53D1. Sax. klaveten Finger, klaven piano keys, Fr. clavier keyboard. Cf. 19E1, 127P1.

53E1. Swiss chräpel hakiger Schriftzug; gekrümmter Finger, MHG. chrape Haken, Klammer. Cf. 50B7, 60A7.

53F1. Swiss chlāpe, Bav. klaber Finger, MHG. claber claw. Compare Arnl. 71:01.

53F2. Bav. klauber, kloper, Lothr. klope Finger, MHG. klouber claw. Compare Arnl. 71:05.

53F3. Prus. pazuren Finger, Polish pazur claw; finger nail (Frisch.).

\section{Thumb}

The thumb is described as a bug-crusher.

54A1. DuDial. tuutjeknipper thumb: tuutje bug +knipper crusher.

\section{Forefinger}

The forefinger is described (A) as a fork; (B) as a potlicker.

55A1. Steir. adamsgabel Zeigefinger und Daumen der rechten Hand. 
55B1. ME. lik-pot forefinger of the right hand, lit. lick-pot.

$55 \mathrm{C} 1$. EDial. currag forefinger. Unexplained.

\section{Middle Finger}

The middle finger is described (A) as something in the middle; (B) as the long one; (C) as grandmother.

56A1. MLG. middelink Mittelfinger.

56B1. NE. tall-man middle finger.

56B2. Westf. langman, lange-martin, Altmärk. langmeier Mittelfinger.

56B3. Swiss der grosse peter der grosse Finger.

56C1. Fris. äldmoer grandmother; middle finger.

\section{Little Finger}

The little finger is described (A) as something small; (B) as a tube; (C) as a doctor; (D) unclassified.

58A1. Swiss pfucher Knirps; kleiner Knabe; kleiner dicker Mensch; der kleine Finger, Swab. pfuche Geschwur; kleine Wunde oder Narbe.

58A2. EFris. pinke, pink der kleine Finger; Endchen Wurst; Darm, Wanst, Du. pink der kleine Finger; einjähriges Kalb, EDial. pink to make small, contract, esp. of eyes, pinker small. Cf. 123J5.

58A3. Cologne stubbenditzche der kleine Finger, Fr. tout petit (Hoenig).

58B1. EDial. crannie, creenie, curnie the little finger, cran a bent tube used to draw off liquor, tap, LG. kraan Fasszapfen.

58C1. MLG. arstedier little finger (used as a probe), arstedien to doctor.

58D1. EDial. okabell fourth finger.

59. NAIL

The finger nail is described (A) as that which scratches; (B) as a scoop; (C) as a rounding top; (D) as blossoms (white spots).

59A1. Swiss chräubel Fingernagel; Akt des chräublens mit Nägeln kratzen, chrüblen aufkratzen; am Brot klauben.

59B1. Cologne schöpp Schüppe; langer Fingernagel. Cf. 7H13.

59C1. Sax. kuppe Spitze des Fingernagels, NHG. kuppe.

59D1. Swiss bluest weisse Flecken der Fingernägel. Cf. 35A1. 
60. Claw

The claw is described (A) as that which catches, scratches; (B) as a hook; (C) as a point; (D) as something undivided; (E) as some other member; (F) as a food holder (the hind talon).

60A1. Swab. fang pl. Füsse der Raubvögel. Cf. 53A1.

60A2. NE. clive claw, talon, OE. clifian cleave, climb (NED.). Or Cf. $60 \mathrm{~A} 3$.

60A3. Du. kluif claw; fist, EFris. klufe, MDu. cluve, cluuf claw, MLG. kluve gespaltenes Holz als Fessel, kluven fesseln; spalten; klauben.

60A4. Swiss cluppen Klaue, clüperen verschiedene Geräte zum Kneipen; einwärts, gegeneinander gebogene Hörner eines Rinds; Krebsschere.

60A5. NE. clutch paw; talon, claw. Cf. 50B5.

60A6. EFris. knīper Krebsscheere, NHG. kneifen.

60A7. Lothr. krabele, Swiss chräbel Kralle, EFris. krabber Kratzer; Nagel, Kralle, NHG. krabbeln. Cf. 50B7.

60A8. NHG., EFris. kralle claw. From *kradlo-: kratzen (ZfdA., XLII, 56).

60B1. NE. cramp claw; paw; piece of iron bent at the ends, OHG. chrampha cramp, hook, clasp.

60B2. Swiss höggli Krallen einer Katze, OHG. hăgo, NHG. haken.

60C1. Prus. zanke Kralle; Zacke, MHG. zanke Zacke; Spitze.

60D1. EDial. single talon of a hawk. Cf. $92 \mathrm{~N} 1$.

60E1. DuDial. fleek claw. Cf. 50F4.

60E2. Swiss bratze Klaue. Cf. 50F11.

60F1. Du. aas-klauw hind claw of hawks.

\section{WING}

The wing is described (A) as that which flaps, flutters; (B) as a garment; (C) as down.

61A1. Als. flättig, flittig Flügel, Sax., Oberh. flittich Flügel; herabhängender, flatternder Gegenstand: $f a(t t e r n)+(f i) t t i c h$. Cf. $61 \mathrm{~A} 2$.

61A1a. Kärnt. fitte, Bav. fitschen Flügel, fitschen flattern. 
61A2. Swiss flättach Flügel: flatt(ern)+MHG. (vet)ach (Schw.). 61A2a. Kärnt. flatsche wing, Bav. flotsche idem, large feather, NHG. flatschen breites Stück; Fetzen. Cf. 7G3.

61A3. EFris. flüte swimmer; wing; Fetzen, Lappen, NHG. flosse.

61A4. Als. fienke Flügel; überh. was nicht fest mit dem Rumpf verwachsen ist, Swiss fliengge breites Stück von fester oder klebriger Mass. Cf. 47A6.

61A5. EFris. flunke Flügel; Rockzipfel; Aermel; Kragen, Flem. flonk dirty cap, hat. Cf. 47A7.

61A6. Lux. flilek Flügel eines Vogels: contraction of *flügelek, NHG. flügel. Compare Arnl. 72:02.

61A7. Du. vlerk wing, MDu. vlederic, vleric, vlerc, Westf. flerk Flederwisch: from Germ. *fleparo wing, formed under the influence of fiegen from Germ. *feparo (Franck).

61A8. Flem. vleek wing: probably a contraction of MDu. vleric. Cf. 61A7.

61A9. West. flürk Flügel: from $f l u ̈(g e l)+(f l e) r k(61 \mathrm{~A} 7), \mathrm{NE}$. firk flirt.

61A10. Lux. flīcht Flügel, Prus. flocht idem, Flügelartiges, NHG. fiegen.

61A11. NFris. jük Flügel, Swab. jucken hüpfen, springen.

61B1. Swiss hemd wie nhd.; die unter den äussern Flügeln liegenden Hinterflügel der Käfer.

61B2. Swiss rock rote Flügeldecken des Marienkäferchens.

61C1. Du. wiek wing; lint, MDu. wieke, DuDial. wuuk idem, NHG wieche Docht, Scharpie.

\section{Feather}

Feathers are described (A) as a covering; (B) as down; (C) as a rudder; (D) as a sickle (tail feather); (E) as a winnow (wing feather); (F) as a point, stub.

62A1. NE. covert protection, shelter; feathers covering the bases of the large feathers of wing and tail of a bird.

62A2. Swiss munter Uniform; Gefieder eines Vogels; Schale von Kartoffeln, Swab. montur Kleidung, Fr. monture Ausrüstung. 
62B1. Prus. posen Federn, pose Federkiel; Knallbändchen an der Peitsche, Thur. Flaumfeder, EFris. Feder, Federkiel. Wg. connects with NHG. buse feine kurze wollige Härchen wie Flaum. Cf. $127 \mathrm{G} 4$.

62C1. NE. rudder bird's tail feather, OE. rōpor oar, paddle.

62D1. Swiss sichle Sichel; Schwanzfeder des Hahns. Cf. 69C6.

62E1. NHG. wannen large wing feathers, Lat. vannus winnow. Cf. $68 \mathrm{C} 1$.

62F1. EFris. pile, pil pin feathers; downy beard, NFris. pill pin feathers, OE. pill, NHG. pfeil. Compare NE. pin-feather.

62F2. EDial. pug penfeather; quill left in a plucked fowl; anything short, thick, puggy short and stout.

62F3. EDial. stub stump; pin feather, OE. styb stump, stub, Als. stupfel pin feather. Cf. 19B7.

63. Fin

The fin is described as a wing, feather.

63A1. Swiss fischfecken Flosse, fecke, feckte, NHG. fittich.

63A2. Swab. feder Fischflosse. Cf. 92H1.

\section{FLIPPER}

The flipper is described (A) as a fan, flipper; (B) as a lump.

64A1. NE. fan fluke of a whale.

64A2. NE. flipper limb used for swimming. Cf. 50F2.

64B1. EDial. maeg flipper of a seal. Cf. 50A17.

\section{5. Сrotch}

The crotch is described as a fork.

65A1. NE. crotch fork; pole having a forked top; fork of the human body where the legs leave the trunk.

65A2. EDial. fork bifurcation of anything; of the human body.

65A3. Swiss griggele Gabelung; die der Schenkel des menschl. Leibes; Winkel zw. den Fingern; gabelförmiges Gerät.

65A4. Swiss grittele human crotch, MHG. griten spreitzen.

65A5. Swiss grippele meaning as 65A4 and derived from it.

65A6. EDial. kleevins fork of the body; cleaving, NE. cleave. 
65A7. DuDial. mik forked stick; crotch, MDu. micke forked instrument.

65A8. DuDial. spriet fork; crotch, MDu. pike; fork.

65A9. EDial. twist junction of the inside thighs of cattle, NE. human crotch, ME. branch.

\section{Groin}

The groin is described (A) as a bend; (B) as a fork; (C) as a border; (D) as something smooth; (E) as the part grasped.

66A1. EDial. clitch groin; fork of leg or arm; part between the legs of a pair of trousers, NE. to crook, bend, ME. clicchen to bend, incurve (Wr.).

66B1. NE. grain tine, prong; fork of a tree or stick; the groin, Swed. gren branch; stride; fork. Compare Arnl. 74:03.

66C1. EFris. lēste Leiste, Biegung der Schamgegend, MDu. lyst diaphragm, Hess. lāste, Swiss līst Leiste; Weiche, Bauchwand; Zwerchfell, MHG. līste Saum, Borte, NHG. leiste.

66D1. Steir. schlichten groin, NHG. schlicht.

66E1. EDial. ketch groin, NE. catch. Cf. 90S2, 140D1.

\section{LIMB}

The human limb is described as a wing in Swab. flügel human limb.

\section{ThIGH}

The thigh is described (A) as a lump, rounding object, club, stump; (B) as flabby; (C) as a winnow; (D) as something hanging; (E) as breeches.

68A1. Westf. batsen Oberschenkel. Cf. 90A4.

68A2. Wald. bol, Westf. bollen Schenkel, MLG. hamelsbolle Hammelskeule. Cf. 26E1.

68A3. Oberh. dickbein thigh, dick from MHG. diech, OHG. dioh thigh, being confused with dick thick. Compare Arnl. 76:07.

68A4. Swiss cholben Hinterschenkel geschlachteter Tiere. Cf. $5 \mathrm{~A} 32$.

68A5. EFris. küle, MHG. keule club; thigh, MHG. kiule club. 68A6. NFris. lurreg thigh, Norw. lurk Knüppel, dicker Stock. 
68A7. EFris. nagelholt Oberschenkel, Lende, NHG. nagelholz zähes, aus geraden Adern bestehendes Eichenholz. Cf. 141A7.

68A8. Als. schlegel Holzkeule; grosser Schinken, NHG. hammerartiges Werkzeug; Hinterkeule eines geschlachteten Tieres.

68A9. EDial. shiver slice; splinter; thick end of a leg of pork, Germ. *skif split (NED.).

68A10. MLG. stūt Oberschenkel, Steiss, NHG. steiss der Hintere, stotz Baumstumpf; Keule, Schenkel, stotze stark abgestumpfter und so niedrig gebliebener Baum. Compare Arnl. 112:24.

68B1. Als. qualle Hinterkeule, nicht geräuchert; starkes, dickes Mädchen, NHG. qualle, Du. qual jellyfish.

68C1. NHG. wanne ham. Cf. 62E1.

68D1. NHG. hanke Hüfte; Schenkel des Pferdes, Tirol. henkel Schenkel, Stück geräucherten Fleisches, das über dem Herde hängt, MDu. hanke hip, thigh, henken aufhängen.

68E1. Swab. hose Hosen; Oberteil des Hinterbeines beim Pferd; die Befiederung der Beine bei Vögeln. Cf. 90K1.

68E2. EDial. gaskins, gascoigns gaiters; thighs of a horse, NE. gaskin kind of breech or hose.

\section{LEG}

The leg is described (A) as that which runs, taps, pecks, bends; (B) as a pin, peg, post, stick, stalk, stump; (C) as a bend, crook, angle; (D) as a swelling, lump; (E) as a fork; (G) as catchers (bowlegs); (H) as stockings; (J) as boarding-house bones.

69A1. NHG. lauf, lauft, Lux. lāf leg of wild animals. Cf. 74A4.

69A2. DuDial. pekkel knucklebone; leg, pekken to peck, pekkelen rapidly moving legs, Antw. pikkel leg.

69A3. Wald. schuoken Beine, NHG. schocke Schaukel.

69A4. DuDial. steubeltjes legs of small children, steuven run.

69A5. Sax. strampel Beine, Lothr. stramplen idem, Schenkel, Lux. strömpel kurzes, dickes Bein, NHG. strampeln struggle, fidget, kick.

69A6. Sax. tippelchen Fuss; Bein. Cf. 74A7.

69A7. NE. bender leg.

69B1. Sax. bornständer water cask; thick legs, stand Gefäss. 
69B1a. Sax. hölzer, NHG. spazierhölzer Beine.

69B2. Swiss chelle Bein; Schlagholz. Cf. 74B7a.

69B3. DuDial. kuierstok cane; $p l$. legs, kuieren walk.

69B3a. EDial. leomen legs, OE. leomu limb.

69B4. Swiss lü̈chse Stemmleiste am Wagen; Beine, leuchse Stutzholz für die Leitern am Leiterwagen.

69B5. EDial. peg. leg, foot. Cf. 19B4.

69B6. EDial. pestle shank or foreleg, NE. club-shaped instrument for pounding, OF. pestel pestle.

69B7. EDial. pin leg. Cf. 167E1.

69B8. EDial. post thick leg.

69B9. Sax. spazierklötzer Beine, klotz chunk of wood.

69B10. Wald. stalen Bein, MLG. stale Stolle, Bein oder Fuss (einer Kiste). Cf. 104A13.

69B11. Lothr. stolle Stütze aus Eisen oder Holz; Bein des Menschen, NHG. stollen prop, post. Cf. 69B10.

69B12. DuDial. stapel leg, stapeleeren stand firm, Du. stapel leg of a chair.

69B13. Als. stecken Stecken; dünne, magere Beine. Cf. 123B6.

69B14. EFris. stilte, stelter Stange, Stengel; Bein, Lende, Keule, stelte Stelze, NE. stilt.

69B15. EDial. stumps legs.

69B16. EFris. stutte Stutze; Bein, NHG. stütze.

69B17. EDial. tram beam, bar, shaft; wooden framework for supporting casks; limb, leg; one with long ungainly limbs, EFris. trame, trām beam of wood, rung of a ladder.

69B18. EDial. traneen kind of grass; straw for plaiting hats; long thin legs, Ir. traithnin, trathnan little stalk of grass (NED.).

69C1. EDial. bucker horse's hind leg; bent piece of wood on which slaughtered animals are hung, NE. buck to bend, buckle.

69C2. EDial. cambrel hock of any animal, NE. cambrel, gambrel, OF. gambereaulx crooked stick for hanging meat (NED.).

69C3. EDial. cantle corner, projection; leg of a lamb or any other young animal, OF. cantel corner; piece, bit. Cf. $32 \mathrm{C} 4$.

69C4. Kärnt. hackse Bein, magere oder krumme Beine, Thur., Sax. hachse Hinterbein, MHG. hahse Kniebiegung an den Hinterfüssen. Cf. 78A1, Arnl. 81:04. 
69C5. Swiss chrucke Krücke; Bein; Fuss, Bav. kruecken Krummbeine; Beine überhaupt, NHG. krücke.

69C6. Swiss sichle Sichel; krumme Beine. Cf. $62 \mathrm{D} 1$.

69D1. EDial. spung leg of a fowl, drumstick; purse, fob, pocket.

69D2. EDial. slench big piece or lump; hind leg of beef, slenching big piece or lump.

69E1. Swab. gabel Gabel; gespreizte Beine. Cf. 55A1.

69E2. Swiss geigle Doppelast an einem Baume; pl. die entblössten Schenkel, Beine beim Fallen in die Höhe gestreckt, geiglen üppig ranken und schwanken, in steter Bewegung sein.

69E3. Schles. gragel gabelförmiger $\mathrm{Z}_{w e i g}$, die gespreizten Beine, grageln breitbeinig, ungeschickt und schwerfällig gehen, die Beine in die Hö he recken.

69E4. Bav. graitel die gespreizten Beine, graiteln auseinander sperren, Lothr. graitsch grosser Schritt. Cf. 65A4.

69G1. DuDial. knijnetakkers bowlegs: knijn rabbit+takken catch.

69G2. DuDial. schapevangers bowlegs (sheep catchers).

69H1. EDial. moggan stocking; $p l$. legs, Gael. mogan stocking without foot (Wr.).

69H2. Steir. strumpf horse's white leg, as NE. stocking idem.

69J1. Sax. pensionsknochen Beine.

\section{Calf}

The calf is described (A) as something rounding; (B) as something soft; (C) unclassified.

70A1. EDial. ball calf of the leg; palm of the hand; sole of the foot. Cf. 51D1.

70A2. EDial. turning calf of the leg; space between calf and ankle.

70B1. NE. brawn fleshy part of the body, muscle, esp. the rounded muscles of the arm, leg, thumb; arm; calf of the leg; buttocks, OF. braon, braoun fleshy part, from OLG. brädo Braten.

70B2. MDu. cute, Du. kuit calf of the leg; ovaries; fish spawn, MLG. kut, kute das Weiche, Knochenlose im Tierkörper; Eingeweide; Rogen; Wade, EFris. küte, kü̈t Wade; Eier der Fische. Cf. 113B5. 
70B3. MDu. lire, liere calf of the leg, OE. lìra fleshy part of the body without fat or bones. Compare Arnl. 153:04.

70C1. EDial. tibby calf; calf of the leg.

\section{KNEE}

The knee is described as a knob, lump.

71A1. NFris. knöbbian Knie, Du. knobbel knob, knot.

71A2. EDial. knogs knees; shank-bones, Norw. knog bone, knuckle (Wr.). Compare Arnl. 69:08.

71A3. NHG. häspe Kniebug, Du. hespe leg of pork, ham (Kil.), MHG. haspe Garnwinde.

\section{KNEEPAN}

The kneepan is described as a pan; disk; cap; knob.

72A1. Fris. panne kneepan, NE. kneepan. Cf. 37C5.

72A2. EFris. smer-schīfe Kniescheiben-Gelenk: smer oily substance+schĩfe Scheibe.

72A3. NE. kneecap kneepan.

72A4. EDial. knap bump; knob, knot; knoll; kneecap; point of the elbow.

\section{Bend OF the KneE}

The bend of the knee is described (A) as a hollow; (B) as a pincher, shutter; (C) as the veined part.

73A1. EFris. kule, kül Vertiefung, Grube; Kniekehle, MHG. kule Grube. Cf. 40B1.

73B1. Fris. knibbel knee, Du. knibbels bent knees, EFris. knibbeln gnaw; pinch. Cf. 123E5.

73B2. DuDial. nepe bend of the knee or elbow, neep that which pinches, squeezes; twitch, Du. neep pinch, twinge.

73B3. DuDial. luik, loke in bend of elbow or knee, Du. luik hatch; shutter, MDu. luken shut, clench. Cf. $48 \mathrm{C} 1$.

73C1. Swab. geäder Gesammtheit der Adern; Beugseite der Gelenke; Handgelenk, Fussgelenk; Kniekehle, gang-ader Kniekehle.

\section{Foot}

The foot is described (A) as that which dances, goes, walks, runs; splashes; shuffles; (B) as a knob, lump, chunk, swelling; 
(C) as a stand; (D) as a stalk; (E) as a dangle; (F) as a horse;

(G) as a hoof, paw.

74A1. Westf. danss Tanz; Fuss des Hasen, Fr. danse.

74A2. Swiss gänger, Swab. gängling Fuss, EDial. gangers feet of a bullock, NHG. gang.

74A3. NE. goer foot: go.

74A3a. Prus. klisch Fuss, Bav. kleschen klingen, klatschen.

74A4. Swiss, Swab. lauf Fuss, DuDial. schoaploop feet of a slaughtered sheep, NHG. lauf. Cf. 69A1.

74A5. Sax. latsch grosser Fuss, Lothr. latschen idem, Pantoffeln, Als. latschi Fuss; läppischer, träger Mensch, NHG. latschen shuffle, slouch.

74A6. EDial. scrapers heels, feet: scrape.

74A7. Sax. tippelchen Fuss, tippeln tüpfeln, in kleinen Schritten gehen, NE. tip strike smartly but lightly; tap noiselessly; step lightly, walk mincingly. Cf. $69 \mathrm{~A} 6$.

74A8. Steir. tramper plumper Fuss, NE. tramp.

74A9. Steir. trappe plumper, grosser Fuss; Tölpel, trappel Fussspur; Teil des Fusses, der den Boden berührt; Pfote; Fuss, Fris. traper foot; shoe, Du. trap kick, MDu., MLG. trappen tread, trample.

74A10. Sax. tratscher Fuss, tratsche grosser, ungeschlachter Fuss, tratschen schwerfällig, derb, plump und hörbar auftreten.

74A11. Swab. trittling, Steir. treter Fuss, NHG. treten.

74A12. NE. walker foot; leg: walk.

74B1. Fris. bonken feet. Cf. 167B1.

74B2. EDial. bumble bundle, thick, clumsy package; elubfoot, verb bungle, stumble. Cf. 51D3.

74B3. Swab. bratsch grosse Füsse. Cf. 50A1.

74B4. EDial. fottys children's feet, fottie one whose stockings, trousers, boots, etc., are too wide; plump, short-legged person or animal. Cf. 5A12.

74B5. Lothr. glowen plumper Fuss. Cf. 50A10.

74B6. EDial. hommock heap, large piece or slice; awkward, clumsy person; large awkward foot or leg, hummock hillock.

74B7. DuDial. kaakje foot, Du. cake from NE. cake.

74B7a. Swiss chelle Löffel; Schlagholz; Fuss, MHG. kelle Schopfgefäss; Hütte; Tümpel. Cf. 69B2, 123B8. 
74B8. EDial. clump lump, mass; heavy, inactive person; heavy stick; awkward feet, NHG. klumpen.

74B9. EFris. klunt Klumpen, Kloss; Klotz; grober, lautauftretender Fuss, Du. klont lump, clot; knob.

74B10. Swiss chnochen Fuss, MHG. knoche Knochen; Astknorren; Fruchtbolle.

74B11. Swiss chnode Fuss. Cf. 149A4.

74B12. Prus. knoss grosser Fuss, Swiss chnosi wohlbeleibter Mensch; kleiner pausbackiger, draller Knabe, Swab. knosig fest, knollig.

74B13. Fris. lobbe foot. Cf. 15A3.

74B14. EDial. pad foot of an animal, NE. pad. Cf. 50A2.

74B15. EDial. pod, poddy foot, NE. poddy corpulent, pod seed vessel, etc. Cf. 85A40.

74B16. EFris. pikkel Füsschen, Du. foot of a table, bikkel small bone; marble, MHG. bickel Würfel; Knöchel.

74B17. Prus. quante grosser, ungeschickter Fuss, Cologne quant dickes, ungezogenes Kind, Du. lustiger Bruder, Bude, LG. buffoon.

74B18. Lothr. datsch breiter, plumper Fuss; Hand. Cf. 3A2.

74B19. Thur. tope Fuss. Cf. 50F13.

74C1. NHG. stand das Stehen; Fuss der Vögel, NHG. ständchen stilt.

74D1. Swab. dors Stengel, Strunk von Kohl, Salat samt dem markigen Innern und den Blattrippen; magerer Fuss; dürrer Mensch, MHG. torse Kohlstrunk.

74E1. Swab. bampferlein Füsse, Sax. pampeln hangend sich hin- und herbewegen, bammeln.

74F1. Cologne apostelpääd Füsse, pääd Pferd.

74G1. EDial. hoof foot.

74G2. DuDial. poot foot of animals and men, Fris. poat idem, DuDial. poetel foot of children. Cf. 52F1.

74G3. Pruss. pranke Tatze; Fuss. Cf. 50F10.

75. SoLE

The sole of the foot is described (A) as a flat surface; (B) as a frog.

75A1. Swab. britte grössere Fläche Feldes; etwas zum trocknen Ausgebreitetes; Fläche des menschlichen Fusses; Oberteil des 
Fusses, NHG. brett, OHG. breta palm of the hand, Bav. britten Rücken des Fusses. Compare Arnl. 57:01.

75B1. Fris. frosk frog; sensitive part of a horse's hoof.

75B2. NE. frog animal; elastic substance growing in the middle of the sole of a horse's hoof.

\section{INSTEP}

The instep is described (A) as that which is free, unobstructed; (B) as a swelling; (C) as a span.

76A1. DuDial. vrei, vrij instep, vrei open space, Du. vrije idem.

76B1. DüDial. krop, krap instep, NE. crop.

76C1. NHG spann Fussrücken, EFris. span idem. Cf. 51C1.

\section{ANKLe}

The ankle is described as a lump, knot.

77A1. MLG., Swab., Swiss bickel Knöchel; Würfel, MHG. Würfel.

77A2. Als. knode Fussknöchel; kurzer, dickér Kerl, Swiss chnode Knoten, Fussknöchel, Knöchel am Handgelenk, Bav. knoden Knöchel; Knollen. Cf. 149A4.

77A3. Du. knoesel ankle, Flem. idem; gooseberry, MDu. cnoesel ankle, cnoes cartilage, Swed. knast gnarl, knot.

77A4. Als. knolle Knöchel, Fussgelenk; Klümpchen; Grobian, Swiss Klumpen; kurzer, dicker Mensch.

77A5. Prus. knorre, knurre Knorren; hervorstehender Knochen, Enkel; Beule, Auswuchs, Knust, NHG. knorren.

\section{HEEL}

The heel is described (A) as a crook, bend, angle; (B) as a tendon; (C) unclassified.

78A1. Rogas. hes Ferse, Fuss, Prus. hesse Hinterfuss; Ferse des Menschen. Cf. 69C4.

78A2. DuDial. huk heel, hukken to bend in, Cologne huche Hacken, Fersen, huche hocken, Swed. huk nook, point.

78B1. Flem. vas, fas heels. Cf. 150D1.

78C1. Flem. fuk, fok heel. Cf. $9 \mathrm{H} 1$.

78C2. EFris. hide Ferse. 
79. Hoof

The hoof is described (A) as a knot, lump, stump; (B) as a flat object; (C) as a clapper; (D) as a crown; (E) as to the upper edge as a border; (F) as a cleft; $(G)$ as an arrow (middle of a horse's hoof).

79A1. NE. cloot one of the divisions of a cloven hoof; hoof, Bav. kleuzen spalten (Wr.), ON. klo or Ger. *klow(a) claw (NED.), Du. kloot lump, ball, NE. cleat.

79A2. MLG. kote, kute Huf, Klaue; Fessel beim Pferd; Knöchel; Würfel, NHG. köte unterstes Gelenk am Pferdefuss, OFris. kāte Knöchel. Compare Arnl. 110:09.

79A3. EDial. cowk cow's hoof, coke, cowk core or pith of anything; heart of horns; remnant of a stack of hay, NE. colk core of fruit, of a horn, heart of wood. Of uncertain origin (NED.).

79A4. Westf. mutte Klaue des Hornviehs; Sau, Swab. mutz Sau, mutze Tier mit gestutztem oder kurzem Schwanz, Als. mutz Huhn ohne Schwanz, Swiss mutz gestutzt, stumpf.

79B1. EDial. loof palm; hoof, ME. lōve palm. Compare Arnl. $57: 08$.

79C1. Swiss chlaffe Klapper, etwas Klapperndes; grosser Huf eines Rindes, der nicht beschnitten ist und klappert, MHG. klaffe Klapper.

79D1. Swab. kron oberer Rand des Pferdehufes, Krone. Cf. $52 \mathrm{C} 1,146 \mathrm{E} 1$.

79E1. Swab. breis Schlusssaum an Kleidern; oberer Saum des Pferdehufes, MHG. brīse, brīsem Einfassung, Einschnürung an Kleidungsstücken, brïsen schnüren.

79F1. MLG. snēt gespaltene Klaue, Hufe des Viehes, sniden schneiden, schlitzen, NHG schneiden.

79G1. Du. straal, hoornstraal arrow, middle of a horse's hoof, NHG. strahl idem, MHG. strale arrow. Cf. 148A5.

\section{ToE}

The toe is described (A) as a knob, lump; (B) as a point; (C) as a gold toe.

80A1. EDial. knobs toes. Cf. $2 \mathrm{~A} 27$.

80A2. EDial. megh big toe. Cf. 50A17. 
80B1. MDu. timp pointed outer end; point; toe, Du. pointed outer end.

80C1. MDu. gout-tee toe next to the little toe, corresponding to gout-vinger ringfinger.

\section{Breast, Udder, Teat}

The breast, udder, or teat is described (A) as a vessel, container; (B) as a lump, swelling, protuberance, etc.; (C) as something hanging, dangling; (CC) as something soft; (D) as that which is sucked, suckles; (DD) as something stroked; (E) as that which gives milk; (F) as a nourisher; (G) as a dripper; (H) as a mother; (J) by proper names; (K) as the seat of power; (L) as something good; (M) as a quarter; (N) as something by the udder (an extra teat); (P) unclassified.

81A1. NE. bag sack; udder. Cf. 104B3.

81A2. Als. fass Fass; Euter; Brust, OHG. faz Fass.

81A3. Swiss fläsche Flasche; Euter; Brust, Fris. flesse bottle, breast, MHG. vlasche Flasche.

81A4. EDial. vessel pail, etc.; udder.

81A5. Swiss gehalt Behälter; Zitze, MHG. Gefängnis, etc.

81B1. Bav. bamazln Zitzen. From ba(tzl) kleiner Batzen+ *mazln (Bav. matz nackte Schnecke; knorriger Holzblock, Klotz). Wood, JEGPh., XI, $300 \mathrm{f}$.

81B2. Steir. banazel, panatzel jungfräuliche Brust. From pan $(z l)$ dimin. of panzen breites Fass, bauchiges Geschirr; Bauch+(b)atzl. Cf. 81B1, with ref.

81B3. Cologne bosch Brust, Swiss Busch, Buschartiges, MHG. idem.

81B4. Swiss bruschi Brusttuch; Brust, Busen. Cf. 119A1.

81B5. Swiss büz Euter der Kuh. Cf. 5A10.

81B6. Schles. pitz Busen, LG. pitze Brustwarze, Prus. pize weibl. Brust, Sax. piez, pieze Euter, weibl. Brust, Cologne pieze, Thur. biez Brustwarze, NE. pit stone of a cherry, etc.

81B7. Du. pram breast, EFris. pram Brustwarze, Saugwarze, MLG. premese twitch, EFris. prame Klemmholz, Nasenkneipe. Cf. $85 \mathrm{~A} 43$.

81B8. Swiss puppe Brustwarze, Zitze, Habkern püppi Mutterbrust. Cf. 5A9. 
81B9. Swiss butti Brust; hölzernes Gefäss, woran die Kinder saugen. Cf. 85A11.

81B10. Oberh., Als. dutte, Bav. dutten weibl. Brust, Swab. dutt Brustwarze, Brust, Zitze, Euter, Als. düttel Brustwarze des Mannes, Brust, Zitze, Nösner tuttel Brust, Bav. dütze Euter, Hess. dutzen, dütz idem. Cf. 7H12, 114A1.

81B11. Swab. dülle Saugwarze, Mutterbrust, duller Blutgeschwur, Norw. dulla little round thing or person. Cf. Wood, Mod. Phil., XI, 334.

81B12. Swiss gūge Brüste; Blase, Geschwür, Swab. gügerlen hoch aber schlecht aufeinander haufen, Swiss güggi Anschwellung auf dem Gesicht oder Arm. Cf. 40A4, 42A5, 90A32, 123C15, 5A29.

81B13. Tirol. gundele Brust. Cf. 85A22.

81B14. Swab. holz Holz; Die hat Holz vorm Haus, an der Wand $=$ volle Brüste.

81B15. Sax. huzel Brüste; Hess. hutz weibl. Brust. Cf. 40A5, 127A21.

81B16. Swiss chabis weisser Kopfkohl; Brust. Cf. 2A14.

81B17. Swiss chern Fruchtkern; Brustdrüse, Bruststück; Milchdrüsen am Euter, Brust, NHG. kern.

81B18. EDial. clushet cow's udder. Cf. 104A4.

81B19. Cologne milchschaaf starke Frauenbrust.

81B20. Swiss nuggel Saugzapfen; weibl. Brust, Oberh. nückel Weiberbrust, Kurhess. kleine Erhöhung; weibl. Brust; der im Zorne hervorstehende Muskelteil der Stirn über den Augenbrauen, NHG. nock kleiner Hügel.

81B21. Westf. spondel Euter, spunder Euterstück; spund Zapfenloch; Euter, NHG. kurzer Verschlusszapfen.

81B22. Du. tepel round projection; teat, EFris. tepel Brustwarze, NE. tip.

81B23. Sax. vorbau weibl. Brust, NHG. bauen.

81B24. Wald. vortrag Busen, NHG. tragen.

81C1. Swiss plämpe Euter, plamper hangendes,baumelndes Ding, plampen schaukeln, baumeln, plämper Zitze. Cf. $101 \mathrm{~B} 3$.

81C2. Swiss hängerli Zitzen, Swab. b'henk weibl. Brüste. Cf. $25 \mathrm{D} 3$.

81C3. EDial. diddy female breast with milk in it, teat, nipple, didds teats of an animal, NE. didder tremble, shake. 
81C4. Steir. tilge Zitze des Mutterschweines; Röhre aus Eisenblech, in die ein Holzstiel gesteckt werden kann, Sax. tilchen Schneeglöckchen.

81C5. Bav. schumeln Brüste, schummeln wiegen, NHG. schlotterig einhergehen.

81C6. Swiss luller Lutschbeutel, Brustwarze, lullen saugen, die Zunge heraushängen lassen. Cf. 22A2.

81C7. Bav. lampen Zitzen; Stück vom Hemd, das aus dem Beinkleid niederhängt, Als. lämpe Hängebrüste. Cf. 140A8.

81CC1. Swiss flāme Euter, das anfängt zu schwellen; weiche, flaumige, zarte Haut; Nierenfett. Cf. 35A2.

81D1. NE. $d u g$ udder, teat, nipple, Swed. doegga suckle a child (NED.).

81D2. DuDial. lokkemam, lokketette mother's breast; sugar tit, lokken "slorp."

81D3. NE. nipple small terminating prominence of the breast, nip compress between two surfaces or points, pinch.

81D4. Lothr. nubel Brust, nubeln saugen, lutschen, NHG. nuppeln idem.

81D5. Swiss saug Zitze, sugeli Brustwarze, Zitze, Westf. sog Euter, NHG. saugen.

81DD1. NHG. strich stroke, line, streak; teat of a cow.

81DD2. Steir. nifen wunde Brustwarzen, Swab. niffeln reiben, wetzen auf schädl. Art.

81E1. Swab. gemelk Euter, Brust.

81F1. Du. elder udder, breast, Goth. alan aufwachsen, sich nähren (Franck).

81G1. EDial. drills teats, drillen dripping, drill drip, trickle.

81H1. Lux. nenn Brust, Als. nänne Mutter, Swab. näni idem.

81J1. NHG. peter und paul weibl. Brüste.

81J2. Swab. katarine Brüste.

81K1. NHG. potenz Macht; Zeugungskraft; Brust.

81L1. Swiss güeteli weibl. Brust; Kern der Nuss, NE. goody sweetmeat: good.

81M1. EDial. quarter one of the four teats of a cow, NE. fourth of anything.

81N1. Fris. by-ür extra teat of cow or sheep, ür udder.

81P1. Swiss brīmel weibl. Brust. 
82. Bosom

The bosom is described (A) as a heap, bunch; (B) as a burden; (C) as the heart and heart binder.

82A1. NE. bulk a heap; the gross; greater part; breast; chest, thorax, Dan. bump, knob, ME. bolke heap.

81A2. Swiss schīterbīg Holzstoss, Busen: schīter Scheit+bīg Stoss.

82A3. Swiss nest Busen. Cf. 5A42.

82B1. Swiss burdi Bürde; voller weibl. Busen. Cf. $85 \mathrm{C} 1$.

82C1. Swab. herz Herz; Busen; Brust; Magen.

82C2. Hess. herzbendel Brust, Brustbein, NHG. pericardium, MHG. bendel Band, Binde. Cf. 97A1.

\section{Thorax}

The thorax is described as a basket, box, chest.

83A1. Swiss bīcher Bienenkorb; Brustkorb: OHG. bīa Biene+ MHG. kar Geschirr, Schüssel, Bienenkorb, etc.

83A2. Swab. kreb Weidenkorb zum Tragen; Wagenkorb; Brustkorb. Cf. $44 \mathrm{C} 2$.

83A3. NHG. brustkorb thorax. Cf. 7F13.

83A4. NHG. brustkasten thorax, Swiss chasten idem.

83A5. NE. chest thorax, OE. cist box, coffin.

83A6. Lothr. kadaschter Brustkorb: kad(e) kräftig gebauter Mensch, kräftiges Zugtier+kaschten Kasten, or (kan)aster, Span. canastro reed basket. Compare following word.

83A7. Als. känster Küchenkasten; Brustkasten, Lat. canistra basket.

\section{WAIST}

The waist is described (A) as a girdle; (B) as the middle; (C) as a swelling.

84A1. Swiss gurt Gürtel; Gürtelgegend des Leibes.

84B1. NE. middle middle part of the human body, waist, Du. middel waist.

84C1. NE. waist part of the body between the chest and hips, OE. wast, waxt growth, fruit, produce. 


\section{BeLLY}

The belly is described (A) as a swelling, lump, and the like; (B) as something dangling, flabby; (C) as a burden; (D) as something flat; (E) as the part grasped (to test fatness. Cf. 66E); (F) as fat; $(\mathrm{G})$ as a skin; $(\mathrm{H})$ as a gurgler; $(\mathrm{J})$ unclassified.

85A1. Fris. aeikoer belly. Cf. 90A42.

85A2. Swiss amboss Amboss; Unterleib, OHG. anabōz anvil.

85A3. MLG., Hess. amen Bauchseite des Schweines, NE. awm, NHG. ohm, MLat. ama Gefäss, Ohm.

85A4. Swiss bantsch Schmerbauch; Bausch; Gepäck, Bürde sofern sie vorsteht, bantschen sich den Wanst füllen. Cf. panze Wanst, MHG. panze, Lat. pantex, Fr. panse idem.

85A5. Als. bibele hervorstehender dicker Bauch. Cf. 2B2.

85A6. Bav. pipsack Bauch, Wanst. Possibly related to the preceding.

85A7. Swiss bläster Dickwanst. Cf. 40A3.

85A8. MDu. bolle elongated round object; belly. Cf. $2 \mathrm{~A} 3$.

85A9. Steir. pude, bute Bauch; Last, Kärnt. pud'n Bauch, Swiss buderich dicker Bauch; kleiner dicker Mensch, budi grossbäuchiger Krug, Flasche; Wanst, budel Bauch an Gefässen; Bauch, MHG. büte, bütte Gefäss, Bütte. Cf. Swiss baudi Bauch, Magen; bauschige Figur and 113A4.

85A10. Schles. böttich, büttich, pitch Leib, Bauch, NHG. bottich hölzernes Gefäss, EDial. body abdomen. Compare Arnl. 94:07.

85A11. Westf., Sax. butten Bauch. Compare the preceding.

85A12. Swiss bütterich Gefäss; Hinterteil der Spinne, von Personen; Bauch und Hinterteil der Geflügel; OHG. butirih Schlauch, Gefäss. Cf. 101A3.

85A13. Swab., Swiss bätterich dicker Bauch. Rime word to the preceding.

85A14. Swiss bänserich Fettwanst: pans $(e)+($ bütt)erich.

85A15. EDial. boug stomach, belly, bougan large end of a piece of wood, NE. bouge bag, wallet; bilge of a cask.

85A16. Als. bulti dicker Bauch. Cf. 2B4.

85A17. Swiss bündel Bündel; Kropf am menschlichen Halse; schwangerer Leib des Weibes; Hodensack eines Katers, OHG. gibuntili bundle. 
85A18. Swiss buspi Magen; dicker Bauch; grossbäuchiger Krug.

85A19. NE. cod belly. Cf. 127B9.

85A20. Swab. tanister Tornister; Buckel; dicker Bauch, Czech tanistra Tornister.

85A21. Swab. gelte mittelgrosses Wassergefäss; grosser Bauch, NHG. hölzernes Gefäss.

85A22. Tirol. gund'l Tragbütte; Brust, Bauch, Kärnt. gundl scheibenförmiges Holzgefäss, Fr. gondole Trinkgefäss (Lex.). Cf. $81 \mathrm{~B} 13$.

85A23. Hess. günter Magen, Bauch, Wald. günterek dickstes Darmende des Schweines, Swiss gunte kesselartige Vertiefung; jede Ansammlung von Flüssigkeit. Cf. 114A2.

85A24. EDial. hag paunch, belly, haggis dish of the lungs, heart, and liver of a sheep minced and cooked in a sheep's maw; paunch, belly; chitterlings of a calf, verb bruise, cut up, hag hew, chop, hack.

85A25. EFris. holster ledernes Futteral zum Einstecken der Pistolen; Bauchhöhle, Wanst, Magen. Cf. 7F15.

85A26. Swiss hode Stelle am Unterleibe des Mastviehs, an der man die Fettigkeit prüft; übertr. auf Männer, Swab. hode Kartoffel, Swiss hödle kleine runde Birne. Compare Arnl. 142:26.

85A27. Flem. hot basket for the back; belly, Swab. hutte, hotte Rückenkorb; Düte, LG., Swiss hotte Rückenkorb, Tragbütte. Cf. 89A8, 132B1, 175B3.

85A28. Sax. kaldaunenkasten Bauch. Compare Arnl. 135:36.

85A29. EDial. keg belly, stomach, kedge idem, verb stuff, fill, adj. tight.

85A30. Swab. köz Rückenkorb; Zwerchsack; Gestell am Haberrechen; Höcker; Bauch. Cf. 89A10.

85A31. Als. chratten Wanst. Cf. 90A43.

85A32. Als. kräze Bauch. Cf. 89A11.

85A33. Swiss lieni Wanst, lienert ungeschickter, unordentlicher, nachlässiger Mensch (Schw.).

85A34. Flem. lier belly, WFlem. liere belly; sack. Cf. 15A2.

85A35. EFris. michel Magen, Bauch. Cf. 85A33, 91A9.

85A36. Swiss mutti Bauch. Cf. 7H20.

85A37. Swiss nest Bauch. Cf. 5A42. 
85A38. Fris. pampes, pampses thick belly. Cf. 9A5.

85A39. Kärnt. plunze grosser Bauch. Cf. 9A2.

85A40. EDial. pod large protuberant stomach; little person; small animal. Cf. 74B15.

85A41. EDial. poke bag; belly, stomach, Flem. poke, pook sack; belly, crop, ME. poke bag. Cf. 101A12, $129 \mathrm{~B} 1$.

85A42. Prus. präzel Bauch. Cf. 50C1.

85A43. Prus. prömmel starker, dicker Bauch, promsen dicht einstopfen, pröms, prems Mus, Mehlsuppe. Cf. 81B7.

85A44. Swab. quätte fetter, voller Bauch, OHG. chwadilla Hautbläschen.

85A45. Hess. quenzel dicker Bauch: qu(atsch) nasser, weicher Kot; zusammengesessene Speise $+(r) a ̈ n z e l$ Ledersack; Bauch. Cf. $113 \mathrm{~B} 7$.

85A46. Als. sack Sack; Bauch, Swab. katsack Wanst; MDu. sac, DuDial. zak belly, Swiss zersack idem. Cf. 1B3, 90A52, 102A1, $125 \mathrm{~A} 8$.

85A47. Kärnt. semper grosser Bauch oder Kropf, MHG. sumber Geflecht, Korb. Cf. 85A49, 101A13.

85A48. EDial. spring young growth; of pork the belly or lower part of a forequarter divided from the neck, verb bulge, burst.

85A49. Bav., Swiss sumber Korb; Krug mit grossem Bauch; dicker, hervorstehender Leib, Oberh. sömber dicker, hervorstehender Leib; grosse Trommel. Cf. 85A47, 90A54.

85A50. Wald. stalenpot dreibeiniger Kochtopf; Magen, Leib: stalen leg, support+pot pot.

85A51. DuDial. trommel thick belly, NHG. trommel. Cf. $90 \mathrm{~A} 57$.

85B1. Als. dudel Bauch; Kropf; Pfeife, düdlen langsam gehen und watscheln.

85B2. EDial. hodge paunch of a pig, stomach, belly, verb stagger, shake.

85B3. Swab. hudel Lumpen, Tuchlappe; Bauch, huder Lappe, Lumpen, Swiss hudlen schlottern, bammeln. Cf. 113C3.

85B4. MLG. lappe Fetzen; weiches Bauchfleisch der Tiere, OHG. lappa niederhängendes Zeugstück.

85B5. Du. leb flabby underside of fish. Cf. 8C6. 
85B6. Kärnt. seng'l, senk'l Glockenschwengel; grosser Bauch oder Kropf, Tirol. sengg'l grosser baumelnder Gegenstand; grosser Kropf.

85B7. Swiss nötter grosser, dicker Wanst, Swab. nottlen etwas hin und her bewegen; wackeln. Cf. 123A21.

85C1. Swiss burdi schwangerer Leib einer Frau. Cf. 82B1, $113 \mathrm{G} 1$.

85D1. MDu. pletse, plettzer, plette paunch of slaughtered animals, pletten make flat, EFris. pletske kleiner flacher Kuchen.

85E1. EFris. fangst Wanst, fangstuk Bauchfleisch. Cf. 140D1.

85F1. MDu. smeer fat; fat part; belly, Du. smeerbuik, NHG. schmerbauch thick belly, OHG. smero fat.

85G1. NE. rim membrane; peritoneum; intestines; belly, OE. rēoma membrane, ligament. Compare Arnl. 144:14.

85H1. Swiss güdel Bauch, Wanst, guderen gurgeln, kollern. Cf. $101 \mathrm{C} 1$.

85J1. Westf. tenterk Magen, Leib.

86. Pit of the Stomach

The pit of the stomach is described as a pit, hollow.

86A1. NHG. herzgrube pit of the stomach.

86A2. Cologne hätzeküülche Herzgrube: hätz Herz+küül Kaul, Grube.

86A3. MLG. hert-lepel Präcordium, lepel Löffel.

86A4. EDial. heartspoon pit of the stomach, spoon wooden shovel; navel, part of the breast.

86A5. DuDial. herteput, -pit pit of the stomach, NE. pit.

\section{NAVEL}

The navel is described (A) as a knob, lump; (B) as a hole.

87A1. DuDial. buiknagel, nagelbuik navel.

87A2. EDial. button, bellybutton navel.

87A3. Swiss krom navel, MHG. grome Hode, Lat. grümus Häufchen (Schw.).

87B1. EDial. bellyhole navel. 


\section{Umbilical Cord}

The umbilical cord is described (A) as a cord; (B) as a pliable stick.

88A1. Swab. leitseil Nabelschnur: leiten+seil.

88A2. DuDial. pezerik umbilicus. Cf. $123 \mathrm{E} 3$.

88A3. LG. streng Nabelstrang, NHG. strang.

88B1. Swiss, Swab. nabelgerte Nabelschnur.

\section{BACK}

The back is described (A) as a lump, bump, ridge; (B) as a roof, covering; (C) as that which sways, bends; (D) as a cross; (E) as something stiff; (F) as a keel; (G) unclassified.

89A1. Als. buckel Rücken, fehlerhafter Rücken, Höcker; Hügel, Swiss buggel, Du. bockel idem, NHG. buckel.

89A2. Du. bult Buckel. Cf. 104A2.

89A3. Thur. bürzel Rücken. Cf. 90A19.

89A4. Swiss guppel Rücken. Cf. 4C1.

89A5. Lux. hatzebatz der gekrümmte Rücken: hatze(len) schütteln, rütteln (Als. hotzel, hutzel Schaukel)+batz Kerngehäuse. Cf. $42 \mathrm{~A} 3$.

89A6. Lux. hatzebockel Rücken. Formed after 89A5.

89A7. Thur. hocke Haufen; Bündel; Rücken, Buckel, Sax. hocke, hucke Traglast; verwachsener Rücken, Prus. huck Rücken, Neumärk. huke Rücken; Haufen, NHG. hucke Rücken als Träger der Bürde. Cf. 5A37, 40A6.

89A8. Flem hot back. Cf. 85A27.

89A9. Swiss huppe der erhöhte Hinterrücken des Pferdes, NE. hub. Cf. 5A39.

89A10. Lothr. kotz Rückenkorb; Rücken. Cf. 85A30.

89A11. Als. krätze geflochtener Tragkorb; Buckel, Rücken, MHG. kretze Tragkorb. Cf. 45A3, 85A32, $175 \mathrm{~B} 4$.

89A12. Flem. krof, kroef hunchback; protuberance, Du. kroft, krocht stony elevation.

89A13. DuDial. mes back, same as older meerse, meerskorf market basket (Schu.).

89A14. DuDial. niknak back, shoulders, Kil. neck, nick, nack, NHG. nacken. 
89A15. DuDial. nok ridge of a roof; forepart of the back of a cow, Du. ridge, top; ridgetimber.

89A16. DuDial. stuit, stuut lower end of the backbone; hunchback, hump; projection, NHG. steiss. Compare Arnl. 112:24.

89A17. Swiss ranzen Felleisen; Rücken, Buckel; Ausbauchung an einer Mauer, EFris. randsel idem, NHG. ranzen.

89B1. Prus. dach Rücken. Cf. $2 \mathrm{G} 1$.

89B2. EDial. pilch back; thick shoulder shawl; anything hung before the thighs to protect them; saddle pad, OE. pylce, pilece robe of skin (Wr.).

89C1. Swab. gauke Rücken; Pumpe, gauken sich hin und her bewegen, schwerfällig schwanken; pumpen, NHG. gaukeln.

89D1. Sax. kreuz unterster Rücken des Menschen, NHG. Rückgrat zw. den Schultern od. am unteren Ende, Fris. krus, Lothr. kritz idem.

89E1. Prus. ragge Rücken; Pferd, Swiss gerăgel etwas Hartes, Beiniges; hageres Geschöpf, ragen starr, steif sein, EDial. rack neck or scrag of mutton; segment of backbone; bones of a dead horse; bony horse.

89F1. EDial. keel spine, lower part of the back. A comparison suggested by the spine with the ribs branching off.

89G1. Prus. rosak Rücken; Prügel, Hiebe.

\section{0. Вuтtocks}

The buttocks are described (A) as a rounding, swelling, lump, chunk, club, bolt, protuberance, bend, angle; (B) as the rear, back part; (C) as that which is beneath, bottom; (D) as that which is ridden, sat on, sits; (E) as a crevice, notch, hollow; (F) as the pudendum; $(\mathrm{G})$ as a noise-maker; $(\mathrm{H})$ as something prominent; (J) as something flat; (K) as a covering; (L) as something wiped; (M) as something ill-smelling; (N) as that which voids; (P) as something insignificant; ( $\dot{\mathrm{R}})$ as something bare, exposed; $(\mathrm{S})$ as that which is grasped; (T) as a feather; (U) as a kitchen; (V) as a gun carriage; (W) by proper names; (X) by euphemisms; (Y) by a contracted form; (Z) unclassified.

90A1. Swab. ecklein piece on each side of the root of the tail of beeves, NHG. ecke. 
90A2. DuDial. ende butt of a tree; of straw; bundle of grain inverted; buttocks, Kil. anus, sedes.

90A3. Lothr. bällerten buttocks; gums, Oberh. bellerchen gums, before the teeth come through, OHG. bilarn gums.

90A4. Westf. batsen buttocks; thigh, Cologne batze buttocks, NHG. batzen Klumpen. Cf. 42A3, 69A1.

90A5. Als. badutter Gesäss: ba(tterig) durch Nässe schmeirig, weich+dutter (90A29). Compare Wood, JEGPh., XI, 298.

90A6. DuDial. bastje rump of a slaughtered animal. Perhaps for bats, as in ZLimb. bats runderbil occurs. Cf. 90A4.

90A7. Swab. becher podex, OHG. behhar Becher.

90A8. Cologne bolle Hinterteil vom Veih, Wald. bole Hinterbacke. Cf. $26 \mathrm{E} 1$.

90A9. Du. botten buttocks. Cf. 151A1, 90A10.

90A10. EDial. butt buttocks; stump, etc., NE. buttock, ME. buttok one of the two protuberances of the rump. Cf. 151A1.

90A11. Swiss bütterich Hinterteil beim Geflügel. Cf. 85A12.

90A12. Lux. breissem Gesäss, Swiss brässmen Schamgegend, brassmen breeches, NHG. brasem, brachsme, OE. brēc breech, hindquarters. For OE. brēc cf. Wood, Mod. Phil., XI, 327.

90A13. Swiss büchs Büchse; Hinterer; After-Scheide von Tieren, Swab. Podex mit Anklang an Schiessgewehr. Cf. 127B1.

90A14. EDial. buckie hindquarters of a hare. Cf. $69 \mathrm{C1}$.

90A15. Swiss budi Kosename für einen kleinen dicken Hund, Knaben; Gesäss der Kinder. Cf. 5C4.

90A16. NE. bum buttocks, Swiss bummi idem. Cf. $123 \mathrm{C} 8$.

90A17. EDial. bump buttocks; hillock, NE. swelling, etc. Cf. 5A8.

90A18. Prus. bomst podex; Fell: $\operatorname{bom}(s)$ anything very large + (ba)st hide. Cf. 132A1.

90A19. NHG. bürzel Steissende, dial. berzel, börzel, borzen, burz, burzer buttocks; rounding protuberance; swelling; small hill, etc., OHG. burian in die Höhe halten oder recken. Cf. 2B5, 5A2, 89A3, $127 \mathrm{~A} 2$.

90A20. Bav. füdlein, Westf. fuet buttocks, MHG. füdel mons veneris. Compare Arnl. 143:42.

90A21. Sax., Als. fetzer Hinterer, fetzen grosses Stück, Swab. fetz ungeformtes Stück. 
90A22. DuDial. center podex, cinter, center wooden arch, Fr. cintre idem.

90A23. EDial. curriehunkers hams: currie small stool +hunker squat with the knees, haunches and ankles acutely bent.

90A24. Thur. deise Hinterer, DuDial. doos idem. Cf. 127A24.

90A25. DuDial. doel dreutel, hoopje drek; ardhoop; aars, MLG. dōle gracht, groeve met de daarnaast opgehoogde aarde (Franck). Cf. 90A34.

90A26. EDial. dock solid, fleshy part of an animal's tail; nether end of the body: of a felled tree, docksy broad nether end of the human body, Icel. dockr short stumpy tail, Fris. dok bundle, bunch, etc. (NED.).

90A27. EDial. doup end of an egg; buttocks, seat; bottom, end of anything; bay in a lake, recess, ON. daup? (NED.). Cf. 9 A16.

90A28. Prus. dups, duppe podex, poln. dupa Loch (Frisch.). Compare rather MLG. doppe Schale, Kapsel, Kelch, Topf; Knopf (Wood).

90A29. Als. dutter, bluttdutter Hinterer; Eidotter; aufgebundener Haartracht: blutt bloss+dutter Dotter.

90A30. EDial. driddles buttocks, driddlins knots of wet meal, driddle let fall in small quantities. Cf. 113A4a.

90A31. EDial. droddum the breech, drod short, thick, clubbish person; rude candlestick. Cf. preceding.

90A32. Swiss güggen buttocks. Cf. $123 \mathrm{C} 15$.

90A33. Swiss gutsch buttocks; hill. Cf. 2B10.

90A34. WFlem. kwadoel sluggish and flabby person; buttocks: MDu. qua(dele) pimple, boil+WFlem. doel (90A25). For this and the following words compare Wood, JEGPh., XI, 312.

90A35. Als. quadeterle buttocks: Swab. qua(ttle) dicker, fetter Junge mit watschelndem Gang+Als. *deterle weiche Masse (dätterig zitternd; weich, teigig). Cf. preceding.

90A36. Als. quadutter Hinterer; breites Hosengesäss: Swab. qua(ttle) $90 \mathrm{~A} 35+$ dutter $90 \mathrm{~A} 29$.

90A37. Swab. heie pile-driver; , hölzerner Hammer; Hinterer, MHG. heie Ramme.

90A38. Cologne huppet Hinterer, NHG. hoppe Erhöhung, Anschwellung, Bav. hopper eine kleine Erhöhung, Unebenheit. 
90A39. EDial. hurdy buttocks; hips and parts adjacent, hurder heap of stones, hurd hoard, NE. hurdies buttocks. Of unknown origin (NED.).

90A40. Swiss chäfi Käfig; Hinterer; dicker Teil der Nähnadel, MHG. kevje Vogelkäfig; Gefängnis.

90A41. Fris. kakwangen buttocks: kak stercus, drek+wangen.

90A42. Fris. koer basket; buttocks, aeikoer egg basket; podex; corpus, DuDial. korre idem. Cf. 1B1, 85A1.

90A43. Swiss chratte bauchiger Korb; Rundung des Leibes; Hinterer, MHG. kratte Korb. Cf. 85A31, 104A10, $175 \mathrm{~B} 5$.

90A44. DuDial. kröte podex, perhaps related to 175B4.

90A45. DuDial. kriek podex, zich een kriek lachen double up, hump up with laughter.

90A46. Fris. krint podex, krinten pimples, krintekoer achterste: krint currant+koer basket.

90A47. Swab. laib Laib Brot; Partie am After des Rindviehs.

90A48. Fris. ljirre smoked meat, thigh; buttocks, OE. līra brawn. Compare Arnl. 153:04.

90A49. EDial. mug rump of an animal. Cf. $7 \mathrm{H} 19$.

90A50. NE. natch buttocks, rump, var. of notch? (NED.), nache point of the rump of an $\mathrm{ox}$, rump, OF. nache rump.

90A51. Swiss nest Hinterer. Cf. 5A42.

90A52. Swiss sack Hinterer. Cf. 85A46.

90A53. DuDial. slot Buttocks, rump, Du., MDu. inclosure; prison; end.

90A54. Swiss sumber von einem dicken Hintern. Cf. 85A49.

90A55. NE. toute buttocks, ME. toute, tūte, OE. *tūte. Ger. *tūt- stick out, project (NED.), ON. tūta projecting point, tota point, etc.

90A56. EDial. tut buttocks, tutty hummocky. Cf. 19F2. Considered a var. of $90 \mathrm{~A} 55$ by NED.

90A57. DuDial. trommel achterste; short, thick woman. Cf. $85 \mathrm{~A} 51$.

90A58. Nos. tuppert Mörser; Hinterteil der Hose; podex, NE. tup ram; pile-driver.

90B1. Swab. aberschanz (rear fortification) buttocks.

90B2. (Meaning buttocks in general unless otherwise indicated). Du. achterste, DuDial. achterend, Fris. efterein, DuDial. achterborst 
(after-breast), achterpoort (after-port), achterwark (after-works), Du. achtergestel (after-frame) rump of a horse, DuDial. achtergelent (after-balustrade), achterkastijl (after-castle), achterhesp after-hasp. Cf. 9Dd.

90B3. NE. backside buttocks.

90B4. EDial. behind buttocks.

90B5. DuDial. hinderste, NE. hinderend, hindend, Sax. hinterbrust, hinterpummern (pum $[$ sen $]$ einen Schall geben $+[$ Pom $]$ mern), hinterviertel, hinterteilchen, hintergestelle, Zwickau hinterkhastel, Sax. hinterkammer, Swab. hinterquartier, NE. hindquarters (of animals) buttocks.

90B6. NE. stern hind part of a boat; buttocks, ME. sterne rudder.

90C1. Lothr. dockes Gesäss, other dial. dogges, douckes, dōkes, doches, tuches, DuDial. tokes idem, durch Missverständnis aus hebr. Präpos. tachath "unter" entstanden (Foll.).

90C2. NE. bottom buttocks; lower or hinder part of the trunk of an animal.

90D1. Swiss gereit Reitzeug; worauf jedermann sitzt; Hinterteil des Menschen, MHG. Reitzeug.

90D2. Swiss sitz, Flem. zit, MDu. set Sitzung; Gesäss, NE. sit.

90D2a. Swiss sessel wie nhd.; Steissbein.

90D3. NHG. sitzer wer sitzt; Gesäss.

90D4. NE. seat thing on which to sit; buttocks, NHG. gesäss.

90E1. Swab. graben Arschkerbe, NHG. graben.

90E2. Swiss chele Kehle; Vertiefung zw. den Hinterbacken; Rinne, MHG. kele Kehle.

90E3. Als. kerb Afterfurche; podex, Bav. kerben idem, Swiss arschkerb, fudlekerb, Swab. kerfe, kerbe, Wald. kierwe idem, NHG. kerbe.

90E4. Fris. kerne notch, crack; notch of the buttocks, kernje notch.

90E5. Bav. kluft Spalte; Ritze; Spalt hinten, NHG. kluft.

90E6. Swiss arschchrinne Spalte zw. den Hinterbacken, MHG. krinne Einschnitt, Kerbe, Rinne.

90E7. Swab. laffe Gesässspalte, Hinterteil. Cf. 46A2.

90E8. Swab. pfanne flaches Gefäss; Gesäss. Cf. 7H8, 127D1, 149B1. 
90F1. Swab. bos Hinterer, Hebr. bōš sich schämen (F.).

90F2. DuDial. treter achterste, treteren plagen, bitter spotten. 90F3. Swiss puntenöri point d'honneur; Hinterer, öri ist an öri Handhabe an Gefässen angelehnt (Schw.).

90F4. Lothr. urzel Bürzel, Steiss des Geflügels, Swiss urzen Speisereste; etwas Verwerfliches, MHG. urez des Essens überdrüssig.

90G1. Als. bupper Hinterer; Farz, bupp Farz, buppen cacare.

90G2. Altenb. puparsch, EFris. pupeers, Westf. puppert, DuDial. poeper, popper, poep, poepgat, Thur. pfopfe buttocks, Thur. pfopfen mit einer geringen Erschütterung fallen.

90G3. EDial. drone backside, breech, NE. continuous deep monotonous sound of humming.

90G4. DuDial. preut buttocks, MDu. prōtelen idem. Cf. $127 \mathrm{~N} 1$.

90H1. Wald. olerwärteste Hinterer, NHG. allerwärts.

90J1. Fris. brits breech; buttocks. Cf. 7H5.

90K1. Prus. portken Hosen; Hinterer, poln. portki Hosen (Frisch.). Cf. 68E1.

90L1. Swiss rībhart podex, rīben reiben.

90M1. Lux. hiewerling Steiss, hiewerlengsche keis Herver Käse.

90N1. Sax. scheisserle Hinterer, Du. schijtgat, Fris. skytgat, -lok idem.

90P1. EDial. petty rump; privy, NE. small, insignificant.

90R1. EDial. nacky nursery term for a child's fundament, nackt naked.

90R2. EDial. nally as 90R1. Probably a blend of na(cky)+ (be) lly.

90R3. Wald. blik Arsch. Cf. 92G1.

90S1. Fris. fang rump of a cow, Du. vang fleshy part of a cow's thigh, EFris. fangst idem, Du. vangst catch, catching. Cf. 66E1, 85E1, $140 \mathrm{D} 1$.

90S2. EDial. catch point of the rump. Cf. 66E1.

90T1. Swiss schwanzfedere Schwanzwurzel, Steissbein der Kuh, Hinterer. Vergleichung des Rückgrates sammt den von ihm seitlich auslaufenden Rippen mit dem Kiel und den Fasern einer Feder (Schw.), federen Rippen-, Schwanzstück des Fleisches von vierfüssigen Tieren.

90U1. Swiss chuchi Küche; podex. Cf. 104C1. 
90V1. Swiss, Als. lafete gun carriage; Hinterer, Als. idem, eine lafete machen to spread the legs apart and bend forward, Fr. l'affut Gestell einer Kanone. Cf. 6C4.

90W1. Bav. herodes podex; fliessende Hämorrhoiden.

90W2. Lux. krakau Steiss. Probably with play upon krachen.

90X1. Sax. die vier buchstaben Hinterer, Swab. buchstaben idem, für $p-o-p-o(\mathrm{~F}$.$) .$

90X2. Sax. dreier Zahl 3; Hinterer, NHG. die drei buchstaben idem, $a-r-s$.

90X3. Swab. feierabend der entblösste Hintere.

90X4. DuDial. flag podex, cf. Amer. star-spangled banner idem.

90X5. EDial. natty fundament, natomy anatomy.

90X6. DuDial. azent buttocks. Cf. 6C1.

90Y1. EFris. mars podex, Prus. marsch, DuDial. moars, maars idem, from $m($ ast $)+$ ars.

90Z1. Fris. efterkedet, efterketier podex.

90Z2. Cologne flöösch Hinterer.

90Z3. Als. näfzer After.

\section{Anus}

The anus is described (A) as an opening, eye; (B) as a bunch, pucker, end; (C) as a vein, tube, funnel.

91A1. Sax. auge podex.

91A2-3. Du., Fris., Prus. gat Loch; Hinterer, MDu. idem. Cf. 1G1, $127 \mathrm{C} 5$.

91A4. DuDial. hol anus, MLG. nedder-, achterhol idem.

91A5. Swiss chämin anus. Cf. 7F12.

91A6. Du. kont anus, Fris. kont, kunt idem. Compare Arnl. $143: 03$.

91A7. Flem. kuit, kut anus, Als. kuttele Grübchen in den Backen; podex. Cf. $127 \mathrm{C} 8$.

91A8. Ger. dial. loch, weideloch, puplach, finsterloch, finsterlink, fistloch anus, OHG. loh hole, etc.

91A9. Prus. mechile, -o Hinterer, podex, Hebr. mechillo Höhle, Höhlung (Frisch.). Cf. $127 \mathrm{C} 14$.

91A10. MDu., Antwerp put hole; anus. 
91B1. Swiss ros Rose; Schnuppe am Docht; horse's anus. Cf. $32 \mathrm{C} 2$.

91B2. DuDial. stropsel aars, strop puckering string; sphinctor ani.

91B3. EDial. tib anus. Cf. 25D5.

91C1. NHG. farzader podex.

91C2. Lothr. spektif Fernrohr; Hinterer, from perspektif (Foll.).

91C3. Lux. tūt Rohrartiges; podex, LG. tūte Trichterförmiges.

\section{TAIL}

The tail is described (A) as a club, bat, etc.; (B) as a protuberance, lump, stump; (C) as a bush, tuft; (D) as a coil, curl, curve; (E) as a roll, roller; beet; (F) as something hanging, dangling; (G) as something flashing, gleaming; $(\mathrm{H})$ as a feather; $(\mathrm{J})$ as a flower; (K) as a sting; (L) as a wheel; (M) as a lip; (N) as something undivided; $(\mathrm{P})$ unclassified.

92A1. Swiss bengel Kuschwanz. Cf. 123B1.

92A2. Thur. britsch Pritsche, Schwanz, Ballenb. britsche Schwanz des Ochsen. Cf. $7 \mathrm{H} 3$.

92A3. Als. gerte Gerte; Schwanz des Pferdes ohne Haare.

92A4. Swiss ruet Rute; penis; Schwanz. Compare Arnl. 141:14.

92B1. Lux. bischofs-kap mitra; Hinterteil des Huhnes.

92B2. Swiss birn vorderster Teil des Schwanzes beim Vieh. Cf. $123 \mathrm{C} 4$.

92B3. NE. bun tail of a hare; buttocks. Compare Gael. bun stump, root and $92 \mathrm{~B} 4$.

92B4. EDial. bunt tail of a rabbit; bagging part of a sail, of a fish net; small faggot of wood, NE. verb swell out, belly.

92B5. EDial. runt anything stunted in growth; stump of a tree; short thick stick; tail, esp. upper part of it; rump.

92B5a. Swiss rübe root of the tail. Cf. 2 A32.

92B6. Lux. rupp kurzer Schweif, Siebb. ruppen rupfen, NHG. rupfen.

92B7. EDial. scut tail of a hare; hare; tail, scutty short-tailed; stumpy, verb crouch down. Cf. 40A14.

92B8. Steir. stoss wie allg.; Schwanz des Auer-, Birkhahnes, Fasans und verschiedener Raubvögel, NHG. aufgeschichteter Haufen. 
92B9. EDial. strunt anything cut short or stunted; denuded root end of the tail, verb cut short, dock the tail of a horse.

92C1. Swiss besem Besen; Haarbüschel am Ende des Kuhschwanzes, Als. besen idem, OHG. besamo Besen.

92C2. NE. brush small trees and shrubs of a thicket; tails of some animals, as the fox.

92C3. Swiss fausel Büschel; Quaste; Haarbüschel am Schwanzende der Rinder.

92C4. Swiss fotzle Quaste; Tannzweig mit Nadelbusch; Haarbüschel am Schwanze des Rindviehs. Cf. 5A12.

92C5. Als. bensel, bemsel Pinsel; Schweif des Pferdes, Swab. pemsel Malerpinsel; Haarbüschel, Swiss bensel Pinsel.

92D1. EDial. quirk twist, bend, circle; end of a pig's tail, verb turn quickly, quirkle twist.

92D2. Swiss rigel sich ringelnder, kurzer Schwanz. Cf. 5B11.

92D3. Swiss, Bav. ring ring; pig's tail.

92D4. Als. sabel Säbel; gebogener Schwanz einer grossen Heuschreckenart.

92E1. Lothr. wichen, Als. wiechen Lampendocht aus gedrehten Fäden, Schwanz des Schweines, MHG. wieche, wicke Docht von Garn gedreht.

92E2. NHG. leier Kurbel; Schwanz der Sauen, nach der Aehnlichkeit mit einer Kurbel (Gr.).

92E3. Westf. mōr Schwanz von Kühen und Pferden; Möhre, OHG. moraha Rübe.

92F1. EDial. flip-by cow's tail, flip stroke with a whip, etc., fillip, verb strike lightly, fillip.

92F2. EDial. tag any small object hanging from a larger one; tail; white hair on the point of the tail of a cow; tail end of a rump of beef; twisted and pointed end of a lock of wool as it is shorn from the fleece.

92F3. Sax. klunker lange Schwanzquaste der Kuh. Cf. 53B2.

92G1. Wald. blik kurzer Schwanz der Ziegen, Hasen, Hirsche, MHG. blic Glanz, Blick, Blitz. Cf. 90R3.

92G2. NHG. lunte Zündstrick, -lappen; Fetzen, Lappen; Schwanz des Fuchses, Luchses, nach der brennend roten Farbe (Wg.). 
92H1. Swab. feder Feder; Schweif. Cf. 63A2, 174A1.

92H2. Du. pluim feather; hare's tail, Lat. pluma.

92J1. Swab. blum Blume; Schwänzchen des Hirsches. Cf. $127 \mathrm{X} 1$.

92K1. NE. sting fine taper of a dog's tail. Cf. 148A4.

92L1. Swiss rad Rad; aufgerichteter Schweif des Pfauhahns.

92M1. Swab. lippe Schwänzchen der Ziege, NHG. lippe.

92N1. EDial. single tail of a deer. Cf. 60D1, $113 \mathrm{~N} 1$.

92P1. Swab. elemost Schwanzstück des Schweines.

93. FLaNK, Side

The side is described (A) as a hollow; (B) as the pudendum.

93A1. DuDial. miltepit, -put left flank of a cow: milten tap a cow swollen with gas + pit.

93A2. Bav. wannen flank, NHG. wanne länglich-rundes Gefäss zum Waschen; Höhlung zw. Hinterschenkel und Bauch, MHG. langrundes Gefäss zum Backen.

93B1. Oberharz. schamm Seite unter dem Blatte.

94. LoIN

The loin is described (A) as something round; (B) as a roast; (C) as a cleft; (D) unclassified.

94A1. Wald. bol Schenkel, Lende. Cf. 2 A3.

94B1. Fris. harste loin of beef, Du. harst roast, MDu. harsten to roast.

94C1. Wald. kluft Lende, kluftstück Stück Fleisch aus der Lende, NHG. kluft abgespaltenes grosses Holzstück. Cf. 90E5.

94D1. DuDial. lee loins.

94D2. Cologne schäsch Nierstück vom Rindvieh.

95. LAP

The lap is described as a flap.

95A1. Als., Lothr. geren Schoss; keilförmiges Stück, Ruhl. garn Schoss, MHG, ger, gere Wurfspiess; keilförmiges Zeugstück, das unten an ein Gewand eingesetzt ist. Compare Arnl. 99:01.

95A2. NE. lap part of the body covered by the front of the skirts, OE. lappa skirt, lappet. 
96. Heart

Swiss nest Herz. Cf. 5A42.

\section{Pericardium}

Hess. herzbendel, Ruhl. härzbängel ( $n g$ for $n d$ ) pericardium. Cf. 82C2.

\section{Valve of the Heart}

Du. klapvlies (clap membrane) valve of the heart.

\section{LUNG}

The lung is described (A) as a bellows; (B) as something light;

(C) as something loose; (D) unclassified.

99A1. Sax. blasebalg Lunge; bellows.

99B1. Flem. licht lung; liver, NE. lights lungs. Cf. 129L1.

99C1. Du. loos lung, adj. loose, free.

99D1. EDial. flungs lungs. Blend of lungs +?

\section{KIDNEY}

The kidney is described (A) as a lump, swelling; (B) as a rich man.

100A1. Lux. eirchen Niere, NHG. ei.

100A2. Fris. stutsje kidney, stut oblong loaf; podex, OHG. stiuz buttocks. Compare Arnl. 112:24.

100A3. Fris. prōlle, prōlling kidney. Cf. 8A3.

100B1. Fris. rikeman (rich man) kidney surrounded by fat.

\section{Crop}

The crop is described (A) as a swelling, pouch, lump, projection; (B) as a dangle; (C) as a gurgler.

101A1. Swiss bibi, bippi, bippeli kleine Eiterpustel; Kropf. Cf. $2 \mathrm{~B} 2$.

101A2. Swiss blāst kleiner Kropf. Cf. 40A3.

101A3. Swiss bütterich strotzender Kropf. Cf. 85A12.

101A4. NE. craw crop; stomach, OHG. chrago neck, throat (NED.). Compare Arnl. 41:02. 
101A5. EDial. creak bird's crop, NE. creek, creak recess, inlet in the seacoast; turn, winding, as of a river, crooked way; applied to the two cavities of the heart.

101A6. Swab. deiei Kropf: dei längliches Brot+ei.

101A7. Swiss gōl Kropf; grober Steinschutt, goleten rough heap of stones; steile gepflasterte Strasse.

101A8. Swiss gutter bauchige Glasflasche; Kropf; Blase; Eidotter; Swab. dickbauchige Flasche.

101A9. Steir. jodel Lümmel, Tölpel; Stier; 'Kropf.

101A10. Prus. kader Kropf. Cf. 14A10.

101A12. Flem. poke crop. Cf. 85A41.

101A13. Kärnt. semper grosser Kropf. Cf. 85A47.

101B1. Steir. gagel Kropf, Swiss gagen hin und her schwanken, gagi Person, die gern auf einem Stuhle schaukelt. Cf. 126B1.

101B2. Swiss ginggel Kropf als schwappelnder. Cf. 123A8.

101B3. Swiss plämpe Kropf, plumpe Glockenschwengel; grosser Kropf. Cf. $81 \mathrm{C} 1$.

101B4. Kärnt. seng'l Kropf. Cf. 85B6.

101B5. Bav. waderer Kropf, MHG wadelen unbeständig sein, schwanken, NHG. wedeln.

101C1. Swiss güdel Kropf. Cf. 85H1.

101C2. EDial. clock crop, verb cluck, gurgle. Cf. 24D1.

\section{Gall Bladder}

Swiss sack Gallenblase. Cf. 85A46.

\section{Gizzard}

EDial. quern gizzard; small stone handmill, OE. cweorn, cwyrn handmill.

\section{Sтомасн}

The stomach is described (A) as a round object, swelling, lump, thick object; (B) as a receptacle, container; (C) as some familiar household article; (D) as something soft; (E) as something hanging; (F) as something rough; $(\mathrm{G})$ as a slobberer; $(\mathrm{H})$ unclassified.

104A1. EDial. bole ball-shaped stomach of the crab, OE. bolla any round vessel (Wr.). 
104A2. Swiss bulze mit Blut gefüllter Schafmagen; Anschwellung; kleine Erhöhung, MLG. bulte Hügel, Haufe. Cf. 89A2.

104A3. Swab. buntner, bunt Magen eines Tieres. Cf. 127A5.

104A4. EDial. clushet sow's stomach, clushan dung of a cow in a small heap. Cf. $81 \mathrm{~B} 18$.

104A5. NE. craw stomach. Cf. $101 \mathrm{~A} 4$.

104A6. EDial. crop stomach; breast; head; neck; throat. Compare Arnl. 127:03.

104A7. DuDial. vlint, flent stone; cow's stomach, because it resembles a cobblestone.

104A8. Als. hund Hund; Darmsack im Schweinemagen, welcher mit Blut gefüllt und gekocht.wird, hund füllen sich voll fressen.

104A9. EDial. jot calf's maw; paunch; breech; heavy article, adj. plump, verb strut.

104A10. Als. kratte runder Korb; Magen, Wanst. cf. 90A42.

104A11. Lux. mettei Magen; etwas Schwerfälliges. Probably same as Mettei Matthias.

104A12. DuDial. mook cow's stomach; fat woman, moker heavy hammer.

104A13. Wald. stalenpot Magen, Leib; stalen (69B10)+pot.

104B1. EDial. basket, breadbasket stomach.

104B2. EDial. creel basket; stomach.

104B3. NE. bag, baggie stomach, EDial. geordybag idem., geordy guinea. Cf. $81 \mathrm{~A} 1$.

104B4. Fris. knapsek knapsack; stomach, Fris. ropsek hungry stomach, roppich greedy, hungry.

104B5. Westf. löer Magen, Darme, Hess. lüre, lore Schlauch, OHG. lüdara culeus, saccus (Wo.).

104B6. Swiss choslup-molan, -malen Kälbermagen als Käslab verwendet, molan Tasche, Felleisen.

104B7. Als. grempenladen Laden eines Kleinhändlers; Magen, gremper Kleinhändler.

104B8. DuDial. lerze, leerze, laars boot; in jest, stomach.

104B9. EDial. muggie stomach of a fish. Cf. 7H19.

104B10. EDial. truncheon stomach, truncher trencher, NE. truncheon.

104C1. Swiss chuchi, Swab. kuche Küche; Magen, ESlang kitchen stomach. Cf. 90U1, $127 \mathrm{U} 1$. 
104C2. EDial. churn stomach.

104D1. EDial. kite, kytock, NE. kyte stomach, paunch. Cf. $113 \mathrm{~B} 5$.

104E1. DuDial. lap tripe stomach. Cf. 8C6.

104F1. Du. rob stomach of a big fish, Fris. robbe idem, robbich rough, uneven. Cf. Lith. rùpas rough (Franck). Cf. 113F1.

104G1. DuDial. slabbaris stomach, slabber bib; slobberer. From slabb(eren) slobber, "slorp"+? Cf. 7C3.

104H1. Wald. pije Magen.

\section{OMasum}

The omasum is described (A) as something like a book, having or composed of leaves; (B) as composed of folds; (C) as a rag.

105A1. EDial. bible-tripe third stomach of a ruminant, so called from the many folds or layers like the leaves of a book (Wr.), Prus. fibel Magen der Wiederkäuer; Psalter, Buch.

105A2. EDial. book-tripe third stomach of a cow.

105A3. Du. bladmaag, NHG. blättermagen booktripe.

105A4. Du. boekpens booktripe.

105B1. EDial. feck cow's stomach, variant of faik fold; manyplies, verb fold, tuck up (NED.). Cf. 105B2.

105B2. EDial. fack stomach of a ruminating animal, EFris. fak Fach, NHG. einfach etc.

105B3. EDial. manifolds omasum; intestines: many+folds.

105B4. NE. manyplies, EDial. moniplies omasum: many + ply. 105C1. Als. bletzer Blättermagen; Kaldaunen. Cf. 127F2.

\section{Aвomasum}

The abomasum is described (A) as that which causes coagulation; (B) as fat; cream; (C) as something curly, waving.

106A1. Du. leb abomasum; rennet, DuDial., EFris. lebbe, Fris. lēbbe, Prus. lāb, lāf idem, NHG. lab Mittel zum Gerinnenmachen, OHG. liberóon gerinnen.

106A2. NE. rennet fourth stomach of ruminants, OE. gerinnan curdle.

106B1. NHG. fettmagen fourth stomach of ruminants. 
106B2. Swiss raum Rahm, Sahne; Haut, Decke auf gekochter Milch; abomasum, NHG. rommagen Labmagen, MHG. (milch)roum fetter Abhub der Milch.

106C1. Swiss reide abomasum, reid gekräuselt, MHG. reit idem.

\section{Peritoneum}

The peritoneum is described.(A) as a sack; (B) as the belly skin; (C) as a net; (D) as a hood; (E) as that which carries; (F) as dead flesh.

107A1. Thur. bors Bauchnetz der Schweine, Swiss borsen, borsel, Rheinl. bōrsche cow's peritoneum. Cf. 113H1.

107B1. Du. buikvlies peritoneum: buik+vlies.

107C1. Swiss netzi, Als. netz peritoneum, NHG. netz.

107D1. Du. huif hood; peritoneum, NHG. haube.

107E1. Swiss burdi peritoneum. Cf. 113G1.

107F1. Swab. todfleisch peritoneum. Cf. 110C1.

\section{Pleura}

The pleura is described as the rib skin.

Du. ribbenvlies pleura.

\section{GLand}

The glands are described (B) as milkers; (C) as curls, lumps, swellings; (D) unclassified.

109B1. Swiss milchling Brustdrüse; Samenmilchdrüse. Cf. $111 \mathrm{~B} 1$.

109C1. Wald. stoüt oil gland of waterfowl, Du. stuitklier idem: stuit hump, hunchback+klier (109C2).

109C2. Du., Fris. klier, Scotch clyre, EFris. klire gland, MDu. cliere gland; swelling, Kil. kliere ulcer of the inguen; tumor; bubo venereus, probably connected with MDu. clydere swelling (Franck), Du. botan. klier boll.

109C3. EDial. curls glands of the neck, NE. curl.

109D1. NHG. midder Drüsen am Kälberhals.

\section{Pancreas}

The pancreas is described (A) as a lump; (B) as something sweet; (C) as dead flesh. 
110A1. EDial. breeds pancreas, NE. sweet-bread idem, OE. brēad fragment, bread. Cf. 110B1.

110A2. EDial. burr pancreas; rubblestone, NE. burr plant; protuberance more or less irregular in form.

110B1. Flem. zeutsel, Aachen 'süsschen calf's pancreas. Cf. $110 \mathrm{~A} 1$.

110C1. Swiss totenfleisch Milz, die blutreiche Milz hat Aehnlichkeit mit Fleisch, in welchem das Blut gestockt ist. Cf. 107F1.

\section{Thymus}

The thymus is described (A) as a lump; (B) as made of milk; (C) as a sweater; (D) as that which crumbles.

111A1. Swab. knauter Höcker; Halsdrüse; verkrüppelter Baum, verwachsener Mensch oder Tier, knäutel geschwollene Drüse; Geschwulst, Swiss chnüder Knoten, Knorren, etc.

111B1. Swiss milchen Brustdrüse; Kälberbröschen, milchling idem, Sax. kalbsmilch, Swab. milcher idem. Cf. 109B1.

111C1. EFris. sweterke, LG. sweder thymus, EFris. sweten schwitzen, NE. sweat, Du. zwezerik, EFris. sweser thymus may be related. Franck connects with Du. zuster, zwager.

111D1. Swab. brüslein Teig aus Mehl und Ei, der auf dem Reibeisen zerrieben ist; thymus, Bav. brües thymus, NHG. brosam das inwendige Weiche vom Brot, OE. brȳsan zerreiben. Compare Arnl. 135:29 for Swed. kalvbress, Dan. brissel thymus, Steir bris, brisel, bries, briesel, Ballenb. bries idem.

\section{Diaphragm}

The diaphragm is described (A) as something light; (B) as a net; (C) as a covering; (D) as something hanging, loose; (E) as a border, division; (F) as the heart blade; (G) unclassified.

112A1. Steir. lichtfleisch Zwerchfell der Rinder.

112B1. Wald. nets Zwerchfell der Tiere. Cf. $107 \mathrm{C} 1$.

112C1. EDial. apron diaphragm of animals; skin covering the belly of a roast duck or goose; abdomen of crustaceans, NE. piece of apparel for covering the front of a person; fat skin covering the belly of a goose. 
112D1. EDial. skirt diaphragm of animals; trimmings, loose pieces taken off the carcass of animals after being dressed; loose pieces of wool mixed with dung on a fleece, skirting diaphragm. Cf. $5 \mathrm{M} 3$.

112E1. Swiss līst Zwerchfell. Cf. 66C1.

112F1. NHG. herzblatt Zwerchfell; das Innere der Pflanzen.

112G1. DuDial. kraai midriff, NE. crow mesentery of animals, MHG. krā Gekröse.

112G2. Bav. kron, kronfleisch Zwerchfell beim Rindvieh. Cf. 79D1.

\section{INTESTINES}

The intestines are described (A) as a swelling, lump; (B) as a soft mass; (C) as something hanging; (D) as something stringy; (E) as something within; (F) as something plucked out, cleansed away; (G) as a burden; (H) as a sack; (J) as something unsound; (K) as something wound about; (L) as noise-makers; (M) by blends of words of similar meaning; (N) as something undivided; (P) unclassified.

113A1. LG. bolingh intestine, Du. bolling codfish, NE. bulk mass.

113A2. MDu. bulge, gebulchte, -balchte swelling; intestines, balch idem; belly. Cf. $1 \mathrm{~A} 2$.

113A3. EDial. pudding entrails, Westf. puddek lump, LG. puddig thick, stumpy (NED.).

113A4. Steir. pude, bute entrails. Cf. 85A9.

113A4a. EDial. driddles intestines. Cf. 90A30.

113A5. Swiss mausch Teil der Eingeweide beim Rindvieh. Cf. $2 \mathrm{~B} 12$.

113A6. EFris. pinke Darm. Cf. 58A2.

113A7. EDial. warisons bowels, warrison stomach and its contents, ME. warison, wareson protection; treasure, store.

113A8. Als. wurst Eingeweide; wulstig Gewundenes.

113B1. Swiss putzer weiche Masse; dicker Brei; Eingeweide von Insekten, NHG. butzen Klumpen; verdickte Feuchtigkeit in Nase, Auge.

113B2. Du. grom fish entrails; dregs, MDu. grom, Fris. grom, gram idem, Westf. grummelig klümperig. Cf. 126A10. 
113B3. EFris. grōs Eingeweide; Schmieriges, grōsen schmieren, MLG. grosen Saft aus Kräutern pressen.

113B4. DuDial. hutsekluts hodgepodge; intestines: hutse flabby gelatinous mass $+k l u t s$ small mass of firm objects.

113B5. MLG. kut, Prus. kitzing entrails, NHG. kitzing caviar. Cf. 70B2.

113B6. EDial. clytrie intestines, clyte mass of semiliquid material, clyter walking inelegantly over wet ground.

113B7. Swab. quantsch Eingeweide: quan(ken) einherwatscheln $+(q u a)$ tsch nasser, weicher Kot. Cf. 85A45.

113B8. Swiss chrotten toad's entrails; slimy mass of water plants, Fr. crotte excrement; mud.

113B9. Swiss mauw Eingeweide. Cf. 7H16.

113B10. Hess. rampen entrails of a cow, LG. ramp Sammlung verschiedener Sachen, rimpen falten, zusammenwerfen.

113B11. Thur., MLG. rampanien Eingeweide. Derived from the preceding.

113C1. DuDial. bel, bellen entrails of a herring, bellen hanging rags, WFlem. bel, belle bunch of flowers or seed pods hanging and swaying on a thin stalk, MDu. belle bell; bowl, NE. bell hollow body of cast metal to ring; name of several flowering plants, as bluebell, harebell.

113C2. NE. chitterling smaller intestines of beasts; frill, ruff or ornamental pleating, attrib. frilled, EDial. chitters small intestines of goose or sheep, NHG. kutteln phonetically remotely connected (NED.).

113C3. Swiss gehudel schlechte, abgetragene Kleider; zerfallene Baute; verwirrtes Garn; Eingeweide. Cf. 85B3.

113C4. NHG. lunze Eingeweide, Swiss lunzen faulenzend herumschlendern. Cf. 7K9.

113C5. DuDial. geslons entrails, EFris. gesluns Darmgeschlinge; schlotterig und nachlässig herunterhängendes Etwas, NHG. schlenzen nachlässig, müssig umhergehen.

113C6. MLG. paltuten Eingeweide: palt(e) Lappen, Stück+ (p) ute pudendum. Cf. $122 \mathrm{~K} 1$.

113D1. EDial. nudlens entrails, noodle strip of dough.

113D2. DuDial. snaar hog's intestines, Du. snaar cord, string. 
113E1. Fris. binnest entrails, OFris. binna within.

113E2. MLG. ingerif entrails, rif Leib. Compare Arnl. 139:09.

113F1. MDu. rop, roppe entrails, roppen rupfen, Swiss rupf was durch Rupfen gewonnen wird. Compare Arnl. 136:28.

113F2. NHG. gescheide Gedärm des Wildes. Cf. 146B1.

113F3. EDial. ush entrails, verb issue; clear, empty, cleanse.

113G1. Swiss burdi Eingeweide. Cf. 85C1.

113H1. Thur., Swab. bors, Bav. gebürsch entrails, Bav. Bürsch Börse, OHG. burissa Tasche, NHG. börse. Cf. 107A1, 129B2.

113J1. Fris. onganzen entrails. Cf. 129D4.

113K1. Swab. kranz, Swiss chranz wie nhd.; Gekröse, DuDial. krans Darmscheel.

113L1. DuDial. kurre entrails, Swab.kurren knurren. Cf.123M1.

113L2. EDial. rutt, ruttings entrails, ruttle rattle; gurgle.

113M1. Sax. schabanzen gekochte Eingeweide; Koder. From Wend. $z w a(n c)$ Labmagen + bansen paunch.

113M2. Sax. skalunze Gekröse: sch(abanze) (113M1)+ kal(daunen $)+(l)$ unze $(113 \mathrm{C} 4)$.

113N1. NE. singles entrails. Cf. $92 \mathrm{~N} 1$.

113P1. Swab. glen, glender Eingeweide.

113P2. Du. gelletje part of the entrails of a herring.

113P3. Swiss gragöl lautes Durcheinander, Unordnung; Eingeweide eines geschlachteten Schweines; Gerüstwerk.

113P4. EDial. medcalf calf's pluck: med(ley) mix + calf?

113P5. EDial. troistry entrails of a beast; offal, Icel. tros trash, Swed. trastyg trumpery (Jam.)

\section{ReCTUM}

The rectum is described (A) as a bunch, swelling; (B) as an end; (C) as a stem; (D) as a sack; (E) as a passage; (F) as various kinds of intestines.

114A1. Westf. dott grosser Darm, Dickdarm, Du. dot Knäuel, Büschel. Cf. 6E7, Wood, Mod. Phil., XI, 333.

114A2. Hess. günter Mastdarm des Schweines. Cf. 85A23.

114A3. EFris. knap-blase Schweinemastdarm, der von Kindern aufgeblasen und aufgestossen wird, damit er platzt: knap Schallwort+blase. 
114B1. DuDial. end end; rectum.

$114 \mathrm{C} 1$. Flem. steven rectum, MDu. stêvene stem, prow.

114D1. Thur. keutel starker Darm, NHG. keutel Fischnetz; Sack im Netz; sackförmiger Darm bei Tieren.

114E1. Swiss gang wie nhd.; Mastdarm.

114F1. Du. aarsdarm, NHG. arsdarm, dial. darsarm for arsdarm (Arnl. 136:09), Du. endeldarm, Steir. leibdarm, MLG., DuDial., EFris. pakdarm, NHG. packdarm (pack-intestine), weidedarm (Speise-darm) rectum.

\section{VISCERA}

The viscera are described (A) as a soft, confused mass; (B) as a lump, swelling; (C) as something hanging; (D) as something winding, entwining; (E) as something plucked out; (F) as something within; (G) as an accessory; $(\mathrm{H})$ as like the heart.

115A1. Schles. plauze Eingeweide, Lunge, NHG. idem, Swiss plauz weiche halbflüssige Masse, Sax. plauzen geräuschvoll, schwer auffallen. Or from Pol. pluca Lung (Wg.).

115A2. EDial. pummice heart, liver and lights of a lamb, same as pummey, pummice, pomace anything crushed to a pulp.

115A3. Ruhl. gerabbel essbare Eingeweide, Swiss räblete Krabbeln; Gepolter; Durcheinander, Hess. geräppel Menge kleiner und geringfügiger Sachen.

115A4. EDial. gorgetches calf's pluck, heart, liver and lights of an animal, gorgey shake, tremble, Sc. gorge noise made by the feet when the shoes are filled with water.

115B1. NHG. bäuschel lights, lungs, Bav. bäuschlein viscera of fish; compress for wounds, NHG. bausch ausgedehnter Wulst, Gebund Stroh.

115B2. Swiss grien Kies, Geröll von kleinen Steinen mit Sand; Lunge, Leber, Herz, Luftröhre und Kalbsbröschen, Als. grien grober Kies.

115B3. MLG. strutte viscera of animals, NHG. strotzen.

115C1. NE. hange, henge pluck or liver; lungs and heart, an early derivative of hang (NED.). Cf. 25D3.

115D1. NHG. gereb die oberen Eingeweide, OHG. reba, NHG. rebe (Wg.). 
115D2. NHG. geschlinge Schlund mit Lunge, Leber und Herz, welche daran hangen, Swab. geschlünge, geschlungel Gedärm, MHG. gestingen sich winden.

115E1. EDial. pluck heart, lungs; that which can be plucked. Cf. 113F1.

115F1. Fris. yntaest inmost part; viscera.

115F2. EDial. immeat edible viscera; food given to animals indoors: in +meat.

115G1. EDial. appurtenances heart, liver and lungs, NE. appendage; that which belongs to something.

115H1. DuDial. hartslag lung, liver and heart: heart+slag sort, kind.

\section{Appendix}

The appendix is described (A) as a sack; (B) as something hanging.

116A1. Bav. bodensack, Kärnt. sack, poud'nsack (poud'n Boden) Blinddarm.

116B1. Swiss gehenk appendix; Eingeweidenteile. Cf. 25D3.

\section{BLADDER}

The bladder is described (A) as a lump; (B) as a swimmer; (C) as the central part.

117A1. Bav. plunzen Urinblase. Cf. 9A2.

117A2. Fris. bonge, bongel bladder, bunge pig's bladder, NHG. bengel. Cf. 167B1.

117A3. Als. kneller Harnblase; Schwimmblase beim Fische. Cf. 26E3.

117B1. EDial. swimmer air bladder of a fish, NE. swim.

117C1. Neumark. zeele Fischblase, NHG. seele idem; das Innerste, Swiss sēl am Krebs der mittlere Teil des Schwanzes. Cf. 24E1.

\section{Bladder Duct}

The bladder duct is described (A) as a pipe; (B) as a spigot; (C) as a popper.

118A1. Swiss ror Harnröhre. Cf. 151B1. 
118B1. Lothr. buch-zappen (bauch-zapfen) Harnröhre.

118C1. Swab. kläpfer Klopfer an der Haustür; Harnröhre der Schweine, wird von Kindern aufgeblasen und zerknallt.

\section{Wомв}

The womb is described (A) as a swelling; (B) as such receptacles as oven, bag, box, cask; (C) that in which something rests, is housed; (D) as that which bears, carries; (E) as the procreating intestine; (F) as the womanly part; (G) as the mother.

119A1. Steir. brosch Uterus des Schweines, broschet dick, aufgedunsen, Swiss brösch strotzend von Milch. Cf. 81B4.

119A2. EDial. bliry exterior of a mare's uterus, NE. blear protrude, ME. bleren protrude the tongue.

119A3. Swiss blateren Luftblase im Wasser; blasige Anschwellung; Fruchtblase beim Vieh; Harnblase. Cf. 126A3.

119A4. EFris. rusel-darm Gebärmutter der Schweine, rusen aufschwellen. Koolman suggests rusel Unterleib.

119B1. Sax. backofen Backofen; Mutterleib: der backofen ist eingefallen sie ist entbunden.

119B2. EDial. bag womb of domestic animals. Cf. 81A1.

119B3. EDial. box uterus of cow or mare.

119B4. ME. butte butt, wine skin, cask; uterus, Fr. botte, boute cask, wine skin (NED.).

119B5. Lux. kalversak cow's womb, fillesak mare's womb. Cf. 85A46.

119C1. EDial. bed womb of an animal; bed.

119C2. DuDial. kalverhoes (calf-house) cow's womb.

119C3. Lux. luecht Eiertasche der Hühner, MLG. lucht das obere Stockwerk eines Hauses, auf dem Korn, etc., gelagert sind, KE. loft.

119D1. Bav. burd Bürde; Gebärmutter bei Kühen, Swiss burdi Tragsack; Gebärmutter bei Tieren. Cf. $85 \mathrm{C} 1$.

119D2. Swiss geburtglied Gebärmutter. Cf. 127Q1.

119D3. MLG. berwech uterus?: beren +wech Weg.

119D4. Als. trage Gebärmutter der Tiere, Bav. trag, tragsack idem, Swab. tracht idem. Cf. 129A5.

119E1. EDial. breeding-gut (breed procreate) womb of animals.

119F1. MLG. vrouwelicheit Gebärmutter. 
119G1. Du. moeder, EFris. mör, Oberh. mutter, Du. moederbed, moederschoot, Westf. moerwerk womb.

\section{Ovary}

The ovary is described (A) as the place where something is laid; (B) as a lump.

120A1. Fris. lecch bed; ovaries, Du. leg idem, leggen lay.

120B1. Swab. gromen ovaries. Cf. 126A10.

\section{Oviduct}

Fris. lechpiip (lay-pipe) oviduct. Cf. 120A1.

\section{Genitals, Pudenda}

The genitals are described (A) as that which begets; (B) as that which has intercourse; (C) as pudenda; (D) as something natural; (E) as a vessel; (F) as something hanging, loose; (G) as a bundle; (H) as something lively; (J) as both (sexes); (K) unclassified.

122A1. Fris. tyldeel, Du. teeldeel genitals, teellid penis: telen beget+deel part, lid Glied.

122B1. Swiss kumers Verkehr; pudenda, Fr. commerce Verkehr.

122C1. Steir. schande wie allg.; Schamteile. Compare NE. shame pudenda and Arnl. 140:16.

122D1. Swiss natur Naturtrieb, Geschlechtstrieb; semen; pudenda, EDial. nature pudenda of a mare.

122E1. Swiss gefäss Genitalien.

122F1. Swab. geschlamp pudenda; alles lumpig, schlaff, wirr herum-, herunter-hangende, NHG. schlampen schlotterig herabhängen.

122F2. Prus. kittel, LG. keddel Rock; genitals of male codfish.

122G1. Als. pack Pack, Bündel; male genitals.

122H1. NFris. lewent Leben; Genitalgegend, NHG. lebend.

122J1. Swiss bidi genitalia, namentl. membr. vir., bidi-beidi genitalia utriusque sexus, Grimm bidibidi hermaphroditus: bidi from beidi (Schw.). Compare zwitter, OHG. zwitarn and Wallonian boc et gate hermaphrodite.

122K1. MLG. pute private parts. Cf. 127B4. 


\section{Penis}

The penis is described (A) as that which dangles, sweeps, shakes, prods, etc.; (B) as a bat, club, pole, stick, etc.; (C) as a swelling, lump, knot, etc.; (D) as a cylindrical object, such as a finger, spout, cock, pipe, neck, root, etc.; (E) as something long and pliable, a cord, hose pipe, snout, snake, snail, etc.; (F) as a point; (G) as a borer; $(\mathrm{H})$ as that which urinates, spurts; $(\mathrm{J})$ as something small; $(\mathrm{K})$ as a gun; (L) as a bird; (M) as a growler; (N) as a peeper; (P) as a stinker; (Q) as a curl; (R) as manhood; (S) as something notched; (T) by euphemisms; (U) by proper names and appellatives.

123A1. Als. bumper, bimber, Swiss bimpel, bimper penis, Als. bimpel idem; Glockenschwengel, bimplen bimmeln, hin und her bewegen; coire.

123A2. Prus. pimmel penis des Knaben, bimmel der Baumelnde (Frisch.).

123A3. DuDial. stiersveē, -vede bull's penis; whip, formerly stiersvege, NHG. fegen: vede made over from veē, as if $d$ had fallen out.

123A4. EFris. fikker penis, fikken, NHG. ficken.

123A5. MLG. duve penis, duvete pudenda, EFris. duven stossen, drücken.

123A6. NHG. zagel Schwanz; penis, NE. tail. Compare NHG. schwanz penis (Arnl. 141:58).

123A7. Als. glunker etwas, was herabhängend baumelt; Beutel; penis mit Hoden, MHG. glunkern baumeln.

123A8. Swiss ginggel etwas herabhängendes und schaukelndes; penis, Als. ginkel freischwebender, hängender Körper; penis, ginkes penis, gungglen baumeln, schaukeln. Cf. 101B2, $126 \mathrm{~B} 2$.

123A9. Swiss gigel penis, gigerlen vor Kälte oder Angst zittern, NE. giggle. Cf. $127 \mathrm{~J} 1$.

123A10. Swiss gäuggis Geck; penis, gauggerlen sich hin und her bewegen, gaugglen lebhafte Bewegungen machen.

123A11. Swab. gus penis, Swiss guslen zappeln; mit den Fingern schnell nacheinander jemanden leicht stechen; schlagen.

123A12. Swiss gitzi Zicklein; junges, unruhiges, hüpfendes Mädchen; aus den Beinkleidern heraushangender Hemdzipfel; penis kleiner Knaben. To NHG. geiss? 
123A13. Westf. hämpelken penis, hampeln sich bewegen, EFris. gehampel Gezappel, hampelman jumping jack.

123A14. Hess. hiller penis, Thur. hille eilig, Grimm idem, hurtig.

123A15. Hess. hippel penis, Sax. hipel idem, hippeln hinken.

123A16. Als. kinkel Glockenschwengel; penis. Cf. 14A9.

123A17. Steir. klachel Glockenschwengel; penis. Cf. 126B4.

123A18. Swab. klunker Troddel; Glockenschwengel; pud. virilia, klunkeren baumelnd, schwankend herabhängen. Cf. 24B2.

123A19. Swiss quembel penis: LG. $k w(a b b e l)$ fetter, hervorstehender Körperteil+Swiss (b)ambel(en) baumeln.

123A20. EFris. lümmel dickes, schweres etwas; penis, NHG. lumm schlotterig, locker, weich.

123A21. Swiss nüttel penis?, notten sich hin und her bewegen. Cf. $85 \mathrm{~B} 7$.

123A22. Egerland wackl penis, MHG. wackeln, NHG. wackeln.

123A23. Als. wadel, wedel penis; Schwanz; herabhängendes Ende eines Seiles, NHG. wedel Büschartiges zu Hin- und Herbewegung.

123A24. WFlem., Flem. wiep mentula, Du. wippen sich schnell bewegen, MHG. wipfen springen.

123A25. Als. wusser penis, wusselen sich schnell bewegen, Kärnt. wusele penis, Lothr. wusselich lebhaft, zappelig.

123B1. Swiss bengel Bengel, Knebel; penis, MHG. Prügel, Swiss bingg ein Hinkender; penis, EFris. bingeln eine Glocke hin und her schwingen. Cf. 92A1.

123B2. EFris. bull-pitsk Ochsenziemer, pitsk Peitsche.

123B3. EFris. bull-stok Ochsenziemer, stok Stock.

123B4. NHG. deichsel penis, OHG. dīhsala Deichsel.

123B5. Steir. dreschel penis, OHG. driscil Dreschflegel.

123B6. Als. stecken penis. Cf. 69B13.

123B7. Fris. 'skacht penis of horses, NHG. schaft Stange als Stiel; penis of animals; Geburtsglied der Raubtiere, MHG. schaft shaft.

123B8. Swiss chelle mentula; Schlagholz. Cf. 74B7a.

123B9. EDial. knocker penis, knock coire.

123B10. Schles., Swab. heft penis. Cf. 9B13.

123C1. Swab. eichel wie nhd.; $p l$. glans penis. 
123C2. Swab. eisen wie nhd.; penis.

123C3. Swiss bonz penis. Cf. 127A5.

123C4. Swiss bir Birne; penis, MHG. bir pear. Cf. $92 \mathrm{~B} 2$.

123C5. Swiss bizoggel eine Art Nudeln; penis.

123C6. Swab., Swiss bolz penis arrectus, MDu. bout idem. Cf. $149 \mathrm{~A} 1$.

123C7. Tirol. buller penis. Cf. 127A10.

123C8. Swiss bummi penis; Prügel, Swab. bummel grosse Glocke, Swiss bummer kleiner, rundlicher Hund; kurze, dicke, dralle Person. Cf. 14A2, 90A16.

123C9. Swab. pommerlein kleines rundliches Gefäss; penis, Wald. pümel penis. Cf. 127A14.

123C10. Prus. pinsel penis, NHG. idem (of large game). Cf. $2 \mathrm{C} 1$.

123C11. DuDial. prul penis; worthless thing, Du. worthless thing, MLG. phallus ?, OFris. pralling testicle (Franck).

123C12. MDu. fluntse penis, Grimm finsen turgere, flans thick mouth.

123C13. Sax. schnippel penis, Prus. schnibbel idem. Cf. 7J8.

123C14. Bav. stumper kurzer, dicker Mensch; kurzes Glied; penis, stumpf stump; peris.

123C15. Swiss güggel penis; bewegl. Teil am Fasshahn; Tannzapfen, gugger rote Pustel; penis, Swab. gucker penis. Cf. 81B12.

123C16. Swiss hegel grobes Klappmesser; penis erectus; Grobian, Swab. gefährlicher Kerl.

123C17. Wald. huler penis, Grimm huller Pack, Ballen, hullern rollen.

123C18. Swiss chäfer Käfer; penis kleiner Knaben.

123C19. Als. kide penis. Cf. 9A8.

123C20. Swiss cholben, Swab. kolbe glans penis. Cf. 5A32.

123C21. Du., Fris. kul penis. Cf. 126A15.

123C22. Swiss müwel horse's penis. Cf. 7H16.

123C23. Steir. nudel was allg.; Erdapfel; penis, NHG. nudel.

123C24. Schles. nulle, nille, Thur., Sax. nille penis. Cf. 9 B19.

123D1. Sax. pfeife, Als. pfeif, Swiss pfïffe, DuDial. piep, Prus. pipe, Westf. pippel, LG. piephahn, pippig, Rap. pip penis. Cf. 9B3, $127 \mathrm{~B} 2$. 
123D2. Swab. finger (elfter), Als. finger Penis.

123D3. Prus. wurst Wurst; penis.

123D4. Als. wurzel penis. Cf. ESlang root idem.

123D5. Swab. daumen penis.

123D6. Bav. storch wie nhd.; penis.

123D7. Steir. zapfen penis.

123D8. Bav. zen, ochsen-zen penis, MHG. zein Gerte.

123D9. Swab. ganskragen long neck; penis, Swiss, Swab. gans Gans; Krug mit Ausgussschnabel; penis.

123D10. Swiss chängel penis. Cf. 168A1.

123D11. Swab. kerze penis.

123D13. DuDial. koker horse's penis, Du. cylindrical receptacle.

123D14. NHG. hahn, EFris. han, Prus. pishan penis.

123D15. EFris. lülle Röhre; penis, Du lul idem; sugar tit.

123D16. Als. riberle movable part of a cock; penis, NHG. reiben.

123D17. Swiss rueb Rübe; penis, NHG. rübe.

123D18. Swiss nagel Nagel; penis.

123E1. Westf. ossenpiederk Ochsenziemer: pie(sek) penis + (pi)d(ske)+Wald. (pis)erk (123E3).

123E2. Du. bulletouw Ochsenziemer, touw rope.

123E3. Du. pees tendon; bowstring; bull's penis; root, EFris. pese, pise, pesel, pisel, MDu. peseric, Du. pezerik, Prus. pesel, pesrick, Wald. piserk, NE. pizzle penis, MDu. pese thread, fiber, string. Cf. 88A2, 136A2.

123E4. Swiss riemen penis. Cf. 141B5.

123E5. Bav. schlauch penis. Cf. 9C1, 124A3, 127B8, $132 \mathrm{E} 2$.

123E6. Zwickauer schnekl penis. Cf. 5B4.

123E7. Schles. schnicke penis, schnickel idem; langer, hagerer Mensch, Grimm schnicker schlank, Swab. schnack langer, hagerer Mensch. Cf. 9C2.

123E8. Thur. schnuder penis, NHG. Schnupfen, Rotzfluss. Cf. 9L19.

123E9. Sax. schwippkedittrich penis: schwippke biegsam+dittrich Dietrich.

123E10. Bav. unker penis, unk Schlange (Schm.).

123E11. EDial. weasand gullet; penis, OE. wāsend gullet. Compare Arnl. 43:20. 
123E12. Als. wiechen Docht; penis. Cf. 92E1.

123F1. Swiss belli, Hess. bille penis, OHG. bil Spitzhacke, Schwert.

123F2. Swab. bubenspitz Knaben-penis; zugespitzte Nudel.

123F3. Westf. pinn Pflock; penis, EDial. pin penis.

123F4. Swab. dolch penis.

123F5. Swiss sabel, Lothr. sawel Säbel; penis.

123F6. Westf. stert Sterz; penis, NHG. sterz.

123F7. Bav. zipfel wie NHG; penis, Nös. zip, Moselfr. zippel, Thur. bullzipfel penis.

123F8. Swab. klammhaken eisener Haken; penis.

123G1. Swab. borer penis, OHG. borōn bohren.

123G2. Swab. näber, Swiss napper, Bav. nipper Bohrer; penis, NHG. naber, näber.

123H1. Swiss bisi, biseli penis, bisi machen pissen, probably from mach $b s-b s$ (Schw.).

123H2. Swiss brunzeli penis, Als., Swab. brunzer, Kärnt. prunzar idem, NHG. brunzen.

123H3. Lothr. pissert, Westf. piss penis. Cf. $127 \mathrm{H} 2$.

123H4. Hess. schnuller penis, NHG. schnullen saugen; harnen.

123H5. NHG. schudel penis, vulva, Kärnt. tschud'ln pissen; penis und vulva, Swiss schuderen pissen.

123H6. Als. seicher penis, Steir. seichzeug Scham, Kärnt. sācher penis, sāch'n mingere. Cf. $127 \mathrm{H} 5$.

123H7. Swiss sewer, seber penis, seweren, seberen pissen.

123H8. Als. sprutzer Wasserstrahl; penis, NHG. spritzen.

123J1. Swiss äderi-büderi penis: äderi dimin. of ader+büderli kleines und dickes oder im Wachstum zurückgebliebenes, unansehnliches Geschöpf; die kürzesten Stengel beim Hanf, buderen klein und unansehnlich bleiben.

123J2. Swiss bigger kleines Pferd; kleines Tier, Kind; kleiner Mensch; penis.

123J3. Als. bipperle kleiner Mensch; penis, bippele, bippi Küchlein, solang es noch die Flaumfedern hat; junges verzärteltes Mädchen, Als. bippelen kränkeln, bipperen coire, bippes penis; kleines dickes Kind, Oberh. bibbel, bibbes mentula. 
123J4. Steir. pepi penis, popper Puppe; kleines Kind; Eiterbeule (U-K.).

123J5. EFris. pink penis. Cf. 58A2.

123J6. Swab. närrchen penis; nicht normal gewachsene Pflanzen, Früchte.

123J7. Bav. zisel penis, Als. zisele Zeisig, kleiner Vogel, ziserlig klein.

123K1. Swab. pistol wie nhd.; penis; NHG. flinte idem; ESlang gun idem.

123L1. Schles. finke penis, Swab. fink idem. Cf. 9A6.

123L2. Swab. vogel wie nhd.; penis, voglen Vögel fangen; futuere, vogler qui saepe coit, Swiss vögi penis eines Knaben. Cf. $127 \mathrm{E} 1$.

123M1. Swab. kurrle penis, kurrlen kollern. Cf. $113 \mathrm{~L} 1$.

123N1. Swiss guggūs (guck aus) Versteckspiel; penis (etwas halb versteckt Hervorblickendes). Compare ESlang dodger idem. Or cf. $123 \mathrm{C} 15$.

123P1. Als. stinker wer stinkt; penis; Käse.

123Q1. EFris.krulle, DuDial.kruul penis, NHG.krulle. Cf. 5B5.

123R1. Swab. mannheit Männlichkeit; penis, EFris. manlikheid idem; Hoden, Bav. das manlich zaichen virilia.

123S1. Kärnt. lāchele penis, MHG. lāche Einschnitt in Holz oder Stein.

123T1. Swiss brueder Brüder; die beiden Hälften des Nusskerns; penis. Cf. $126 \mathrm{C} 1$.

123T2. MLG. dink, NHG. ding, DuDial. dink, ESlang thing penis. Cf. 127X2.

123T3. Prus. schlüssel penis, er hat den schlüssel verdreht er ist syphilitisch.

123T4. Sax. patengeschenk penis, bes. von kleinen Kindern.

123U1. Swiss götti Pate; penis.

123U2. Swiss heinrich (warmi heichle) saubere Gesellen; penis.

123U3. Als. krispines penis. From Krispi(nus) a saint.

123U4. DuDial. piet, NHG. peter, petermann, EDial. peter penis.

123U5. EDial. willy penis, willy-coat child's flannel petticoat.

123U6. Sax. zebedäus, Nos. zebedelli Zebedäus; penis. 
124. Prepuce

The prepuce is described as a sheath, covering.

124A1. MLG. schode Scheide, Gemächte. Cf. 127B7; Arnl. 146:02.

124A2. NE. sheath, Du. scheede prepuce of a horse. Cf. 127B6.

124A2a. DuDial. sluif prepuce of a horse, sloove idem, sloven hüllen.

124A3. NHG. schlauch prepuce of a horse. Cf. $123 \mathrm{E} 5$.

\section{Scrotum}

The scrotum is described (A) as a bag; (B) as a swelling, lump.

125A1. NHG. dial. beutel, EFris. bül, büdel scrotum, OHG. bütil Sack.

125A2. Swiss kulblasen scrotum, blasen Sack. Cf. 126A15.

125A3. DuDial. borze bag; scrotum, Du. beurs Börse. Cf. $113 \mathrm{H} 1$.

125A4. EDial. purse scrotum of an animal, NE. purse. Same as above.

125A5. Swab. hochbalg Hodensack, Hoden; beim Schlachtvieh die Umgebung der Hoden, balg Hülse, etc., MDu. culbalch scrotum. Cf. $126 \mathrm{~A} 15$.

125A7. Fris. ponge sack; scrotum, Goth. puggs Geldbeutel. Cf. $127 \mathrm{~B} 3$.

125A8. Thur., Sax. sack, Du. zak, balzak, MDu. culsac scrotum. Cf. 85A 46 .

125B1. Swab. blater Harnblase; scrotum, OHG. blätara Blase. Cf. 126A3.

125B3. Swiss bruech Schambein, -gegend; Hodensack des kastrierten Ochsen, Swab. bruchig dick; unbeholfen, OHG. bruoh Bruch, Hose. Compare Arnl. 112:11.

125B4. DuDial. klos scrotum, MHG. klōz Ball (Franck), Fris. kloatsek scrotum. Cf. $126 \mathrm{~A} 13$.

\section{Testicle}

The testicle is described (A) as a round object, swelling, lump; (B) as a dangle; (C) as brothers, companions (pair of testicles); (D) as something secret; (E) as that which rams; (F) unclassified. 
126A1. NHG. dial. ei Ei; Hode.

126A2. Lux. jik Schusser; Hode, EFris. jikkern jagen.

126A3. Steir. blatterling Hode des Ebers. Cf. 125B1, 119A3.

126A4. Lux. praumen Hoden, Westf. prume, NHG. pfraume plum.

126A5. EFris. pralling, DuDial. prallen, MLG. prallink, OFris. pralling testicle. Cf. 8A3.

126A6. NE. stones testicles.

126A7. Nös. gāl Hode, Swiss gallen, gāl wie nhd.; Niere im Mineralreiche; weiche Masse in den Fugen des Gesteins, Swab. galle harter Knollen am Gestein.

126A8. Swab. geschröt Hoden; Hodensack bei Tieren, NHG. schrot abgeschnittenes Stück; gegossenes Bleikorn. Cf. 141B3.

126A9. Swab. geld Geld; Hoden.

126A10. Swab. gromen Hoden, gromensack scrotum, Bav. gramel, grömlein Hode. Cf. 113B2. Lex.: Ital. grumo Klümpchen. Hyrtl: Lat. crumena lederne Tasche.

126A11. Rogasener hölko Hoden, Prus. hölke kleines Schiff, NE. hulk ship; bulky, unwieldy mass, EDial. large piece of food.

126A12. EDial. cobs testicles. Cf. 5A30.

126A13. MLG. klōt, Du. kloot, NHG. kloss, Westf. klōt, Neumärk. kleet, Fris. cloat, EFris. kloat, klote, Wald. klaut, Lux. klūt, Rogas. klööto Hode; Kugel, Ball, MHG. klōz geballte Masse, Ball, Kugel. Cf. 125B4, 53B1.

126A14. Swiss, Swab., Als. klucker kleine Kugel; Eidotter; Hode, MHG. klucker globus.

126A15. MLG. kūle Kolben; Geschwulst; Hode, MHG. kiule dickes, kugelkopfartiges Ende. Cf. 123C21.

126A16. Als. münze; Geld; Hodensack.

126A17. Als. mutschel Hoden beim Eber. Cf. 2 A36.

126A18. NE. nut testicle. Cf. $2 \mathrm{~A} 42$.

126A19. Egerland. nöian testiculi, NHG. nieren.

126B1. NHG. gagel testiculi. Cf. $101 \mathrm{~B} 1$.

126B2. Swiss gunggelen Hoden. Cf. 123A8.

126B3. Swab. behenk Hoden von Tieren. Cf. 25D3.

126B4. Bav. klächel Schwengel in einer Glocke; was niederhangend hin und her schwankt; scrotum; Testikel. Cf. $123 \mathrm{~A} 17$. 
126B5. Swab. kläpperes Hoden, klapperen klappern.

126B6. Als., Rap. schelle Klingel; pudenda; Hoden, NHG. schelle.

126C1. MLG. brodere Brüder; Hoden. Cf. 123T1.

126C2. MLG. geselle Genosse; Hoden.

126D1. Swab. heimen Hoden, heimlichkeit pudenda.

126E1. Bav. rammeln Hoden, Steir. ramler männl. Hase, NHG. rammel was rammt.

126F1. Swab. humen testicle.

\section{Pudendum Femininum}

The pudendum femininum is described (A) as a swelling, bump, bundle, etc.; (B) as a sheath, sack, pocket, etc.; (C) as a hole, hollow, crevice, split, notch, etc.; (D) as a vessel, container; (E) as a trap, clamp, catch; (F) as something hanging, a flap; (G) as something bushy, hairy; $(\mathrm{H})$ as that which urinates; $(\mathrm{J})$ as that which trembles, shakes; (K) as something rubbed; (L) as something convoluted, folded; (M) as something red; $(\mathrm{N})$ as a growler; $(\mathrm{P})$ as an instrument played upon; (Q) as a bearer; (R) as offal; (S) as a point; (T) as something in which to dip; (U) as a kitchen; (V) as an arch; (X) by euphemisms.

127A1. Oberh. bumbel pud. fem., Swab. bumpel idem; grosse Kartoffel, Sax. pumpe, Bav. pumpel vulva; dicke, plumpe Person.

127A2. Swiss bürzel vulva. Cf. 90A19.

$127 \mathrm{~A} 3 . \quad$ Als. butze cunnus. Cf. 5A10.

127A4. EDial. bullion pud. fem., NE. boss, stud, OFr. bouillon bubble, stud.

127A5. Oberh. bunze, bons pud. fem., Bav. punzen vulva; Pflaume; kurze dicke Sache, Thur. bunz pud. fem., Swab. idem; bauchiges Gefäss, Prus. punz vulva, Schles. bunzel, punzel idem, Swiss bünzli Pustel, Knötchen. Cf. 123C3.

127A6. Bav. bainz vulva. Cf. 127A5.

127A7. DuDial. barmt, barm, berm pud. fem. of animals, Du. barmte heap of earth or hay. Compare, further, Wood, Mod. Phil., $\mathrm{XI}, 326$.

127A8. Fris. bint, bunt cow's pud., Du. bond bundle, binden bind. 
127A9. Thur. bubbel pud. fem., Swab. poppel kugelförmiger, kleiner Körper, MDu. bobbel, bubbel Beule; Wasserblase, NE. bubble.

127A10. Hess. bulle vulva, Swab. bull idem; Katze; Stück von Pelz, bulle runder Zierrat, bolle runder Körper, Klumpen; Kugel; Beule, etc., OHG. bolla Fruchtbalg; Knoten des Flachses. Cf. $123 \mathrm{C} 7$.

127A11. Swab. bütte cunnus, MHG. bütte Bütte.

127A12. Fris. print pud. fem. of cows and sheep, EFris. printjen zusammennähen und flicken, prinen stecken und heften.

127A13. Du. prut inspissated matter; curds; pud. fem., adj. thickened, pruttelig knorrig.

127A14. Prus. pum cunnus, pummel etwas Umwickeltes, Bepummeltes, EFris. kurzes, dickes, rundlich-volles Etwas. Cf. $123 \mathrm{C} 9$.

127A15. Prus. vagotze cunnus: vag(ina $)+(f)$ otze. Cf. $92 \mathrm{C} 4$.

127A16. Bav. feigen wie nhd.; feminal, Ital. la fica der Daumen bei geschlossener Faust zw. Zeige- und Mittelfinger durchgesteckt, imago vulvae seu coitus (Schm.).

127A17. Du. venusberg, venusheuvel (hübel), maagdenberg mons veneris.

127A18. Westf. fissenülle pud. fem.: vise(l), MHG. visel penis +nülle 123C24.

127A19. Thur., Sax. hummel cunnus, EFris. hummel Erhöhung, Grimm hummel Rindvieh ohne Hörner.

127A20. Swiss nest, Swab. hühnernest cunnus. Cf. 5A42.

127A21. Swab. hutzel cunnus; Tannenzapfen, hutzlen zusammenschrumpfen. Cf. 81B15.

127A21a. Swab. kappe, Swiss pelzchappe Mütze; cunnus.

127A22. Bav. kuschel pud. fem. Cf. 7J2.

127A23. Swiss chrūsel Krug mit weitem Bauch; pud. fem; dickes, fettes Weib, MHG. krūse Krug, etc.

127A24. Thur. deise, Steir. duserl Dose, pud. fem. of a cow, Swab. dus Dose. Cf. 90A24.

127A25. Thur. trumpe pud. fem. of a cow. Cf. 9A17.

127A26. Swab. maus, Swiss mūs cunnus. Cf. 51D4.

127A27. Thur. meise, Westf., Wald. möse pud. fem., Swab. meise idem; kleines Kind, Schles. mēse pud. fem., urspr. meise 
Tragkorb, Prus. mese, möse Mütze; vulva EFris. mesken cunnus, MHG. meise Tragkorb.

127A28. Swab. mock pud. fem., grosses dickes Stück, MHG. mocke Klumpen.

127A29. NHG. dial. mutz vulva. Cf. 7H20.

127A30. Swiss nuss vulva Cf. $2 \mathrm{~A} 42$.

127A31. Steir. rumpumpel pud. fem.: rum(pel) altes Weib+ pumpel (127A1). Wood, JEGPh., XI, 322.

127B1. NHG. dial. büchse Büchse; cunnus. Cf. 90A13.

127B2. Als. pfeif cunnus. Cf. 123D1.

127B3. Prus. ponke cunnus Cf. 125A7.

127B4. EFris. püt, püt, MLG. pute, Schles. pützel, Kärnt. putz'n pud. fem., EFris. püt Sack, Beutel.

127B5. NHG. dial. tasche Tasche; pud. fem. Cf. 7F3.

127B6. Swiss scheid, Du. scheede vagina. Cf. 7F6.

127B7. Sax. schote vulva. Cf. 124A1.

127B8. Bav. schlauch pud. fem. Cf. 123E5.

127B9. Prus. kott cunnus; Hülle, NE. cod bag, husk; scrotum, OE. codd bag, husk. Compare Arnl. 142:02.

127C1. Bav. schlipp Einschnitt; cunnus, NHG. schlippe enger Weg.

127C2. Sax. schlitz vulva, NE. slit.

127C3. Als. schnatte Schnitt; pud. fem., schnatt Schnitt.

127C4. DuDial. spleet crack; pud. fem., NE. split.

127C5. Fris., Du. gat cunnus. Cf. 91A3.

127C6. Swab. gefüge cunnus, NHG. crack, joint.

127C7. Thur., Hess. kaute Grube, Rinne; pud. fem.

127C8. Du. kut pud. fem., NHG. dial. kutte, ME. cutte idem, Als. kutt Grube, Loch. Cf. 91A7.

127C9. Swiss chlimse längl. Vertiefung, Ritze; cunnus, Steir. klumse idem, MHG. Spalte.

127C10. Du., Fris. klink split; pud. fem. of a cow, WFlem. klinke split, seam; region between anus and pud.

127C11. Swiss, Swab. loch, Westf. mīgloch cunnus, mīgen Harn lassen.

127C12. Steir. lücke as NHG.; vagina.

127C13. Steir. rabuschel pud. fem., rabusch Kerbholz. 
127C14. Prus. machēle, machīle cunnus. Cf. 91A9.

127D1. NHG. dial. pfanne Pfanne; vulva. Cf. 90E8.

127D2. Steir. pritsche receptacle for tools under a wagon; pud. fem., Swab. idem. Cf. 7H5.

127D3. Swiss chlachlen irdenes Gefäss; pud. fem., Swab. kachel idem, MHG. kachele irdenes Geschirr.

127D4. Bav. schumpel, Kärnt. tschumper feminal, Czech žumpa Pumpe, Wasserbehältnis (Schm.).

127D5. Thur. schnumpel pud. fem.: schn(uder) penis (123E8)+ (sch)umpel 127D4.

127D6. Schles. schachtel wie hd.; pud. fem.

127D7. Als., Lothr. schatull vag. fem., NHG. schatulle.

127D8. Swab. lade cunnus. Cf. 7F8.

127D9. Steir. rührkübel Butterfass; membr. fem.

127D10. Bav. wannen vulva, wanne Behälter, Badewanne.

127E1. Schles. vogeleisen feminale. Cf. $123 \mathrm{~L} 2$ and ESlang mousetrap cunnus.

127E2. Swab. gaffeisen cunnus, gaffen gape. Cf. 7G2.

127E3. Steir. heinzelbank Schnitzbank mit einem grossen Kloben zum Festhalten der Holzstücke; vagina.

127E4. NHG. kloben feminale, MHG. klobe gespaltenes Holz zum Klemmen.

127E5. Gron. knibbelkist cunnus, kist Kiste. Cf. 73B1.

127E6. Thur. schnalle pud. fem., NHG. Schliessgeräte.

127E7. EDial. snatch pud. fem., verb catch hold of.

127F1. Swab. bampel vulva. Cf. 8C1.

127F2. Swiss bletz Lappen; vulva, strauwbletz Matte aus Strohgeflecht; pud. mul., MHG. bletz Lappen. Cf. $105 \mathrm{C1}$.

127F3. Swiss chlēp, chlepp cunnus, NHG. klappe.

127F4. DuDial.trut, WFlem.trutte pud.fem., truttelen loiter, trifle.

127F5. Swab. zusel, Kärnt. tschuschele pud. fem., Tirol. zussl herabhängender Lappen; lumpig gekleidetes Weib.

127F6. Schles. lunze feminale. Cf. $7 \mathrm{~K} 9$.

127G1. Swab. pelz, Swiss belz Pelz; cunnus. Cf. 5C1.

127G2. Oberh. puschel pud. fem., NHG. büschel.

127G3. Steir. puschkawaukerl Feminal: pusch Busch +ka(wanzel) zottiger Hund, Pudel+wauckerl kleines Ding. 
127G4. Swiss busi, büsi etwas Flockiges, Wolliges, sich weich Anfühlendes; Flocke Wolle, Haar; vulva, Als. bus cunnus, Swab. buse idem; Katze, junges Gänschen, Swiss buslen vulva; Katze; Samenkopf der Disteln. Cf. 62B1.

127G5. Swiss bausen vulva. Cf. 5C3.

127G6. Swab. katze Tier; Wollgras; weiche, pelzartige Blüte; cunnus.

127G7. Swab. maunze Katze; cunnus. Cf. 35A5.

127G8. Swiss zizi-bauderen cunnus: zizi katze+bauder Kater. 127H1. Swab. blatt-feicht pud. fem. des Wildes: NHG. blatt pud. fem. (Arnl. 143:39)+Bav. feicht feucht, feuchten mingere. Or for feigenblatt?

127H2. Fris. pisser cunnus. Cf. $123 \mathrm{H} 3$.

127H3. Prus. quinzillge cunnus; Weib, das bei jeder Gelegenheit weint: quin(telieren) weinen (Swed. kvintilera trill)+Sax. zille hochaufgeschossenes Frauenzimmer, das kindisch, träg, ungeschickt ist.

127H4. Hess. quintipse vulva. Cf. preceding.

127H5. Steir. seiche vagina. Cf. $123 \mathrm{H} 6$.

127J1. Bav. giggewiz'n vulva, gigel inneres Beben. Cf. $123 \mathrm{A9}$.

127J2. NE. twat pud. fem., Bav. zwazeln zappeln, MHG. zwatzler penis. Compare Arnl. 141:64.

127K1. Kärnt. fumb'l vulva, fumb'ln coire, Steir., Nös. fummel vulva, Obersachs. fummeln schnell hin und herreiben, Swab. coire; blank reiben.

127K2. Swab. fuse cunnus, Grimm fuseln an etwas hin und her bewegen.

127L1. Lothr. hans, Bav. ansen Geburtsteile der Kuh, Fr. anse handle of a basket, pot; bay, cove; curve in a nerve or intestine where it doubles back upon itself, Lat. ansa Henkel, Bogen (Foll.).

127L2. Swab. krummei pud. fem., NHG. krumm.

127L3. Steir. rungunkel altes runzeliges, übellauniges Weib; cunnus: rungg(en) brummen, murren $+(r)$ unkel, MHG. runke Runzel. Compare Wood, JEGPh., XI, $322 \mathrm{f}$.

127L4. Steir. schneck pud. fem. Cf. 5B4.

127M1. DuDial. roode, roo pud. fem., Kil. roode omasum, Du. rood, roo red. 
127N1. DuDial. preute cunnus, Du. preutelen grumble. Cf. 90G4, $123 \mathrm{M} 1$.

127P1. Swab. klavier cunnus. Cf. 53D1.

127Q1. Steir. geburt vagina, Swab. geburtglieder genitalia. Cf. 119D2.

127R1. DuDial. barrel something bad; offal; cow's pud., Du. offal.

127S1. EDial. pen sow's pud. Cf. $5 \mathrm{H} 3$.

127T1. NHG. tunke Flüssigkeit zum Tunken; cunnus; NHG. tunken tauchen.

127U1. Swiss chuchi, Swab. kuche, ESlang kitchen pud. fem. Cf. $104 \mathrm{C} 1$.

127V1. Als. schluss wie nhd.; head of a door, window; weibl. Scham im mediz. Sinne, Bav. schloss idem, NHG. schliessen.

127X1. Swiss blueme Blume; Jungfrauschaft; pud. mul.

127X2. Steir. dingerl vagina. Cf. 123T2.

127X3. Swab. kirchweih Kirchweih; cunnus; menses.

127X4. Swab. hundsfuchs pud. of female dog, euphem. for hundsfotz (Foll.).

127X5. Swab. grusel Gans; Wasseralf; pud. fem.

127X6. Swab. girlitz Hänfling; weibl. Glied.

\section{HYMEN}

The hymen is described as the maiden membrane: Du. maagdenvlies hymen, vlies skin, membrane.

\section{Afterbirth}

The afterbirth is described (A) as a birth, afterbirth; (B) as a sack; (C) as a cleanser, sweeper; (D) as offal, filth; (E) as stuff, goods; (F) as the mother-liver; $(\mathrm{G})$ as a bed; (K) as a tuft; (L) as the lightener.

129A1. MLG. echterste (aftermost) Nachgeburt?

129A2. NHG. afterbürde, NE. afterburden, -birth, NHG. nachgeburt, Fris. neigeboarte afterbirth, MHG. bürde Getragenes.

129A3. Swiss burt, geburt, burdi, Steir. bürtel, Bav. bürdlein afterbirth, OHG. giburt Geburt, Gebähren. 
129A4. Swiss nachlast Nachgeburt, NHG. last.

129A5. Als. tragete Traglast, Nachgeburt der Kuh. Cf. 119D4.

129B1. Fris. pōk afterbirth of cows. Cf. 85A41.

129B2. Bav. faselbursch Nachgeburt. Cf. $113 \mathrm{H} 1$.

129C1. Thur. feh Nachgeburt, fegen fegen; die Nachgeburt werfen, Swab. fege Nachgeburt der Kuh, NHG. fegen.

129C2. Lothr. rän, Als. rein, reinte, reinet Nachgeburt, Swiss reini Reinsein; Nachgeburt der Kuh, NHG. rein.

129C3. Swiss richten Nachgeburt, Swab. drichte (d'richte), verricht idem, richten in Ordnung bringen.

129C4. EDial. cleansing placenta of cows and sheep.

129C5. Bav. säubere, Swiss süberi Sauberkeit; Nachgeburt, Bav. sich säubern Nachgeburt ablegen.

129C6. Bav. schoen Schönheit; Nachgeburt, DuDial. schoone idem, NHG. schön.

129D1. DuDial. verwerp afterbirth of cows, NHG. verwerfen.

129D2. EFris. fülsel Fäulnis; Nachgeburt, fül unrein.

129D3. Oberh. los Nachgeburt, NHG. idem; Excremente beim Wild, losen den Kot gehen lassen (Wg.).

129D4. DuDial., Fris. ongans afterbirth: on neg. +gans sound. Cf. $113 \mathrm{~J} 1$.

129E1. EFris. tüg Zeug, Gerät; Nachgeburt, NHG. zeug.

129E2. EFris. godje monatl. Reinigung; Nachgeburt, god Gut; Zeug; Wäsche; Nachgeburt.

129F1. Du. moederlever afterbirth, lever liver.

129G1. Flem. bed afterbirth of mare or cow, Lothr. bett afterbirth, OHG. betti Bett, Beet.

129K1. Oberh. büschlein Nachgeburt; Strauchwerk, diesem Vergleichbares.

129L1. EFris. ligt, Du. licht afterbirth, EFris. ligt, licht, leicht. Cf. 99B1. Compare (C) and Franck, ${ }^{2}$ p. 383.

\section{Caul}

The caul is described (A) as a cap or net; (B) as a smear.

130A1. NE. caul any small net; peritoneum, pericardium; membrane which encompasses the head of a child when born, OFr. cale cap. 
130A2. EDial. how coif, headdress; nightcap; caul, OE. hüfe covering for the head (Wr.).

130A3. Fris., EFris. helm helmet; caul, Swiss Nachgeburt der Pferde.

130B1. EDial. smear caul; roe of fish; an ointment, etc., verb rub sheep with tar.

\section{Membrane}

The membrane is described as something veined: NHG. reiss fette Netzhaut, Swiss gereisselet geadert, OHG. reiss linea, nota.

\section{SkIN}

The skin is described (A) as a covering, such as hull, bark, leather, etc.; (B) as a swelling; (C) as something bare; (D) as something thin and broad, like a leaf; (E) as a pipe; (F) as a strip; (G) by a transferred meaning.

132A1. Sax. bast Haut der inneren Handfläche, Fris. bark, covering; corpus, properly skin, DuDial., MHG. bast Rinde.

132A2. NE. buff bare skin; leather. Fr. buffle buffalo (NED.).

132A3. EFris. bulster Polster; Hülse, Decke; Haut, Balg, Du., MLG. bolster Hülse; Nussschale.

132A4. Swab. decke Bettdecke; Haut.

132A5. MDu. cortse pelt, fur; cover, Lat. cortex bark; shell.

132A6. Swiss rinde verdickte Hautschicht.

132A7. DuDial. schilt skin, hide, MDu. scilt shield.

132A8. MDu. schubbe hide, fleece, scale, NHG. schuppe.

132B1. Flem. hot skin. Cf. 85A27.

132B2. Cologne huck Haut, NHG. hucke Traglast.

132C1. Fris. bloat, bleat sheared sheepskin, bleat nudus.

132D1. Steir. blatt ausgezogene Tierbalg, NHG. blatt.

132E1. Swiss chänneli frisch abgezogenes Fell eines Zickleins; ausgeweidetes Schlachtvieh, MHG. kanel Röhre.

132E2. Bav. schlauch Haut der Schlange. Cf. 123E5.

132F1. MLG. queden Bauchfell der Eichhörnchen, EFris. kwedder Randbesatz, Streifen Zeugs, der auf die Kante eines Kleidungsstücks gesetzt wird.

132G1. Steir. klaue Fell vom Fusse der Tiere; Fell über'haupt. 


\section{EPIDERMis}

The epidermis is described as the pain skin: DuDial. smartvel, EFris. smartfel epidermis, smart Schmerz.

\section{Scale, Shell}

The scale of a fish is described (A) as something split off; (B) the shell of insects is described as an inclosure; (C) the shell of crabs is described as a hull; (D) unclassified.

134A1. Du. schelfer, schilfer splinter; fish scale, schelfe shell, hull, splinter; scale, $M L G$. schelver piece split off.

134A2. Cologne schölp Fischschuppe; Schinne, Als. schülp Schuppen auf dem Kopf, Ditm. schulp hull of rapeseed. Cf. 134A1.

134B1. Swiss ranft Kruste; hornartige Körperhülle von Insekten, MHG. ranft einfassender Rand. Cf. 7L1.

134C1. Als. schafele Schale, Hülle; Kruste der Krebse, schife Schote von Hülsenfrüchten, Bav. schefen idem.

134D1. MLG. vlome, LG. flomen Fischschuppen; Nierenfett.

\section{PORE}

The pore is described as a hole: NHG. schweissloch pore; Du. zweetgat pore.

\section{TENDON}

The tendon is described (A) as a hair, thread, fiber, cord; (B) as a leader; (C) as to the tongue ligament as that which stretches; a stitch; (D) unclassified.

136A1. EDial. paxwax strong tendon in the neck of animals; gristle in meat. An older form was faxwax: OE. feax hair+weaxan grow (NED.).

136A2. Du. pees tendon, achillespees tendon of Achilles. Cf. $123 \mathrm{E} 3$.

136A3. DuDial. fas tendon, MDu. vase, OHG. faso Faser. Cf. $150 \mathrm{D} 1$.

136A4. EDial. ficfac sinew, tendon, fixfax idem. Cf. 136A1.

136A5. NHG. flechse, flachsader tendon, OHG. flahs flax (Wg.).

136A6. Du. geelhaar (yellow hair) sinews in meat, langhaar idem. 
136A7. EDial. gyves fetters; sinews of the legs, NE. fetter.

136A8. NE. cord tendon. Cf. 138A1.

136A9. NE. hamstring the great tendon at the back of the hock of the hind leg of quadrupeds, dial. string idem. Cf. 138A2.

136B1. EDial. leader one who leads; tendon; sinew; artery.

$136 \mathrm{C} 1$. Flem. spanader (stretch vein) linguae vinculum.

136C2. EDial. tack stitch; tongue ligament, verb stitch.

136D1. Wald. haise starke Flechse am Bein.

138. Muscle

The muscle is described (A) as a cord, string; (B) as a soft mass; (C) as a pointed object; (D) as to a certain one as the patience muscle.

138A1. EDial. cord muscle. Cf. 136A8.

138A2. EDial. strings muscles of the uterus of a cow; certain tendons and muscles of the human body. Cf. 136A9.

138A3. Swiss band muscles of the uterus.

138B1. Swiss mauwen Fleisch ohne Knochen; Muskel. Cf. $7 \mathrm{H} 16$.

138C1. Du. spier grass shoot; muscle, MDu. grass shoot; pointed stick.

138D1. Du. geduldspier (patience muscle) the muscle which raises the shoulder blade. Cf. $138 \mathrm{C} 1$.

139. BLOOD

The blood is described (A) as soup; (B) as fat.

139A1. Prus. suppe (rote) Blut.

139B1. Bav. faist Blut des Hirsches, faisten bluten, MHG. veize Fett, NHG. feist fat, Bav. hirschfaisste time when the stag was fattest.

\section{Flesh, FAT}

The flesh or fat is described (A) as something soft, flabby; (B) as something flat; (C) as a filler; (D) as that which is grasped; (E) as a flower; (F) as a stocking; (G) by a transferred meaning.

140A1. Als. brustleffel Rindfleisch von der Brust, OHG. laffan lecken, schlürfen. 
140A2. Lux., Westf. wabbel herunterhängendes Fleisch; hervordrängendes Fett, wabbelen herunterhängen.

140A3. Cologne geschluddersch Fleisch, schluddern schütteln, schwingen, NHG. schlottern.

140A4. Sax. geschlumpe lockeres Fleisch, MHG. slump schlumpig.

140A5. Du. kwab flabby flesh. Cf. 15A1.

140A6. Prus. labber weiches, schwammiges Fleisch, LG. labberig schlaff, Du. labber soft, slack.

140A7. Prus. ladder weiches Fleisch, LG. ladde filzartige Decke über einem Sumpfe. Cf. 140A9.

140A8. Als. lämpen loses, hängendes Fleisch, Bav. Fetzen; herabhängende Fetthaut, lampen schlaff herabhängen. Cf. 8C5, 14A16, 81C7.

140A9. Prus., Lothr. luder Fleisch; Aas, NHG. Fleischfülle an Saugtieren; Aas, EFris. dickes, weiches Fett, weiches fettes Fleisch; Aas, MHG. luoder Lockspeise; Schlemmerei, Russ. láta Flick, Lappen. Compare, further, Wood, MLN, XXIX, 71.

140B1. DuDial. vla, vlage layer of fat on the inner side of the skin, NE. flag piece cut out of the sward; flat slab.

140B2. Neumärk. fliize Fettschicht um die Nieren; Platte aus Stein, NHG. fliese Stein-, Tonplatte.

140C1. Steir. fülz Bauchfett der Schweine, Swiss fülli Füllsel, Swab. fülle Füllung.

140D1. Swiss, Bav. griff Stellen am Körper des Rindviehs, an denen man durch Greifen den Grad der Fettigkeit prüft; der wulstige Brustteil, Fett zw. den Hinterkeulen, Swiss schwanzgriff Sitzbeingegend beim Rindvieh. Cf. 90S1.

140E1. Sax. blume Nierenfett der Schweine.

140F1. Cologne bogstrump Fleisch am Vorderschenkel des Rindviehs: OHG. buog oberes Gelenk des Schenkels+NHG. strumpf. 140G1. Flem. lies inner fat, fat of the belly. Probably transferred from lies groin. Compare Arnl. 119:01.

\section{Flesh (to eat)}

The flesh as meat is described (A) as something hard, bony, cloddy, gristly, stringy, leathery; (B) as something cut, split off, 
a strip or band; (C) as something slaughtered; (D) as food; (E) as something short; (F) as a kid.

141A1. Cologne geknoosch knorpeliges Fleisch. Cf. 150B5.

141A2. Cologne geknöchs Kleinfleisch mit vielen Knochen, NHG. knochen.

141A3. Swiss geragel etwas Beiniges; hageres Geschöpf; Fleisch mit vielen Knochen; Schlechtes, hartes Obst, MHG. rac steif, straff.

141A4. EDial. clod clodbeef, coarse part of the neck.

141A5. Sax. sohle Rindfleisch. Compare NE. soleleather tough meat.

141A6. Thur. zattern, Sax. zatter ungeniessbare Fleischteile, Sehnen, zattrig faserig, OHG. zata herabhängende Haare, Faden, Wolle.

141A7. Du. nagel-hout smoked beef. Cf. 68A7.

141A8. Swiss ränggel zähes, schlechtes Kuhfleisch, ringgel, runngis altes, zähes Fleisch, runngleten mangelhaft entwickelte Früchte.

141B1. Cologne kluft Fleischteil im hintern Gelenkteile, LG. wie nhd.; dickes Fleisch in der Bauchhöle, Fleischballen in der Keule, buten- oberer, binnenkluft unterer Teil der Keule. Compare Cologne bennespalt (binnen-) Batzenfleisch des Hinterbeines.

141B2. Bav. schalen Schale; Fleisch an den Hüften, am Schweif; Fleischteil gewonnen durch Spalten des Knöpfls, DuDial. schilstuk meat cut from the front shank, EFris. schil Hülse, etc.

141B3. Nös. schroden grosses Stück Fleisch, schrot abgeschnittenes Stück, schroten abschneiden. Cf. $126 \mathrm{~A} 8$.

141B4. Swiss binden Streifen zum Binden; aufgehängte Fleischriemen, Bundnerfleisch.

141B5. Swiss riemen Streifen Fleisches, NHG. riemen. Cf. $123 \mathrm{E} 4$.

141C1. Swab. kafel Fleisch, kafelen schlachten.

141C2. Swiss metzg Schlachthaus; Fleisch, MHG. metzje Fleischbank, Schlachthaus.

141D1. EFris. met bestes Fleisch vom Schwein, MLG. reines Schweinefleisch, NE. meat food; flesh of animals, OE. mete, Goth. mats food, etc. 
141E1. Cologne gekööz fettes Rindfleisch zw. den Rippen, kööze kürzen.

141F1. Swab. kitze junge Ziege; Fleisch, NHG. kitze.

142. BACON

Bacon is described as something flat: Als. blächel sehr fetter Speck, geräucherter Speck, MHG. blach flach.

\section{QUICK}

The quick is described as a kernel: Flem. kornel flesh under the nails, WFlem. kernel, kornel idem; also of horns and hoofs, NE. kernel.

\section{HORN}

The horns are described (A) as forks; (B) as pincers; (C) as crooks; (D) as projections; (E) as ear ornaments; (F) unclassified.

145A1. Swab. gabel Hörner des Rindviehs. Cf. 50D2.

145B1. Swiss chluppen Geräte zum Festklemmen; einwärts gebogene Hörner eines Rinds; Krebsschere, Swab. kluppen klemmen.

145C1. EDial. crooker cow's horn, NE. crook.

145D1. Tirol. grul etwas weit Hervorstehendes; grosse Hörner. Cf. 26G1.

145E1. Fris. ear-izer (ear irons) cow's horns.

145F1. DuDial. heure horn.

\section{ANTLER}

The antler is described (A) as a point; (B) as something branching; (C) as a projection; (D) as an ornament; (E) as a crown.

146A1. Oberh. spiss deer's horn of two prongs, MHG. spiz spit.

146A2. Bav. gezinde stag's horns, zint Zacke, gezindelt zackig.

146B1. Bav. geschaid Ende am Hirschgeweih; Scheidelinie, OHG. geskeite Teilung. Cf. 113F2.

146C1. EDial. brow overhanging brushwood; antler, also browantler, browpoint, NE. brow prominent ridge over the eye.

146D1. Bav. gezierde Geweih, NHG. zierde.

146E1. Swab. krone Geweih des Rehbocks. Cf. 79D1. 


\section{Antenna}

The antenna is described (A) as a stick; (B) as a horn.

147A1. Swiss chnebel Bartfaden der Fische, NHG. knebel.

147B1. Du. hoorn, voelhoorn antenna.

\section{STING}

The sting is described as something sharp, pointed.

148A1. DuDial. ang bee sting; beard of grain, ON. angi Spitze.

148A2. Swab. dorn bee sting, OHG. dorn Dorn.

148A3. Swab. nadel bee sting, NHG. nadel.

148A4. NE. sting sharp pointed organ of certain insects, OE. stingan thrust, stab, pierce. Cf. 92K1.

148A5. DuDial. straal sting; fang of serpents. Cf. 79G1.

\section{JoINT}

The joint is described (A) as to the ball, as a bolt, knob, nut, etc.; (B) as to the socket, as a hollow vessel; (C) as a link; (D) as a hinge; (E) as that which turns; (F) unclassified.

149A1. Swab., Swiss bolz bolt; joint. Cf. 123C6.

149A2. NHG. dial. kegel Gelenkkopf, NHG. kegel.

149A3. DuDial. keute ball of a joint; hip bone; knucklebone, keuter instrument to poke with, pipe cleaner, etc.

149A4. Swiss chnode Gelenkknoten, -kopf, Swab. knote Knöchel am Fuss, Fingergelenk, NHG. knoten. Cf. 50A13, 53B3, 74B11, $77 \mathrm{~A} 2$.

149A5. MDu. cnoop knob, joint, NHG. knauf Knopf, Knoten. Cf. $42 \mathrm{~A} 8$.

149A6. Schles. kobel Gelenkknochen der Schafe, Swab. Berghöhe, Erdscholle.

149A7. DuDial. noot ball of a joint, Du. noot nut.

149A8. EDial. whirl flywheel; round knob of wood; hip joint.

149A9. Swiss würtenschnopf Kopf des Hüftgelenks, NHG. wirtel verticellum; whorl; Knopf.

149B1. Du. pan socket of a joint, Swab. pfanne, Als. gelenkpfanne idem, NHG. pfanne. Cf. 90E8.

149B2. Fris. potsje socket of a joint, Du., NE. pot. 
149B3. Flem. hot socket of a joint. Cf. 85A27.

149C1. EDial. link joint, NE. link. Cf. 5B9.

149D1. DuDial. her joint of the jaw, MDu. harre, herre hinge.

149E1. Fris. wringe joint, verb wring, NE. wring.

149E2. Du. gewricht joint. From *gehwerchte from *gehwerfte, NE. whirl (Franck). Cf. 149A8.

149F1. NFris. lass Gelenk, Glied.

\section{Cartilage}

The cartilage is described (A) as that which crunches, crackles; (B) as a knot, lump; (C) as something soft, easily crushed; (D) as a fiber.

150A1. EFris. gnister Knorpel, MLG. gnistern knirschen.

150A2. Du. knarsbeen, MDu. cnorsel, Fris. knarsbien, DuDial. gnors, gnorzebot cartilage, Du. knarsen crunch.

150A3. DuDial. knirselbonk cartilage, knirsen crunch. Cf. 150A2.

150A4. DuDial. knosper, knasper cartilage, knosperen, knaspen crackle.

150A5. Lothr., Als. krachel, Du. kraakbeen, Fris. kreakbien cartilage, NHG. krachen.

150B1. Du. knorfbeen, knorf cartilage, knorf large person. Compare Arnl. 159:05.

150B2. MDu. cnorre knot; knob; cartilage, DuDial. knarre, knorrebot idem, NHG. knorren.

150B3. Prus. knurspel Knorpel. From *knur(pel) (knorpel)+ (kro) spel cartilage.

150B4. MLG. knoster Knorpel, LG. knūst, knuist, knusten, knoost rundlicher Auswuchs; hartes Ende vom Brot.

150B5. MDu. geknoos, Cologne knoosch cartilage, NHG. knösel Knirps; zwerghaft verkrüppeltes Ding. Cf. $141 \mathrm{~A} 1$.

150B6. Swiss chnotzer Knorpel, MHG., NHG. knotze Knorren.

150C1. Du. weekbeen cartilage: week weich+been.

150C2. EDial. crish, crush cartilage; soft bones of young animals, easily crushed by the teeth, verb crush.

150D1. DuDial. vas cartilage; heels. Cf. 136A3. 
151. BONE

A bone is described (A) as a lump, chunk; (B) as a pipe; (C) unclassified.

151A1. Du. bot, MDu. bot, but bone, Du. bot stumpf, LG. butt idem, kurz und dick, Du. bot Auswuchs an Bäumen, EFris. botten schwellen. Cf. 90A10.

151A2. LG. hunke abgenagter Knochen, MLG. hunke-ben Kernkammer des Obstes; Schinken, von welchem das Fleisch fast ganz heruntergeschnitten ist, Flem. hunke hump, NE. hunk large lump, piece. Compare Wood, MLN, March, 1914.

151A3. EFris. klufe Klumpen; Knochen, Bein, MLG. kluve gespaltenes Holz, um die Hände zu fesseln, kluven split.

151A4. Bav. ranken Fussknochen, Knochen; Kalbsfuss; mageres Vieh, NHG. grosses Stück Brot, Fleisch.

151B1. Als. rohr, Swiss ror, rören Rohr; Röhrenknochen, NHG. rohr. Cf. 8A8, 43A1, 118A1.

151C1. Swab. fuchs Knochen mit Mark.

151C2. EFris. blainen whalebone.

\section{Frontal Bone}

The frontal bone is described (A) as a lid; (B) as a kernel.

152A1. Du. oogklep (eye clap) horse's frontal bone, klep lid.

152B1. Du. beenpit (bone pit) bony knob where cows' horns grow, pit pit, fruit stone.

\section{Malar Bone}

The malar is described as a yoke bone: Du. jukbeen malar, juk yoke.

\section{JAWBONE}

Part of the jawbone is described as a drum: Du. trommelbeen (drum bone) bone fastening the lower jaw to the temple (birds).

\section{EтhmoId Bone}

The ethmoid bone is described (A) as a sieve; (B) as a saddle. 155A1. Du. zeefbeen (sieve bone) ethmoid bone, zeef seive.

155B1. Du. zadelbeen (saddle bone) part of the ethmoid. 
156. INCUS

The incus is described as an anvil: NE. anvil small bone of the ear.

\section{Hyoid Bone}

The hyoid is described as a gaping bone: Du. gaapbeen (gape bone) hyoid bone, gapen gape.

\section{SPINE}

The spine is described (A) as the backbone; (B) as a spring; (C) as a swelling, ridge.

158A1. NE. backbone spine.

158B1. EDial. sprent sudden start or spring; vigorous effort; elastic force of anything; spring at the back of a pocketknife; spine.

158C1. Oberh. hochrück Rückgrat.

158D1. Kärnt. schwal, schwol Rückgrat; Bergrücken, Grimm schwall schwellende, geschwellte Wassermenge; körperliche Dehnung.

\section{Spinal Cord}

The spinal cord is described (A) as a cord; (B) as a power sinew. 159A1. Flem. strang spinal cord, NHG. strang.

159B1. MLG. walt-sene Rückgrat: walt Gewalt+sene Sehne.

\section{Vertebra}

The vertebra is described (A) as that which turns; (B) as something rounding; $(\mathrm{C})$ as a kind of man.

160A1. Du. draaier turner; second vertebra, draaien drehen.

160A2. EDial. naabar, naavar upper vertebra of a sheep's neck; nape of the neck, Shet. naver augur.

160A3. Du. wervel roller; vertebra, MLG. werveltop vorderes Ende des Rückgrats, wervelen turn.

160A4. MDu. wordel vertebra, Lat. vertere.

160B1. Swiss chralle Gegenstände, welche Korallen ähnlich sind; knolliges Gras; Wirbel des Rückgrats.

160C1. Swiss jud jude; Wirbelknochen des Schweines, der die Form eines sitzenden Männchens haben soll, Lothr., Thur. idem. 


\section{Breastbone}

The breastbone is described (A) as a fork; (B) from its use as a slinger, as a springer, etc.; (C) as like that part of eyeglasses fitting on the nose; (D) as the bone which fulfils desire, contributes to joy.

161A1. Swiss gablen Gabel; Schlüsselbein an Vögeln.

161A2. Du. vorkbeen (fork bone) breastbone.

161A3. MDu. spriet two-tined fork; forked bone, NHG. forked pole.

161A4. Fris. bok, fugelbok (fowl buck) breastbone of a bird. The breastbone of birds bears a similarity in shape to a goat's head with horns.

161B1. Als., Swab. springer Brustbein der Vögel, zu einem aufschnellenden Spielzeug verwendet. Cf. 169B1.

161B2. Sax. hüpfauf, hippel, Swab. hopfhans Brustknochen der Vögel (used as 161B1), NHG. hüpfen.

161C1. Siebsächs. augenspigel Augenglas; Hühnerbrustbein, das scherzw. als Augenglas auf die Nase gesetzt wird.

161C2. Du. bril eyeglasses; breastbone of birds, NHG. brille.

161D1. NE. wishbone, wishingbone breastbone of fowls, over which wishes are made. Or compare Wood, Mod. Phil., II, 476.

161D2. NE. merry-thought breastbone of fowls. Cf. preceding.

\section{Chavicle}

The clavicle is described (A) as a ring; (B) as a pipe; (C) as a convenience for hanging; (D) as a keybone.

162A1. Als. halsrinken clavicle, rinken ring.

162B1. ME. canel-bon collarbone, canel canal, channel. Cf. $41 \mathrm{~A} 5$.

162C1. Swizz löffelhenki Schlüsselbein bes. magerer Frauenpersonen oder Kinder, an das man, da es vorsteht, Kochlöffel aufhängen könnte, henki hanger.

162D1. NHG. schlüsselbein, Du. sleutelbeen clavicle: formed in imitation of Lat. clavicula.

\section{Long Bones of the Arm}

An arm bone is described (A) as a spindle, spoke; (B) as a pipe. 
163A1. Swab. armspindel Armknochen, MHG. Spindel Spindel; dünner Knochen.

163A2. NHG. speiche spoke; bone of the forearm.

163B1. Du. pijp pipe; pijpen in den arm hollow bones of the arm, ellepijp thickest bone in the lower arm.

\section{Carpus}

The carpus is described as a pea-bone: Du. erwtbeentje carpus, erwt pea.

\section{Phalanges}

The phalanges are described as members: DuDial. lit bone of toe or finger, OHG. lid Glied.

\section{KNUCKLEBONE}

The knucklebone is described (A) as a knot, knob, etc.; (B) as a notch.

166A1. EDial. knot knuckle. Cf. 2 A29.

166A2. Du., Fris. bikkel knucklebone, marble, EFris. also knee, elbow, MHG. bickel Würfel.

166A3. EDial. dib knucklebone of a sheep used as a plaything; small water pebble.

166A4. Prus. knebel Knöchel, NHG. knebel.

166A5. Steir. knüpel Knöchel, NHG. knüppel.

166A6. Thur. knorren Knöchel, NHG. knorren.

166B1. EDial. nick indentation; notch; groove; knuckles.

\section{HIP BoNE}

The hip bone is described (A) as a triple bone; (B) as a bump, knob, etc.; (C) as a hook; (D) as a hasp; (E) as a point; (F) as to the flat part, as a blade; $(\mathrm{G})$ as the intestine bone; $(\mathrm{H})$ as something heavy.

167A1. NHG. dreibein os coxae, von den drei Teilen davon.

167B1. MLG. bunk Knochen, namentlich die hervorragenden Hüft- und Beinknochen grosser Tiere, MDu. bonke, bunke, Du. bonk bone, Westf. bonke, EFris. bunke, Fris. bonke idem; foot, Prus. bunken die starken Knochen, namentlich magerer lebender Tiere, 
welche stark hervorragen, Pommern bunken-knaken grosser Knochen an der Hinterkeule, Norw. bunka kleiner Haufe, Beule. Cf. 74B1.

167B2. EDial. huggin hip, hip bone, hock bone, esp. of horse or cow. Probably from huckbone idem, huck hip; haunch, Ger. *huk- to be bent (NED.), NE. huckle hip; bunch, projection like the hip.

167B3. EDial. hubbon hip, hip bone, EFris. hubbel Unebenheit, Höcker. Or from hip +bone. Cf. 167B2.

167B4. NHG. eisbein, MLG. iss-bēn, MDu. ijs-, ise-been, NE. ice-bone os sacrum. Compare Arnl. 117:06.

167B5. Fris. hysbeen hip bone of a pig. From the preceding with $h$ from heup hip.

167C1. EDial. hook bend, turn; hip, hookers bended knees. Cf. 78A2.

167D1. MDu. hespe hinge; joint, hip joint; ham, Du. hesp ham. Cf. 9Dd1.

167E1. EDial. pin pin; hip, hip bone. Cf. 69B7.

167F1. Swab. blatt Hüfte. Cf. 46A6.

167G1. Du. darmbeen flat part of the hip bone.

167H1. Westf. hiewe Hüfte, hiewig heavy?

168. FEMUR

The femur is described (A) as a pipe; (B) as a club.

168A1. MDu. cangel Schenkel, MHG. kengel Röhre; Federkiel. Cf. $123 \mathrm{D} 10$.

168B1. Oberh. schlegel Schenkelknochen der Tiere; Hinterkeule, NHǴ. schlegel.

\section{Shank Bone}

The shank bone is described (A) as a pipe; (B) as a spring.

169A1. NE. cannon bone extending from the knee or hock to the foot, Fr. canon tube, pipe.

169B1. Du. hazesprong (hare spring) bone of the hind leg of a hare, sprong Sprung. Cf. 161B1.

\section{Splint Bone}

The splint bone is described as a pointed object: Du. griffelbeen splint bone of a horse, griffel graver, slate pencil. 


\section{TaIL Bone}

The tail bone is described as a point: Du. kam comb; pointed projection on the last joint of the tail of a bird, NHG. kamm.

\section{Fishbone}

A fishbone is described (A) as a prickle, sharp point; (B) as chaffy stuff.

172A1. Kurhess. habe Granne; Fischgräte, Hess. hebe Granne, Dorn.

172A2. Prus. staks Stich, $p l$. die feinen Gräten der Fische, auch kleine Fische nennt man stakse, stakswerk.

172B1. MDu. leme fishbone; husks, straw, Du. leem woody part of flax cast away.

172B2. Lux. sprei Fischgräte, NHG. spreu Abfall von Getreidehülsen, OHG. spriu idem.

\section{Periosteum}

The periosteum is described as a bone skin: Du. beenvlies (bone membrane) periosteum.

\section{RIB}

The rib is described (A) as something flat; (B) as something curved; (C) as to the short rib, as a point; something thin.

174A1. Swab. feder Rippe des Hirsches. Cf. 92H1.

174B1. Du. krap pig's rib, NHG. krapfen Krümmung zum Fassen und Einhängen, Haken.

174C1. EDial. pens short ribs. Cf. $5 \mathrm{H} 3$.

174C2. Neumärk. dünnlinge short ribs: dünn+ling. From this is probably derived Neumärk. didelinge short ribs.

\section{SKeLEToN}

The skeleton is described (A) as a collection of bones; (B) as something interwoven, such as a basket, framework; (C) as something shriveled; (D) as a cart.

175A1. NHG. gerippe Knochengerüst, collective to rippe.

175A2. Oberh. geriffel Gerippe: *riffel (Du. rif skeleton) with prefix from gerippe. 
175A3. Swab. gestilet Skelett: sti(let) dagger, suggested by skelett, with prefix from gerippe.

175A4. EFris. rif Gerippe; cadaver, MLG. rif, ref Leib, Leichnam, Du. rif skeleton, NHG. reff altes Weib. To rib? Compare Arnl. 103:14. Cf. 175A2.

175B1. EDial. frame skeleton; structure of fitted parts.

175B2. Swiss gerigel Fachwerk, Gerippe, rigel Querholz.

175B3. Swiss hutte Rückenkorb; Rauchfang aus Rutengeflecht; Gerippe von Geflügel. Cf. 85A27.

175B4. Swiss chräze Gerippe eines Tieres; Vorderteil am gekochten Geflügel, chrāze der hintere, beste Teil von gekochten Geflügel. Cf. 89A11.

175B5. Als. krett skeleton of fowl; Korb. Cf. 90A43.

175B6. EDial. rames skeleton, carcass; framework of anything, OFr. rame, raime branches; faggots. (Wr.).

175B7. Swiss ram Ramen; Gestell von Brettern; Gerippe, Cologne gerämsch Gerippe, MHG. rame Stütze, Gestell.

175C1. Swiss gringel Gerippe; ausgehungertes Geschöpf; etwas Kleines, Verwachsenes, Zugeschrumpftes, wie z.B. Apfel, Birne, granggel verkümmertes, geringfügiges, schwächliches, verwachsenes Ding; mageres Tier; ungestaltes, mageres Stück Fleisch. Cf. skeleton.

175E1. Swiss charre cart; wheelbarrow; skeleton of fowl.

\section{QUARTER}

A quarter of an animal is described as a thick object: MDu., Du. bout quarter of a slaughtered animal; thigh of fowl. Cf. 149A1. 



\section{INDEX}

(The figures refer to section numbers, not pages)

Abdomen, 85

Abomasum, 106

Adam's apple, 42

Afterbirth, 129

Ankle, 77

Antenna, 147

Antler, 146

Anus, 91

Appendix, 116

Arm, 47

Arm bone, 163

Back, 89

Backbone, 158

Bacon, 142

Beak, 12

Beard, 35

Belly, 85

Bend of the knee, 73

Bladder, 117

Blood, 139

Body, 1

Bone, 151

Bosom, 82

Brain, 39

Breast, 81

Breastbone, 161

Buttocks, 90

Calf, 70

Cannon, 169

Carpus, 164

Cartilage, 150

Caul, 130

Cheek, 15

Chin, 13

Clavicle, 162

Claw, 60

Crest, 34

Crop, 101

Crotch, 65

Crown, 32
Dewlap, 14

Diaphragm, 112

Ear, 25

Eardrum, 25F

Ear lobe, $25 \mathrm{H}$

Ear tubes, 25G

Elbow, 48

Enamel, 20

Entrails, 113

Epiglottis, 42C

Epidermis, 133

Ethmoid, 155

Eye, 26

Eyebrow, 30

Eyelash, 29

Eyelid, 26K

Eye socket, 28

Face, 6

Fat, 140

Feather, 162

Femur, 168

Fin, 63

Finger, 53

Fishbone, 172

Flank, 93

Flesh, 140

Flesh (to eat), 141-42

Flipper, 64

Fontanelle, 38

Foot, 74

Forefinger, 55

Forehead, 4

Frontal bone, 152

Gall, 102

Genitals, 122-28

Gill, 16

Gizzard, 193

Gland, 109

Glottis, 42B 
Groin, 66

Gullet, 44

Gum, 18

Gut, 113

Hair, 5

Hand, 50

Head, 2

Head (bald), 3

Heart, 96

Heart valve, 98

Heel, 78

Hip, 68

Hip bone, 167

Hoof, 79

Horn, 145-46

Hymen, 128

Hyoid, 157

Incus, 156

Instep, 76

Intestine, 113

Jaw, 17

Jawbone, 154

Joint, 149

Kidney, 100

Knee, 71

Kneecap, 72

Knee joint, 73

Kneepan, 72

Knuckle, 166

Lap, 95

Larynx, 42

Leg, 69

Ligament, 136

Limb, 67

Lip, 8

Little finger, 58

Loin, 94

Lung, 99

Malar, 153

Mane, 36

Membrane, 131

Membr. vir., 123
Middle finger, 56

Mouth, 7

Muscle, 138

Nail, 59

Navel, 87

Neck, 40

Nipple, 81

Nose, 9

Nostril, 10

Omasum, 105

Ovary, 120

Oviduct, 121

Palate, 21

Palm, 51

Pancreas, 110

Parting of the hair, 33

Paw, 52

Penis, 123

Pericardium, 97

Periosteum, 173

Peritoneum, 107

Phalanges, 165

Pit of the stomach, 86

Pleura, 108

Podex, 90

Pore, 135

Prepuce, 124

Pudenda, 122-28

Pud. mul., 127-28

Pupil, 27

Quarter, 176

Quick, 144

Rectum, 114

Retina, 26H

Rib, 174

Rump, 90

Scale, 134

Scapula, 46

Scrotum, 125

Shoulder, 45

Shoulder blade, 46

Side, 93 
Sinew, 136

Skeleton, 175

Skin, 132-33

Skull, 37

Snout, 11

Sole, 75

Spine, 158

Spinal cord, 159

Splint, 170

Sting, 148

Stomach, 104

Tail, 92

Tail bone, 171

Teat, 81

Temple, 31

Tendon, 136

Tentacles, 147

Testicle, 126

Thigh, 68

Thorax, 83

Throat, 41

Thumb, 54
Thymus, 111

Toe, 80

Tongue, 22

Tonsil, 23

Tooth, 19

Trunk, 1

Udder, 81

Umbilical cord, 88

Urethra, 118

Uterus, 119

Uvula, 24

Vertebra, 160

Viscera, 115

Waist, 84

Wing, 61

Windpipe, 43

Withers, 40

Womb, 119

Wool, 5

Wrist, 49 


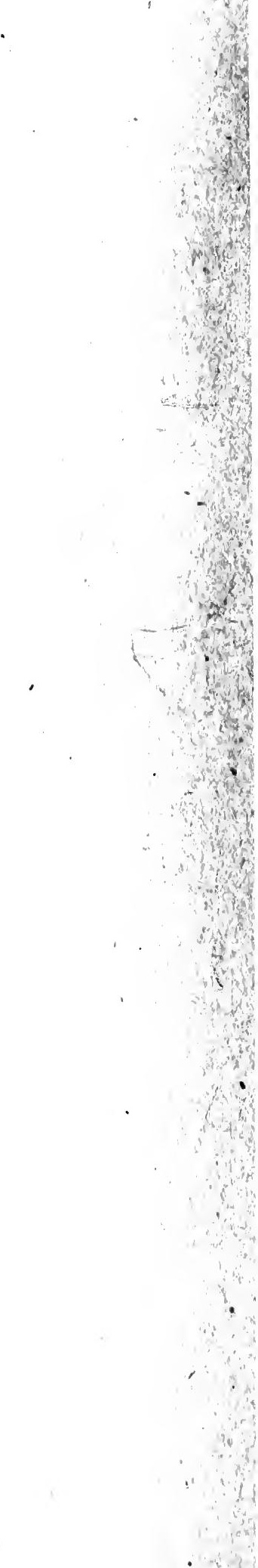



